\title{
Development of Novel Analytical and Therapeutic Applications of Aptamer Technology
}

\author{
by \\ Michael A. Beking, B.Sc. \\ A thesis submitted to the Faculty of Graduate and Postdoctoral Affairs \\ in partial fulfillment of the requirements for the degree of
}

Master of Science

in

Chemistry

Carleton University

Ottawa, Ontario

(C) 2012

Michael A. Beking 
Library and Archives

Canada

Published Heritage

Branch

395 Wellington Street

Ottawa ON K1A ON4

Canada
Bibliothèque et

Archives Canada

Direction du

Patrimoine de l'édition

395 , rue Wellington

Ottawa ON K1A ON4

Canada
Your file Votre référence

ISBN: 978-0-494-87833-0

Our file Notre référence

ISBN: $978-0-494-87833-0$

\section{NOTICE:}

The author has granted a nonexclusive license allowing Library and Archives Canada to reproduce, publish, archive, preserve, conserve, communicate to the public by telecommunication or on the Internet, loan, distrbute and sell theses worldwide, for commercial or noncommercial purposes, in microform, paper, electronic and/or any other formats.

The author retains copyright ownership and moral rights in this thesis. Neither the thesis nor substantial extracts from it may be printed or otherwise reproduced without the author's permission.
AVIS:

L'auteur a accordé une licence non exclusive permettant à la Bibliothèque et Archives Canada de reproduire, publier, archiver, sauvegarder, conserver, transmettre au public par télécommunication ou par l'Internet, prêter, distribuer et vendre des thèses partout dans le monde, à des fins commerciales ou autres, sur support microforme, papier, électronique et/ou autres formats.

L'auteur conserve la propriété du droit d'auteur et des droits moraux qui protege cette thèse. $\mathrm{Ni}$ la thèse ni des extraits substantiels de celle-ci ne doivent être imprimés ou autrement reproduits sans son autorisation.
In compliance with the Canadian Privacy Act some supporting forms may have been removed from this thesis.

While these forms may be included in the document page count, their removal does not represent any loss of content from the thesis.
Conformément à la loi canadienne sur la protection de la vie privée, quelques formulaires secondaires ont été enlevés de cette thèse.

Bien que ces formulaires aient inclus dans la pagination, il n'y aura aucun contenu manquant. 


\section{Abstract}

Aptamers are single stranded oligonucleotides which bind with high affinity and selectivity to their targets, and possess several properties that have generated excitement for their use across a number of disciplines. As the library of known aptamer sequences grows, so too does the potential for novel diagnostic, analytical, and therapeutic applications. Presently, two novel applications of aptamer technology will be described.

Electrochemical biosensors employing aptamers as the recognition element are widely reported, often presenting excellent sensitivity and selectivity. The current work demonstrated how the superimposition of an electrocatalytic cycle could further improve the sensitivity of such a biosensor without altering aptamer affinity. Specifically, a methylene blue aptamer tag was used for electrocatalytic reduction of hexacyanoferrate(III), resulting in a 7-fold improvement in sensitivity versus a sensor construct where the signal resulted from methylene blue reduction alone. Synthesis of the requisite probe-modified aptamer, and optimization of parameters such as the operating buffer, substrate concentration, and target incubation time will be detailed.

Magnetic Resonance Imaging (MRI) is the preeminent imaging modality in modern medicine, producing highly resolved images often with the aid of paramagnetic gadolinium chelates serving as contrast agents (MRI-CAs). Aptamer-gadolinium conjugates have been proposed in undergraduate work as a new class of actively targeted MRI-CAs for therapeutic application. In the present work, improvements to the synthesis of these conjugates, their magnetic characterization, and a re-envisioned route to the formation of di-substituted conjugates will be described. 


\section{Acknowledgements}

Despite the (attempted) humourous approach taken to some of the following acknowledgements, all parties mentioned and un-mentioned should be aware that they have my sincerest thanks for their support throughout my program or in my life before and after.

First of all, thank you to my family for their unending love and support, regardless of the quality of my life decisions or the number of self-deprecating comments that I make. Thank you for feeding me on Sundays, I can't imagine that I would have survived if left to make my own eating decisions seven days a week. As with my undergraduate thesis, this report is dedicated to the memory of my late father, who continues to inspire me every day. I am also most grateful to the Department of Chemistry, OGS, and NSERC for the financial support which made my studies possible.

An outrageously large thank you to Dr. Maria DeRosa for her support and encouragement throughout both degree programs. This project could not have been completed without her guidance and contagious energy. I would otherwise probably be lying in a metaphorical gutter, destitute and confused, with nary a banana to eat. Thank you as well to Dr. Bob Burk for excellent advice throughout my time at Carleton, and inciting my defection from engineering in first year.

While this list is by no means comprehensive, thank you for the following: To G.M. - for love and understanding. Despite the detrimental impact on my ability to communicate effectively, to T.F. for his pioneering observations on the first-year-noise. A.G. - for forming the most level-headed third of the $330 \mathrm{SC}$ survival committee. A.C. - 
for conversations over unnecessarily large bowls of pho. M.M. - for the pep-talks, and providing an example of general awesomeness. A.F. - for creating the head-impact zone for the times when I felt that electrochemistry had defeated me. E.B. - for allowing me to pretend to be her significant other at Costco. E.M. - for making sure I was well acquainted with the snowbanks on campus. To R.W. and Y.S., thank you for the wellintentioned motivational insults.

Following in the tradition of D.B., thank you to Dean Martin, Five Finger Death Punch, Times of Grace, and Flight of the Conchords for providing the soundtrack to the thesis writing process. 


\section{Table of Contents}

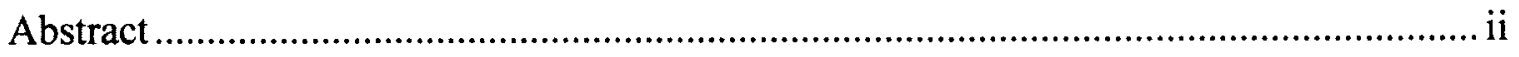

Acknowledgements.................................................................................................ii

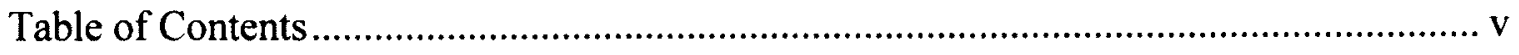

List of Tables ............................................................................................................... vii

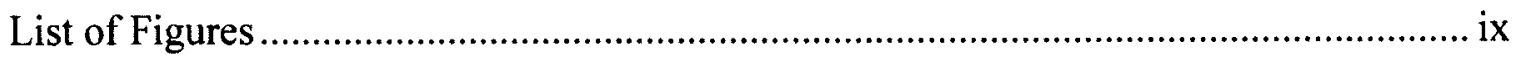

List of Abbreviations ........................................................................................... xiv

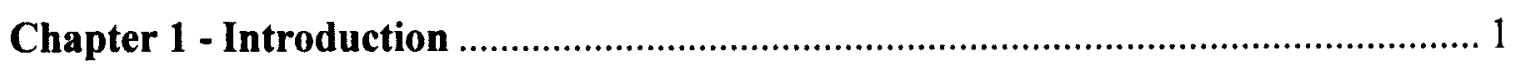

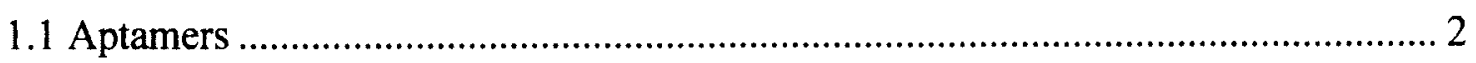

1.2 Comparison of aptamers to related affinity probes ……............................................ 4

1.3 Applications of aptamers...................................................................................... 7

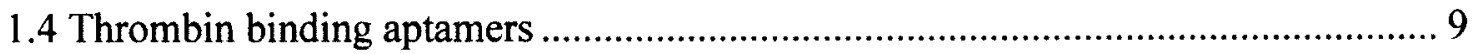

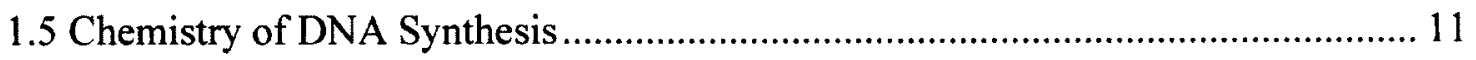

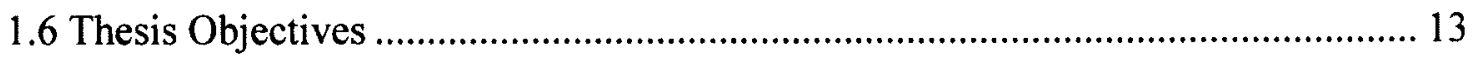

Chapter 2 - The enhancement of electrochemical aptasensors via probe electrocatalysis........................................................................................................ 14

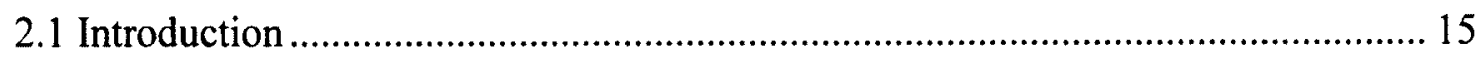

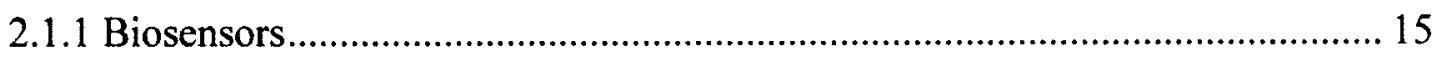

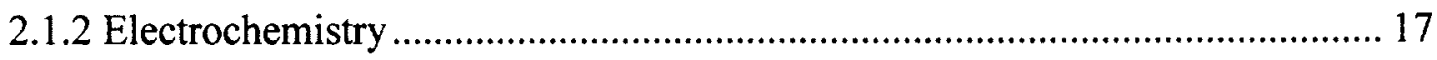

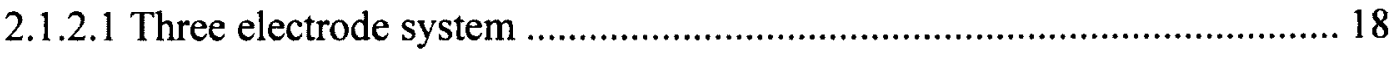

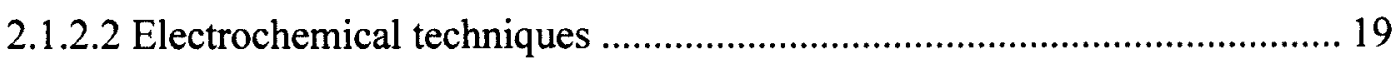

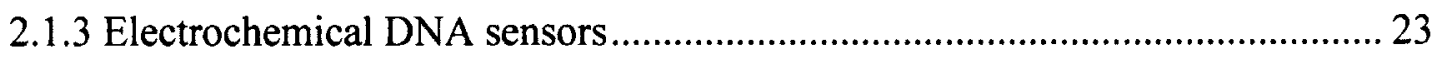

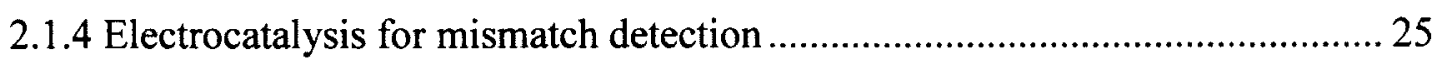

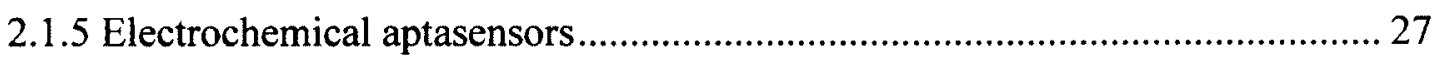

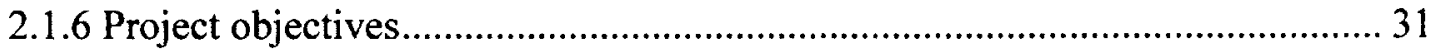

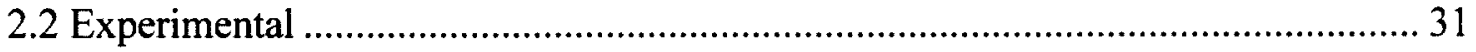


2.2.2 Synthesis of methylene blue-modified thrombin aptamer (MB-DNA) ........... 32

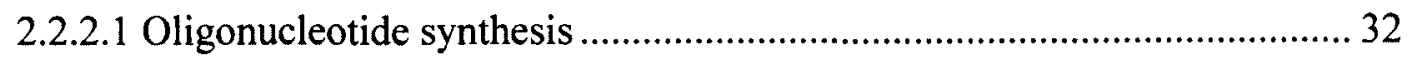

2.2.2.2 Conjugation of methylene blue to DNA .......................................... 33

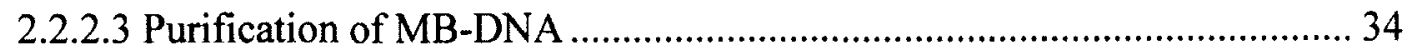

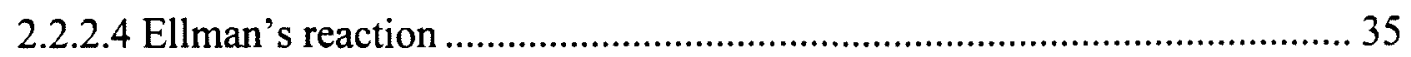

2.2.2.5 Other approaches to MB-DNA synthesis and purification ...................... 36

2.2.3 Preparation of MB-DNA modified electrodes ........................................... 39

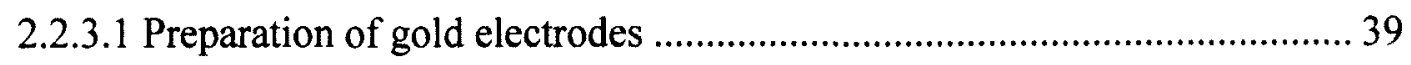

2.2.3.2 Deposition of MB-DNA and SH-DNA on gold electrodes ..................... 41

2.2.3.3 Treatment with 6-mercapto-1-hexanol ........................................ 41

2.2.4 Electrochemical characterization of modified electrodes........................... 42

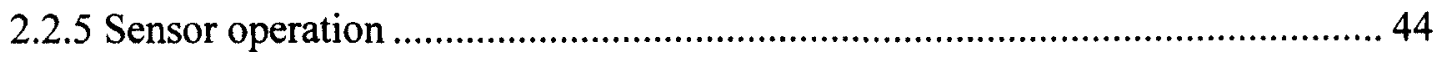

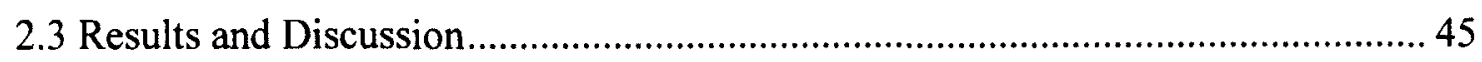

2.3.1 Preparation of methylene blue-labeled thrombin aptamer (MB-DNA)........... 45

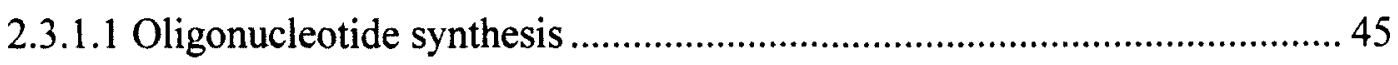

2.3.1.2 Synthesis and purification of MB-DNA …...................................... 46

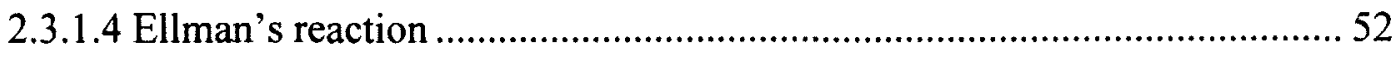

2.3.1.5 Other approaches to MB-DNA synthesis and purification ...................... 54

2.3.2 Preparation and electrochemical characterization of modified electrodes ....... 56

2.3.3 Optimization of thrombin incubation time and the role of cations.................. 59

2.3.4 Determination of conditions for electrocatalysis...................................6 62

2.3.5 Electrocatalytically enhanced aptasensor for thrombin .............................. 72

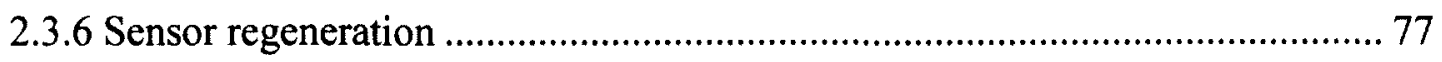

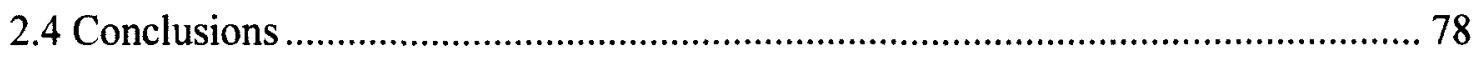




\section{Chapter 3 - Development of aptamer-gadolinium conjugates as magnetic resonance}

imaging contrast agents

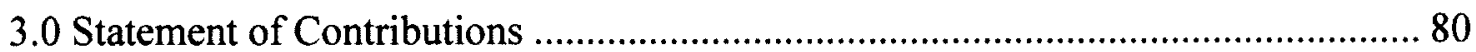

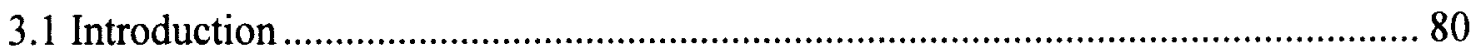

3.1.1 Magnetic resonance imaging contrast agents (MRI-CAs) …............................ 80

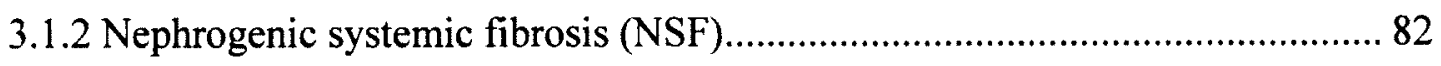

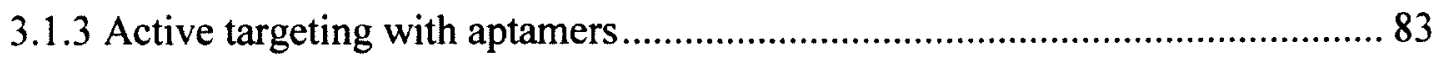

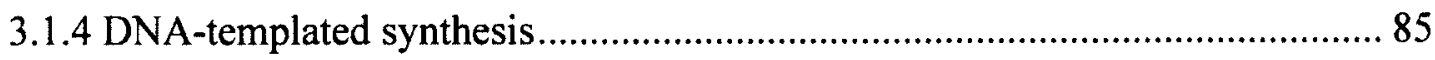

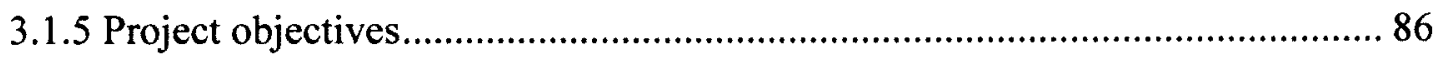

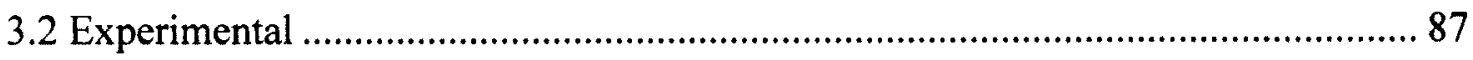

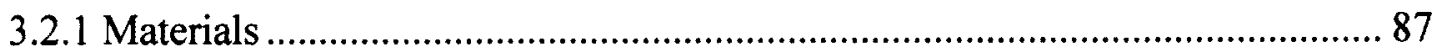

3.2.2 Synthesis of aptamer-gadolinium conjugates ................................................. 87

3.2.2.1 Oligonucleotide synthesis and modified DTPA conjugation reactions ..... 88

3.2.2.2 Purification of DNA-DTPA conjugates................................................... 90

3.2.2.3 Chelation of gadolinium(III) ……........................................................ 91

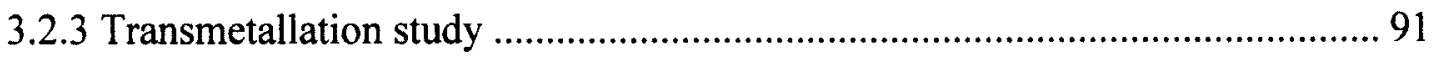

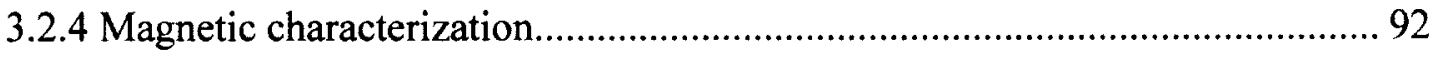

3.2.5 DNA templated synthesis .......................................................................... 92

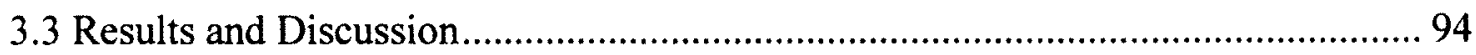

3.3.1 Synthesis of aptamer-gadolinium conjugates .................................................. 94

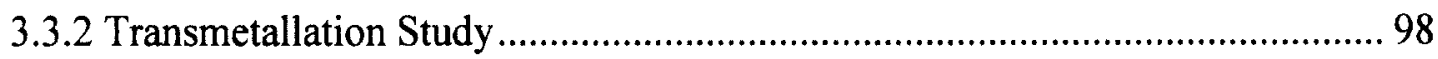

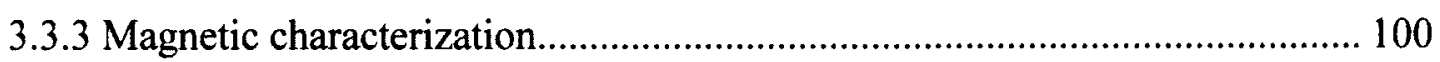

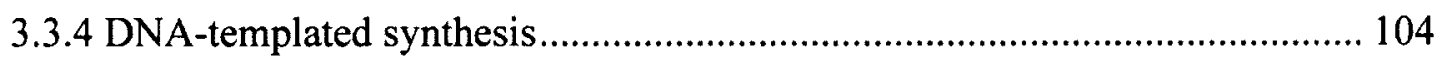

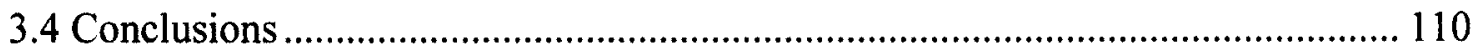

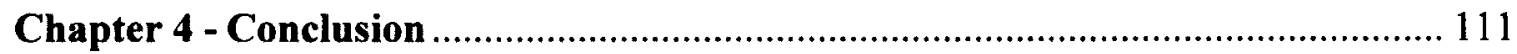

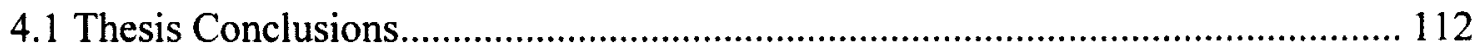

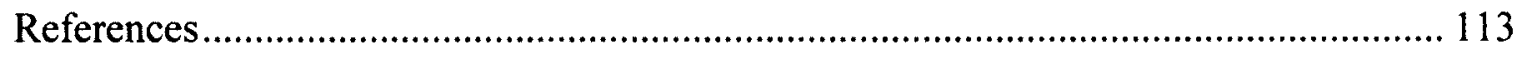




\section{List of Tables}

Table 1-1: Comparison of aptamers to related affinity probes (Adapted from Ruigrok et al. $)^{15}$

Table 2-1: Reverse phase HPLC program for isolation of MB-DNA. ............................ 35

Table 2-2: Clarity QSP protocol for purification of modified thrombin aptamer............. 38

Table 2-3: Cyclic voltammetry parameters for electrochemical cleaning of gold electrodes in sulfuric acid.

Table 2-4: Cyclic voltammetry parameters used for electrochemical characterization of DNA modified gold electrodes.

Table 2-5: Square wave voltammetry parameters used for electrochemical characterization of DNA modified gold electrodes.

Table 2-6: Chronocoulometry parameters used for electrochemical characterization of DNA modified gold electrodes.

Table 2-7: Summary of alternate methods attempted in the synthesis and purification of MB-DNA.

Table 3-1: The titration method of encouraging DNA-DTPA-DNA conjugates 90

Table 3-2: Effectiveness of adapted methods toward the production of di-substituted aptamer-DTPA conjugates.

Table 3-3: Preliminary characterization of thrombin aptamer-DTPA $\left(\mathrm{Gd}^{3+}\right)$ conjugates at $300 \mathrm{MHz}$. 101

Table 3-4: Characterization of thrombin aptamer-DTPA $\left(\mathrm{Gd}^{3+}\right)$ and thrombin aptamerpoly(T)-DTPA $\left(\mathrm{Gd}^{3+}\right)$ conjugates at $400 \mathrm{MHz}$. 


\section{List of Figures}

Figure 1-1: The Systematic Evolution of Ligands by EXponential enrichment. Repeated cycles of target incubation, removal of weakly bound candidates, and amplification of strong binding candidates enriches a random library of oligonucleotides. (adapted from Proske et. al. $)^{9}$

Figure 1-2: Selected aptamer-based sensing approaches: i) Molecular beacon-type approach with FRET pairs, ii) Colourimetric sensor employing a gold nanoparticle aggregation strategy, iii) Electrochemical sensor based on the interaction between an electrochemically active label and a gold electrode ( $\mathrm{F}=$ fluorophore, $\mathrm{Q}=$ quencher, $\mathrm{T}=$ target molecule). (adapted from Cho et al.) ${ }^{33}$

Figure 1-3: Three predominant thrombin binding aptamers. Fibrinogen exosite binders were selected by Bock et al. in 1992 and Macaya et al. in 1995. The heparin exosite binder was selected by Tasset et al. in 1997. (adapted from Lu et. al.) ${ }^{14}$ 10

Figure 1-4: Chemical DNA synthesis cycle by the phosphoramidite approach. Detritylation of a support bound nucleoside precedes coupling of a nucleoside phosphoramidite. Subsequent capping and oxidation steps yield a phosphotriester linkage. Cleavage and deprotection yields product oligonucleotides. (adapted from Gilar et. al. $)^{48}$.....

Figure 2-1: The three-electrode system. The potential profile in solution is indicated, with all relevant voltage drops between electrodes labeled. $\left(\mathrm{V}_{\mathrm{a}}=\right.$ applied voltage across working and auxiliary electrodes, $\mathrm{v}_{\mathrm{s}}=$ voltage arising from resistance of the solution, $\mathrm{v}_{\mathrm{c}}$, $\mathrm{v}_{\mathrm{r}}$, and $\mathrm{v}_{\mathrm{w}}=$ interface voltage drops at the auxiliary, reference, and working electrodes respectively). (adapted from Franklin et al.) ${ }^{64}$

Figure 2-2: i) Imposed potential waveform during the cyclic voltammetry experiment, ii) Resultant cyclic voltammogram. ( $E_{1}$ and $E_{2}=$ initial and final potential, $E_{p, c}$ and $E_{p, a}=$ cathodic and anodic peak potentials, $\mathrm{i}_{\mathrm{p}, \mathrm{c}}$ and $\mathrm{i}_{\mathrm{p}, \mathrm{a}}=$ cathodic and anodic peak currents). (adapted from Bond et al. and Forster et al. $)^{68,69}$

Figure 2-3: i) Square wave waveform showing the points at which forward and reverse currents are sampled, and the square wave period and amplitude, ii) Forward and reverse components of the square wave with the net difference indicated as a solid line. (adapted from Kounaves et al. and White et al.) $)^{70,72}$

Figure 2-4: i) Potential waveform for the chronocoulometry experiment, ii) Typical response curve. ( $E_{1}$ and $E_{2}=$ initial and final potential, $\tau=$ charge accumulation period). ${\text { (adapted from Bard et al. })^{65}}$ 
Figure 2-5: Schematic diagram for an electrochemical workstation employing the three electrode system. (adapted from Bard et al.) ${ }^{65}$....

Figure 2-6: Signal-on architectures for electrochemical DNA sensors: i) Sandwich architecture employing capture and probe-modified strands, ii) Target induced strand displacement, iii) Target induced pseudoknot disruption. (adapted from Miranda-Castro et al. $)^{20}$......

Figure 2-7: Electrocatalytic reduction of ferricyanide by methylene blue. (adapted from Farjami et al. $)^{83}$ 26

Figure 2-8: i) Interruption of charge transport for detection of a single mismatch, ii) Chronocoulometric detection of this interruption. Data corresponds to an experiment which included electrocatalytic enhancement. (adapted from Boon et al. and Kelley et al.) $)^{82,85}$

Figure 2-9: Original signal-off (i) and signal-on (ii) electrochemical aptasensor designs proposed by Xiao et al. Signal arises as binding-induced changes in conformational dynamics modify the interaction between redox-active methylene blue and the gold electrode surface. (adapted from Lubin et al.) ${ }^{32}$

Figure 2-10: An impedimetric aptasensor for lysozyme. Target-binding at the appropriate $\mathrm{pH}$ provides the charge compensation to allow an anionic redox-active probe to approach the electrode. (adapted from Willner et al.) ) $^{89}$

Figure 2-11: An electrochemical aptasensor for thrombin incorporating both the sandwich architecture and catalytic labeling concepts. (adapted from Willer et al. ${ }^{89} \ldots . .30$

Figure 2-12: Active ester conjugation of DCMB-SE to 3'- $\mathrm{NH}_{2}$ modified DNA. Hydrolysis of the second succinimidyl ester occurs during work up.

Figure 2-13: Ellman's reaction. Absorbance of the 5-thio-2-nitrobenzoic acid anion produced $\left(\mathrm{TNB}^{2-}, \varepsilon_{412 \mathrm{~nm}}=14150 \mathrm{M}^{-1} \mathrm{~cm}^{-1}\right)$ may be used for the quantification of thiol modified compounds. (adapted from Riddles et al.) ${ }^{94}$.

Figure 2-14: Cyclic voltammogram of a clean gold working electrode. 40

Figure 2-15: Moles of methylene blue removed vs. round of ethanol precipitation, quantified by absorbance at $668 \mathrm{~nm}$. Inset: UV-Visible spectra.

Figure 2-16: RP-HPLC chromatograms of unlabeled thrombin aptamer (DNA) and methylene blue conjugation products (MB-DNA). UV-signals at $260 \mathrm{~nm}$ and $668 \mathrm{~nm}$ plotted for each. 
Figure 2-17: RP-HPLC Collection at 11 minutes: i) UV-Visible spectrum, ii) Deconvoluted ESI mass spectrum

Figure 2-18: RP-HPLC Collection at 11.5 minutes: i) UV-Visible spectrum, ii) Deconvoluted ESI mass spectrum 50

Figure 2-19: Results of the Ellman reaction: i) UV-Visible spectra of the products using 1:1, 5:1, and 9:1 molar ratios of DTNB reagent to DNA, ii) Deconvoluted ESI mass spectrum of 9:1 DTNB:DNA reaction products.

Figure 2-20: Cyclic voltammograms of MB-DNA modified electrodes in $10 \mathrm{mM}$ tris with (blue) and without (red) a backfilling layer of 6-mercapto-1-hexanol (MCH). 56

Figure 2-21: Qualitative analysis of SH-DNA surface coverage by cyclic voltammetry. Response to $2 \mathrm{mM} \mathrm{K}_{3} \mathrm{Fe}(\mathrm{CN})_{6}$ of a (i) bare gold electrode, (ii) SH-DNA modified gold electrode.

Figure 2-22: Time course study by square wave voltammetry of a MB-DNA modified electrode in the presence of $350 \mathrm{nM}$ thrombin protein.

Figure 2-23: Time course study by square wave voltammetry in the presence and absence of 350nM thrombin target for both thrombin binding buffer (TBB) and its mimic (NoKTBB).

Figure 2-24: Cyclic voltammograms for (i) SH-DNA and (ii) MB-DNA modified electrodes with increasing concentrations of $\mathrm{K}_{3} \mathrm{Fe}(\mathrm{CN})_{6}$ in thrombin binding buffer (TBB).

Figure 2-25: Cyclic voltammograms of SH-DNA (red) and MB-DNA (green) modified electrodes with $0.00-0.50 \mathrm{mM} \mathrm{K}_{3} \mathrm{Fe}(\mathrm{CN})_{6}$ in TBB. The blue voltammogram corresponds to MB-DNA in the absence of $\mathrm{K}_{3} \mathrm{Fe}(\mathrm{CN})_{6}$. X-and $\mathrm{Y}$ - scales are identical in all 8 plots. 64

Figure 2-26: Cyclic voltammograms for (i) SH-DNA and (ii) MB-DNA modified electrodes with increasing concentrations of $\mathrm{K}_{3} \mathrm{Fe}(\mathrm{CN})_{6}$ in $10 \mathrm{mM}$ tris buffer.

Figure 2-27: Cyclic voltammograms of SH-DNA (red) and MB-DNA (green) modified electrodes with $0.00-0.50 \mathrm{mM} \mathrm{K}_{3} \mathrm{Fe}(\mathrm{CN})_{6}$ in $10 \mathrm{mM}$ tris buffer. The blue voltammogram corresponds to MB-DNA in the absence of $\mathrm{K}_{3} \mathrm{Fe}(\mathrm{CN})_{6}$. X- and $\mathrm{Y}$ - scales are identical in all 8 plots.

Figure 2-28: Electrocatalysis with non-covalent methylene blue $(6 \mu \mathrm{M})$ and $\mathrm{K}_{3} \mathrm{Fe}(\mathrm{CN})_{6}$ $(2 \mathrm{mM})$ in $10 \mathrm{mM}$ tris buffer. 68

Figure 2-29: Cyclic voltammograms for (i) SH-DNA and (ii) MB-DNA modified electrodes with increasing concentrations of $\mathrm{K}_{3} \mathrm{Fe}(\mathrm{CN})_{6}$ in PBS. 
Figure 2-30: Cyclic voltammograms of SH-DNA (red) and MB-DNA (green) modified electrodes with $0.00-0.50 \mathrm{mM} \mathrm{K}_{3} \mathrm{Fe}(\mathrm{CN})_{6}$ in PBS. The blue voltammogram corresponds to MB-DNA in the absence of $\mathrm{K}_{3} \mathrm{Fe}(\mathrm{CN})_{6}$. X- and $\mathrm{Y}$ - scales are identical in all 8 plots. 71

Figure 2-31: Cyclic voltammograms for increasing concentrations of thrombin in (i) PBS with $0.6 \mathrm{mM} \mathrm{KCl}$ and (ii) $\mathrm{PBS}$ with $0.2 \mathrm{mM} \mathrm{K}_{3} \mathrm{Fe}(\mathrm{CN})_{6}$. One hour incubation time preceded the acquisition for each concentration.

Figure 2-32: Integrated cathodic peaks from cyclic voltammetry experiments in the presence (red) and absence (blue) of $\mathrm{K}_{3} \mathrm{Fe}(\mathrm{CN})_{6}$

Figure 2-33: Chronocoulometry results for increasing concentrations of thrombin in PBS with $0.2 \mathrm{mM} \mathrm{K}_{3} \mathrm{Fe}(\mathrm{CN})_{6}$. Potential for the first step was set to match the cathodic peak maximum for the corresponding $\mathrm{CV}$ experiment.

Figure 2-34: Sensor responses with $0.2 \mathrm{mM} \mathrm{K}_{3} \mathrm{Fe}(\mathrm{CN})_{6}$ for cyclic voltammetry (red) chronocoulometry (green), and without $\mathrm{K}_{3} \mathrm{Fe}(\mathrm{CN})_{6}$ (blue). Replication for the purpose of generating error bars for all curves proposed as future experiments.

Figure 2-35: Regeneration of sensor surfaces via one minute soak in $8 \mathrm{M}$ guanidine hydrochloride.

Figure 3-1: Structures of common gadolinium-based MRI-CAs (i) Magnevist (gadolinium diethylenetriaminepentaacetate) and (ii) Omniscan (a bisamide derivative). (adapted from Caravan et al.) ${ }^{129}$

Figure 3-2: Structures of previously generated aptamer-gadolinium conjugates, (i) monosubstituted DTPA or 'single conjugate' (SC), (ii) di-substituted DTPA or 'double conjugate' (DC). (DNA = thrombin aptamer)

Figure 3-3: DNA-templated synthesis architectures for increasing the effective molarity of reagents A and B: (i) linear architecture, (ii) $\Omega$ architecture. (adapted from Rozenman et al. $)^{153}$

Figure 3-4: The conjugation of DTPA-dianhydride to amino-modified DNA, followed by (i) hydrolysis of the second anhydride to form a mono-substituted conjugate, or (ii) reaction with a second equivalent of amino-modified DNA at the second anhydride to form a di-substituted conjugate.

Figure 3-5: Potential reactions at the second dianhydride after initial conjugation of DTPA-DA to support bound $\mathrm{NH}_{2}$-DNA: i) Reaction with a nearby $\mathrm{NH}_{2}$-DNA yielding a di-conjugate, ii) Hydrolysis yielding a mono-conjugate, iii) Addition of a second equivalent of $\mathrm{NH}_{2}$-DNA to yield a di-conjugate. 
Figure 3-6: Poly(acrylamide) gel separation of mono- and di-substituted aptamer-DTPA conjugates. Left lanes correspond to conjugated prepared with the thrombin aptamer alone, right lanes to the $15 \mathrm{~T}$-thrombin aptamer.

Figure 3-7: UV-Vis spectra from the xylenol orange test for $\mathrm{Gd}^{3+}$. Absorbance at $\sim 570 \mathrm{~nm}$ was used to determine $\mathrm{Gd}^{3+}$ present in the aptamer-DTPA $\left(\mathrm{Gd}^{3+}\right)$ filtrate. 98

Figure 3-8: Decay in the measured relaxivity of aptamer-DTPA $\left(\mathrm{Gd}^{3+}\right)$ resulting from transmetallation by $\mathrm{Zn}^{2+}$ and precipitation of $\mathrm{Gd}^{3+}$ by $\mathrm{PO}_{4}^{-}$.

Figure 3-9: Low-field magnetic characterization of thrombin aptamer-DTPA $\left(\mathrm{Gd}^{3+}\right)$ conjugates performed with a clinical MR instrument.

Figure 3-10: Melting study for mixtures of $\mathrm{E} 10+\mathrm{T} 30$ and $\mathrm{E} 20+\mathrm{T} 30$.

Figure 3-11: Unsuccessful DNA-templated DTPA-DA conjugation reaction. Leftmost lanes contain E10, E20, and E30 markers. Lanes to the right contain oligonucleotides isolated from reaction product supernatants.

Figure 3-12: Direct PAGE characterization of DNA-templated DTPA-DA and DSS conjugation reaction mixtures.

Figure 3-13: Deconvoluted ESI mass spectra of suspected conjugates isolated by PAGE, (i) TS1: E20-DSS-T30, (ii) TS2: E10-DTPA-T30, (iii) TS3: E10-DSS-T30, (iv) TS4: E20-DSS-T30. 107

Figure 3-14: Structure of the E10-DTPA-T30 conjugate produced by DNA-templated synthesis with DTPA-DA. 108

Figure 3-15: Proposed Lossen reaction leading to the formation of interstrand $\beta$-alanine linkages. (adapted from Zalipsky 1998) ${ }^{164}$ 109 


\section{List of Abbreviations}

ATP - adenosine triphosphate

BSA - bovine serum albumin

$\mathrm{CC}$ - chronocoulometry

$\mathrm{CPG}$ - controlled pore glass

CTAB - cetyltrimethylammonium bromide

$\mathrm{CV}$ - cyclic voltammetry

DCMB-SE - Dicarboxymethylene blue NHS ester - 3,7-bis(N-(3-carboxypropyl)-N-

methylamino)-phenothiazin-5-ium perchlorate, succinimidyl ester

DNA - deoxyribonucleic acid

DMF - N,N-dimethylformamide

DMS(O)MT - 4,4'-dimethoxy-4''-methylsulfonyltrityl

DMT - dimethoxytrityl

dsDNA - double stranded DNA

DSS - disuccinimidyl suberate

DTNB - 5,5'-dithiobis-2-nitrobenzoic acid

DTPA - diethylenetriaminepentaacetic acid

DTPA-DA - diethylenetriaminepentaacetic acid dianhydride

DTPA-DNA - conjugation product of DTPA-DA and amino modified DNA

E-AB - electrochemical aptamer-based (sensor)

E-DNA - electrochemical DNA (sensor)

EDTA - ethylenediaminetetraacetic acid

ESI-MS - electrospray ionization - mass spectrometry

xiv 
fmoc - fluorenylmethyloxycarbonyl

FRET - fluorescence resonant energy transfer

HEPES - 4-(2-hydroxyethyl)-1-piperazineethanesulfonic acid

$\mathrm{K}_{\mathrm{d}}-$ dissociation constant

MB - methylene blue

MB-DNA - methylene blue conjugated to deoxyribonucleic acid

$\mathrm{MCH}$ - 6-mercapto-1-hexanol

MIP - molecularly imprinted polymer

MRI - magnetic resonance imaging

MRI-CA - magnetic resonance imaging contrast agent

NMR - nuclear magnetic resonance

NoKTBB - thrombin binding buffer mimic with no potassium or magnesium

NSF - nephrogenic systemic fibrosis

PAGE - poly(acrylamide) gel electrophoresis

PBS - phosphate buffered saline

s-PBS - phosphate buffered saline for DNA modified electrode storage

$\mathrm{PCR}$ - polymerase chain reaction

PET - polyethylene terephthalate

QCM - quartz crystal microbalance

RNA - ribonucleic acid

RP-HPLC - reversed-phase high performance liquid chromatography

SELEX - systematic evolution of ligands by exponential enrichment

SH-DNA - thiolated DNA 
SWV - square wave voltammetry

UV-Vis - ultraviolet/visible spectrophotometry

TBB - thrombin-aptamer binding buffer

TBE - tris(hydroxymethyl)aminomethane/borate/ethylenediaminetetraacetic acid buffer

TCEP - tris(2-carboxyethyl)phosphine hydrochloride

TEAA - triethylammonium acetate

TEMED - N,N,N',N'-tetramethylethylenediamine

TNB - 5-thio-2-nitrobenzoic acid

tris - tris(hydroxymethyl)aminomethane

UV-Vis - Ultraviolet-visible spectrophotometry

VEGF - vascular endothelial growth factor

Xylenol orange - 3,3'-bis-[N,N-di(carboxymethyl)aminomethyl]-o-cresol

sulphonephthalein 


\section{Chapter 1 - Introduction}




\subsection{Aptamers}

Aptamers are short single stranded synthetic oligonucleotides (DNA or RNA) that fold into unique molecular structures to bind specific targets with high affinity ${ }^{1}$. Aptamers may distinguish minor structural differences among target analogues, as well as discriminate between enantiomeric configurations of targets ${ }^{2,3}$. The process by which aptamers are generated was reported independently by three laboratories in $1990^{4-6}$. The process was termed "systematic evolution of ligands by exponential enrichment" (SELEX) by Tuerk and Gold ${ }^{4}$, while the products were named "aptamers" by Ellington and Szostak ${ }^{5}$.

The SELEX process begins with a library of random nucleotide sequences, 30$70 \mathrm{nt}$ in length, flanked by fixed regions required for amplification and cloning. The library is then subjected to repeated cycles of incubation with the target, a partitioning and removal of weakly bound sequences, and amplification of strong binding candidates by polymerase chain reaction (PCR). For larger targets such as proteins and viruses the partitioning may be done by filtration, while selections for small molecule targets often utilize target-modified affinity columns ${ }^{7}$. Due to practical limitations of DNA synthesis, SELEX libraries generally contain $10^{13}$ to $10^{15}$ sequences. While representing a tiny fraction of those theoretically possible for modestly sized random regions, this remains a sufficiently large population to contain selective, high affinity binders ${ }^{8}$. 


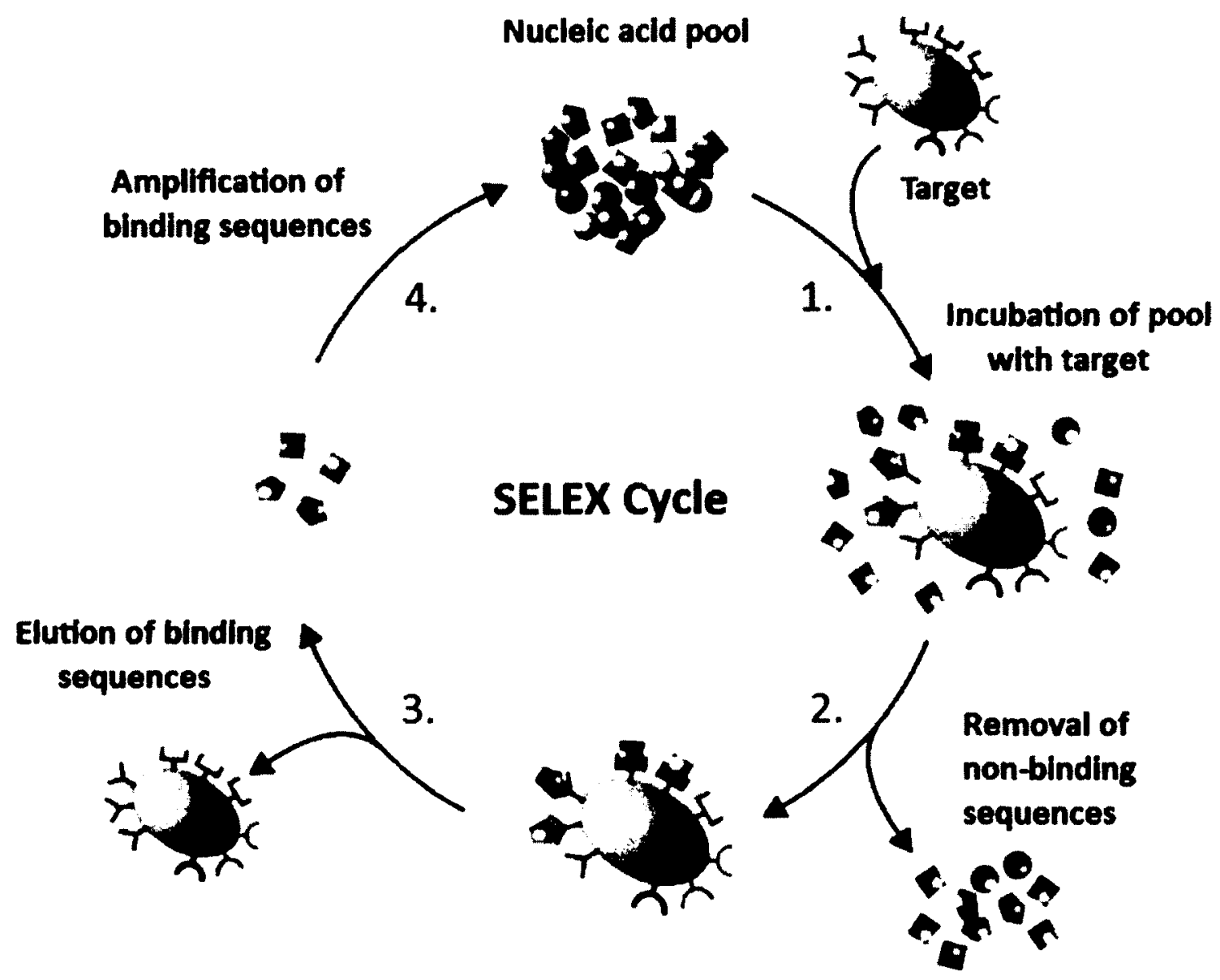

Figure 1-1: The Systematic Evolution of Ligands by EXponential enrichment. Repeated cycles of target incubation, removal of weakly bound candidates, and amplification of strong binding candidates enriches a random library of oligonucleotides. (adapted from Proske et. al.) ${ }^{9}$

When the library is sufficiently enriched in strong binding candidates, generally after 5-15 cycles, the products are cloned, sequenced, and analyzed for binding characteristics $^{8}$. Of particular importance are the dissociation constant and rates of formation and dissociation of aptamer-target complexes. The dissociation constant $\left(\mathrm{K}_{\mathrm{d}}\right)$ represents the target concentration at which half of the present aptamers will be bound, a reflection of the affinity of the aptamer for the target. The association and dissociation 
rate constants, $k_{\text {on }}$ and $k_{\text {off }}$ respectively, are indicative of the time required for aptamertarget complexes to form, and how long they persist ${ }^{10,11}$.

As alluded to above, the selectivity of aptamers among target and analogues is remarkable due to the selection pressure imposed by SELEX to form unique three dimensional binding structures ${ }^{12}$. For instance, an aptamer selected for theophylline exhibits a four order of magnitude reduction in affinity for caffeine, which differs by only a methyl group ${ }^{12}$. Similarly, an aptamer selected for L-arginine demonstrates a similar loss in affinity (12000-fold) for D-arginine ${ }^{13}$.

\subsection{Comparison of aptamers to related affinity probes}

As aptamers may be considered the nucleic acid equivalent of antibodies, comparisons are often drawn between the two ${ }^{14}$. While it must be conceded that antibodies have been far more widely applied, there are a number of advantages to aptamers which make them an attractive alternative ${ }^{15}$.

First, in vitro SELEX can be applied to a seemingly limitless range of targets, and accordingly aptamers have been selected for small molecules, proteins, viruses, and cells $^{16}$. Antibodies on the other hand require a particular immunogenic response from an animal. If this response is either too weak or too strong, the process of raising antibodies becomes increasingly difficult ${ }^{17}$. Antibodies are also more difficult to produce at a large scale, and animal products are subject to bacterial and viral contamination ${ }^{18}$. Additionally, the batch to batch variation in antibody activity is a not an issue for aptamer production where exact replicates of a particular sequence are generated ${ }^{19}$. 
Unlike antibodies, aptamers are also fully capable of reversible denaturation by heat, $\mathrm{pH}$, or salt concentration with no change in binding characteristics ${ }^{19}$. This is a particularly important feature when discussing reusability in analytical applications or for use as molecular switches. Aptamers are also much more resistant to non-physiological conditions and considerably more stable over time ${ }^{20}$. Furthermore, chemical modifications such as amino, thiol, and carboxyl groups may be incorporated into aptamer sequences with comparative ease and no resultant change in target recognition capability $^{21,22}$. Binding heterogeneity and an overall reduction in activity is often introduced when performing such modifications on antibodies ${ }^{15,18}$.

Due to their rising prominence in affinity-based sensors, it is also worthwhile to draw some comparisons between molecularly imprinted polymers (MIPs) and aptamers. As with aptamers, MIPs are now commonly regarded as excellent antibody alternatives for a similarly wide array of applications ${ }^{23}$. In an interesting example, Ulyanova et al. prepared a MIP for theophylline, and employed it for selective electrochemical detection in the presence of caffeine ${ }^{24}$. The resemblance of this work to the flagship example of aptamer selectivity is clear, and reflects the growing degree of competition among these related affinity probes ${ }^{15}$.

Briefly, MIPs are produced when a polymerization reaction is carried out in the presence of a target for which the monomers have a degree of affinity ${ }^{25}$. Following removal of the target, cavities remain which are complementary to the target both spatially, and in terms of exposed functional groups ${ }^{26}$. When reintroduced, the affinity and selectivity of these cavities for target molecules are comparable to those of aptamers and antibodies ${ }^{27}$. As synthetic polymers, MIPs carry their own set of advantages and 
disadvantages when an affinity probe is being selected for a particular application. Antibodies, aptamers, and MIPs are compared across several dimensions in Table 1-1.

Table 1-1: Comparison of aptamers to related affinity probes (Adapted from Ruigrok et al. $)^{15}$

\begin{tabular}{|c|c|c|c|}
\hline Metric & Aptamers & Antibodies & MIPs \\
\hline Selection & In vitro & In vivo & In vitro \\
\hline Production & Synthetic & $\begin{array}{l}\text { Animals, } \\
\text { recombinant }\end{array}$ & Synthetic \\
\hline Modification & $\begin{array}{l}\text { Straightforward, } \\
\text { terminus or internal } \\
\text { modifications } \\
\text { possible }\end{array}$ & $\begin{array}{l}\text { Possible, produces } \\
\text { heterogeneous } \\
\text { products }\end{array}$ & $\begin{array}{l}\text { Possible, can } \\
\text { complicate } \\
\text { imprinting } \\
\text { procedure }\end{array}$ \\
\hline Shelf life & $\begin{array}{l}\text { DNA: years; RNA: } \\
\text { months at }-80^{\circ} \mathrm{C} \text {, in } \\
\text { the absence of } \\
\text { nucleases }\end{array}$ & Weeks at $-4^{\circ} \mathrm{C}$ & Years. \\
\hline Target class & $\begin{array}{l}\text { Small molecules, } \\
\text { macromolecules, } \\
\text { cells }\end{array}$ & $\begin{array}{l}\text { Must be } \\
\text { immunogenic. } \\
\text { Cannot be } \\
\text { exceedingly toxic }\end{array}$ & $\begin{array}{l}\text { Primarily small } \\
\text { molecules }\end{array}$ \\
\hline $\begin{array}{l}\text { Application } \\
\text { conditions }\end{array}$ & $\begin{array}{l}\text { Physiological, non- } \\
\text { physiological } \\
\text { (selection } \\
\text { conditions) }\end{array}$ & $\begin{array}{l}\text { Physiological } \\
\text { conditions }\end{array}$ & $\begin{array}{l}\text { Primarily organic } \\
\text { solvents }\end{array}$ \\
\hline In vivo half life & $\begin{array}{l}\text { Without } \\
\text { modification: } \\
\text { seconds to minutes. } \\
\text { Modified: days }\end{array}$ & Days to weeks & $\begin{array}{l}\text { Very few studies }{ }^{28} \text {. } \\
\text { Cleared from blood } \\
\text { and sequestered in } \\
\text { liver within } 10 \\
\text { minutes }^{23}\end{array}$ \\
\hline
\end{tabular}




\subsection{Applications of aptamers}

Due to their high affinity, selectivity, and comparative advantages over related recognition elements in many situations, aptamers possess great potential in diagnostic, analytical, and therapeutic applications ${ }^{18,29,30}$.

Strong binding characteristics and ease of modification make aptamers ideal candidates for sensing in the fields of food inspection, point-of-care diagnostics, and toxin detection ${ }^{31}$. Aptamers which undergo significant conformational changes to bind with their targets, and are modified in a way that makes the measurement of these changes straightforward, are particularly useful as signal transducers ${ }^{32}$. Sensors based on aggregation or disaggregation of aptamer-labeled particles as a consequence of aptamer binding are prevalent in the literature ${ }^{33}$. In the case of gold nanoparticles, the surface plasmon resonance related optical properties are significantly changed upon aggregation, enough to construct colourimetric sensors ${ }^{33,34}$. In a molecular beacon-type approach, aptamers can be distally tagged with fluorescence resonant energy transfer (FRET) pairs whose proximity and thus signaling are controlled by target binding ${ }^{33}$. Similar principles may be applied to aptamers modified with electroactive probes, where interaction between probe and an electrode is modulated by target binding ${ }^{35}$. These electrochemical sensors will be further discussed in Chapter 2 . 
i)

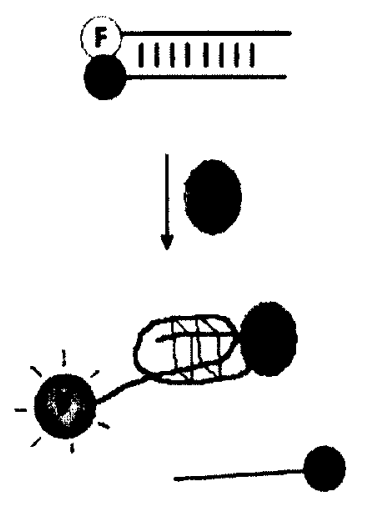

ii)
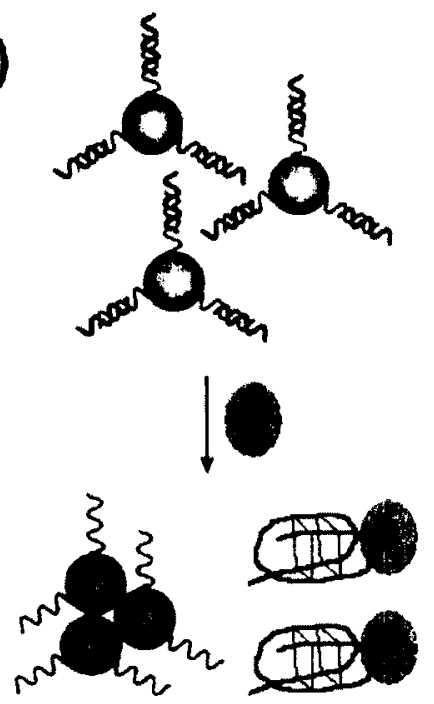

iii)

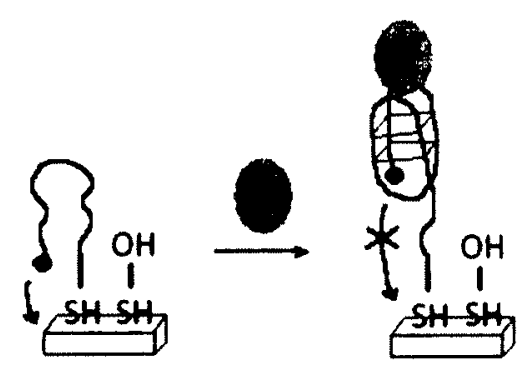

Figure 1-2: Selected aptamer-based sensing approaches: i) Molecular beacon-type approach with FRET pairs, ii) Colourimetric sensor employing a gold nanoparticle aggregation strategy, iii) Electrochemical sensor based on the interaction between an electrochemically active label and a gold electrode $(\mathrm{F}=$ fluorophore, $\mathrm{Q}=$ quencher, $\mathrm{T}=$ target molecule). (adapted from Cho et al.) ${ }^{33}$

Aptamers that are selected to protein targets often inhibit the function of that protein in vivo, thus offering utility for therapeutic applications ${ }^{18}$. Perhaps the most prolific example is pegaptanib, a modified RNA aptamer that acts as a vascular endothelial growth factor (VEGF) antagonist ${ }^{36}$. Sold under the trade name Macugen, this aptamer represents the first intravitreal therapy approved by the Food and Drug Administration for treatment of age related macular degeneration ${ }^{36}$. Several other aptamers are in clinical trials for a wide range of indications, and research into improved vectors for aptamer delivery to intracellular environments continues to progress ${ }^{16}$.

In addition to blocking protein function, other major therapeutic uses of aptamers under investigation include molecular targeting and drug delivery ${ }^{37}$. Hicke et al. described the in vivo targeting of a number of human tumors including breast cancer and 
glioblastoma using modified tenascin-C aptamers. Significant accumulation of the aptamers in mouse xenografted tumors was observed, and both fluorescent and radioactive aptamer tags were successfully employed for tumor imaging ${ }^{37}$. The enormous potential attached to this type of work provided the motivation for the project to be described in Chapter 3.

\subsection{Thrombin binding aptamers}

An exceedingly popular target for proof-of-principle experiments of novel aptamer-based sensing architectures is the enzyme thrombin ${ }^{14}$. Three prominent thrombin DNA aptamers may be found in the literature. The first thrombin aptamer was selected by Bock et al. in 1992, a 15-mer that binds to the fibrinogen-recognition site of thrombin ${ }^{38,39}$. It was quickly deduced that this aptamer forms a unimolecular Gquadruplex structure to bind with thrombin, with a $\mathrm{K}_{d}$ of approximately $100 \mathrm{nM}^{14,40}$. Two years later, Macaya et al. noted that the highest affinity thrombin aptamers were not necessarily the best inhibitors of thrombin activity in vivo ${ }^{41}$. Consequently, they identified that for an aptamer with a duplex bridging the $3^{\prime}$ and 5 ' ends of the quadruplex structure both inhibition and stability to nucleases were improved ${ }^{41}$. Furthermore, the affinity was improved by an order of magnitude by this duplex bridging $\left(K_{d}=10\right.$ $25 \mathrm{nM})^{14}$.

Tasset et al. repeated the selection of thrombin aptamers using nitrocellulose filtration as the partitioning method, rather than the agarose-concanavalin A columns used by Bock and Macaya ${ }^{42}$. From this selection, even higher affinity binders $\left(K_{d}=\right.$ 
$0.5 \mathrm{nM}$ ) arose, which were found to bind to the heparin recognition exosite of thrombin ${ }^{14}$.

The structures of the three principle thrombin aptamers are shown in Figure 1-3.

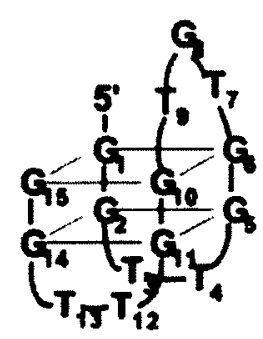

Bock

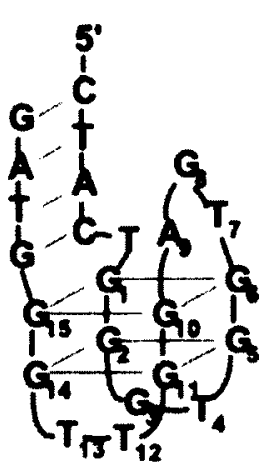

Macaya

Fibrinogen recognition exosite

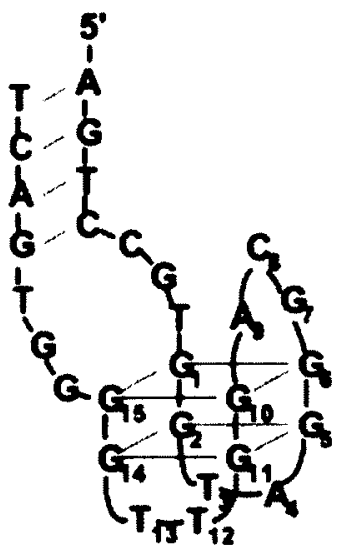

Tasset

Heparin binding exosite

Figure 1-3: Three predominant thrombin binding aptamers. Fibrinogen exosite binders were selected by Bock et al. in 1992 and Macaya et al. in 1995. The heparin exosite binder was selected by Tasset et al. in 1997. (adapted from Lu et. al.) ${ }^{14}$ 


\subsection{Chemistry of DNA Synthesis}

An additional factor which makes aptamers particularly attractive has been the concurrent development of methods for inexpensive, high throughput generation of customized oligonucleotides ${ }^{20}$. Nucleic acids are susceptible to a wide range of chemical reactions, thus complicating their synthesis beyond the simple formation of $3^{\prime}-5$ ' phosphodiester bonds between nucleotide units, and necessitating the inclusion of several protecting groups removed only at specific stages ${ }^{43}$.

A mere two years after Watson and Crick's deduction of the double helix structure of DNA, the first chemical synthesis of a dinucleotide containing a phosphate linkage identical to natural DNA was published ${ }^{44,45}$. Synthesis approaches have continued to improve to the present day, with significant land marks including the first synthesis of a complete, functional gene in the 1970 s, and a transition to solid support based methods in the $1980 \mathrm{~s}^{45}$.

Another key development in the 1980s was the introduction of the phosphoramidite approach, which made DNA synthesis more widely accessible by both allowing the building blocks to be stored for extended periods and reducing the stringency of the conditions under which DNA could be synthesized ${ }^{46}$. Furthermore, the ease of preparing modified phosphoramidite derivatives allowed straightforward introduction of chemical modifications into DNA ${ }^{47}$. Phosphoramidite chemistry, having now been fully automated, remains the current standard for DNA synthesis. 


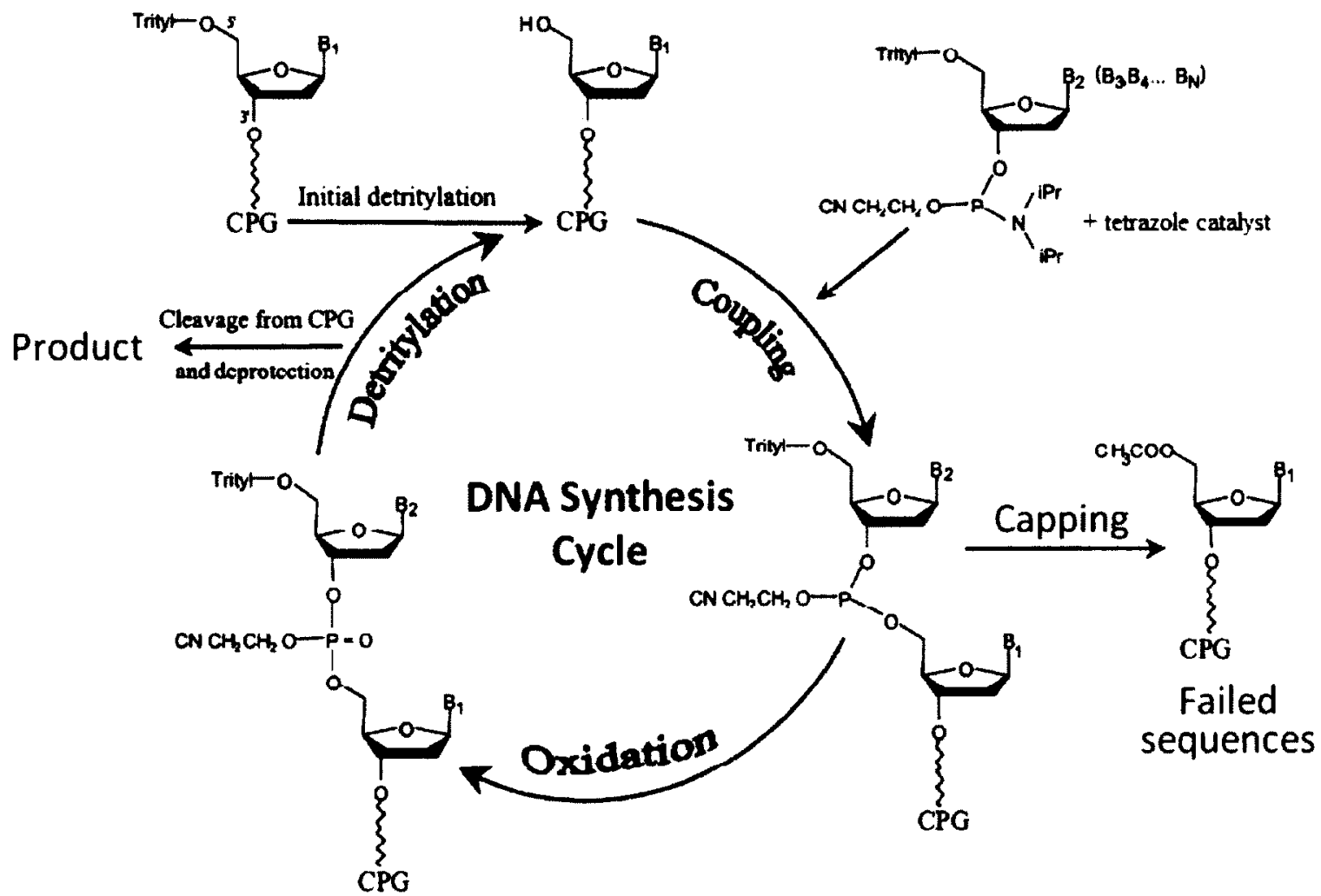

Figure 1-4: Chemical DNA synthesis cycle by the phosphoramidite approach. Detritylation of a support bound nucleoside precedes coupling of a nucleoside phosphoramidite. Subsequent capping and oxidation steps yield a phosphotriester linkage. Cleavage and deprotection yields product oligonucleotides. (adapted from Gilar et. al. $)^{48}$

As described in Figure 1-4, the DNA synthesis cycle begins with removal of the dimethoxytrityl (DMT) protecting group from the $5^{\prime}-\mathrm{OH}$ of a nucleoside bound to a solid support. The next deoxynucleoside phosphoramidite and a tetrazole catalyst are added simultaneously to form a dinucleoside phosphite. Unreacted 5'-OH groups are capped with acetic anhydride to minimize the length of failed sequences. The capping reaction has the added benefit of reversing undesired phosphitylation of guanine during the coupling step ${ }^{49}$. Finally, the phosphite linkage is oxidized to a phosphate triester. The 
cycle is repeated, adding each deoxynucleoside phosphoramidite in the appropriate order to produce the desired sequence. Sequences are cleaved from the solid support, and all protecting groups are removed simultaneously by treatment in aqueous ammonia ${ }^{43}$.

Reversed phase high performance liquid chromatography (RP-HPLC) allows straightforward purification of the oligonucleotides if 5'-DMT groups are retained on the final product ${ }^{48}$. Denaturing poly(acrylamide) gel electrophoresis (PAGE) may also be used to separate the oligonucleotide products by charge and ability to migrate through the polymer, particularly when the pore size approaches the size of the oligonucleotides of interest $^{50}$. Quantification by ultraviolet-visible spectrophotometry (UV-Vis) and characterization by techniques such as electrospray ionization mass spectrometry (ESIMS) are common ${ }^{43}$.

\subsection{Thesis Objectives}

This thesis will contribute to both analytical and therapeutic applications of aptamer technology. The second chapter of this thesis will present work done towards the electrocatalytic enhancement of an established electrochemical aptamer based sensor for thrombin. To this end, the development of a protocol for synthesizing the required probe labeled aptamer in house will be described. Optimization of the thrombin sensor, and its performance under electrocatalytic conditions will be discussed. The third chapter will detail the improved synthesis and characterization of new aptamer-gadolinium conjugates proposed as MRI contrast agents. First steps to a simple transition into the field of "theragnostics" for these conjugates will also be covered. 
Chapter 2 - The enhancement of electrochemical aptasensors via probe electrocatalysis 


\subsection{Introduction}

\subsubsection{Biosensors}

Biosensors are devices which determine the presence of a target molecule through interaction with a biological recognition mechanism, and the measurement of resultant changes in gravimetric, electronic, thermal, or optical signals ${ }^{51,52}$. Biosensors may be categorized by the nature of the recognition element, as those which feature catalytic activity versus simple high-affinity binders ${ }^{53}$. The former take advantage of the high selectivity of enzymes to catalyze particular chemical reactions, either generating or consuming a detectable species ${ }^{54}$. By far, the most successful of all biosensors is the home glucose monitor used by millions of diabetics worldwide, based on the activity of glucose oxidase ${ }^{32,55}$. As other examples, alcohol dehydrogenase, cholesterol oxidase, and polyphenol oxidase have been used to prepare catalytic biosensors for ethanol, cholesterol, and dopamine respectively ${ }^{54}$.

Affinity biosensors on the other hand utilize selective binding of the recognition element to its target ${ }^{54}$. DNA-based sensors which detect their complementary sequence are popular due to applicability in the areas of gene analysis and forensics ${ }^{56}$. Aptamers are particularly intriguing recognition elements for a wider range of targets due to their high affinity and selectivity ${ }^{52}$. The comparative ease with which aptamers may be modified with optically or electrically active probes, as well as various functional groups for immobilization on a variety of surfaces contributes to their attractiveness in biosensing ${ }^{32}$. Aptamer selections can be performed under conditions that mimic the

eventual sample matrix, which is particularly useful for environmental or food analysis ${ }^{57}$. 
Furthermore, $k_{\text {on }}$ and $k_{\text {off }}$ rates may be tailored during selection to suit the needs of the desired assay ${ }^{57}$.

The first biosensors utilizing aptamers ('aptasensors') were introduced in the late 1990 s, and were based on optical detection schemes ${ }^{58}$. In a more recent example, Li and coworkers immobilized fluorescently tagged thrombin aptamer onto surfactant dispersed graphene. Graphene was observed to quench the fluorescent tag, however fluorescence was restored as aptamers preferentially bound to added thrombin target, dissociating from graphene $^{59}$. Fluorescence polarization was used by Zhu et al. in a signal-off sensor design for the small molecule L-tyrosinamide. L-tyrosinamide aptamers were hybridized with short fluorescently tagged segments of their complementary sequence. Introduction of the target disrupted aptamer-complement duplexes via preferential formation of aptamertarget complexes. The released complementary segments showed a significantly decreased fluorescence anisotropy signal as a consequence of the lessened mass in comparison to the hybrids ${ }^{60}$.

Aptasensors have also been constructed using mass based detection, specifically with the quartz crystal microbalance $(\mathrm{QCM})^{58}$. QCM is based on a piezoelectric crystal sandwiched between two electrodes which impose an electric field ${ }^{54}$. The resonant frequency of the crystal is exquisitely sensitive to mass changes at the surface, such as those that result from target molecules binding to electrode bound aptamers ${ }^{61}$. Zhang et al. demonstrated the utility of aptamer modified quartz crystals for the detection of both immunoglobulin $\mathrm{E}$ and $\mathrm{VEGF}^{61}$. Similarly, Mascini and coworkers prepared a QCM based aptasensor for HIV-1 Tat protein ${ }^{62}$. 
Although optical and mass based detection mechanisms have been successful academically, interest in electrochemical aptasensors has been expanding rapidly due to factors such as low cost, simplicity, and portability ${ }^{58}$. Accordingly, electrochemical biosensors will be discussed in more detail in the subsequent sections.

\subsubsection{Electrochemistry}

As is evident from the etymology, electrochemistry is the study of the interaction between electricity and chemistry. The analytical chemist is particularly interested in the measurement of electrical quantities such as current, charge, and potential, and relating them to chemical parameters of a solution ${ }^{54}$. Electroanalytical techniques are frequently categorized based on the particular electrical quantity being measured. For instance, potentiometric sensors measure potential of systems at equilibrium, while amperometric sensors measure current as reactions are driven at a known potential ${ }^{63}$.

Electrochemical processes take place at the heterogeneous interface of electrode and solution ${ }^{54}$. At equilibrium with no current flow, charge builds up on both the solid and liquid sides of the interface, forming what is referred to as the electrical double layer $^{64}$. Resembling a parallel plate capacitor, the electrical double layer is charged

during measurements, resulting in a background current known as the charging current ${ }^{54}$. The charging current can overwhelm the faradaic current from electrochemical reactions of interest when concentrations are very small, although this can be minimized by using microelectrodes ${ }^{65,66}$. 


\subsubsection{Three electrode system}

Two electrodes are required to complete an electrochemical cell, a working electrode at which electrochemical reactions of interest take place, and an inert auxiliary electrode to complete the electrochemical circuit ${ }^{65}$. However, the three electrode system is commonly used in electroanalysis, and has key advantages over systems containing only a working and auxiliary electrode ${ }^{64}$. Three electrode systems add a separate electrode to serve as a reference from which the potential of the working electrode is measured $^{65}$. The potential drops between electrodes in the three electrode system are shown in Figure 2-1.

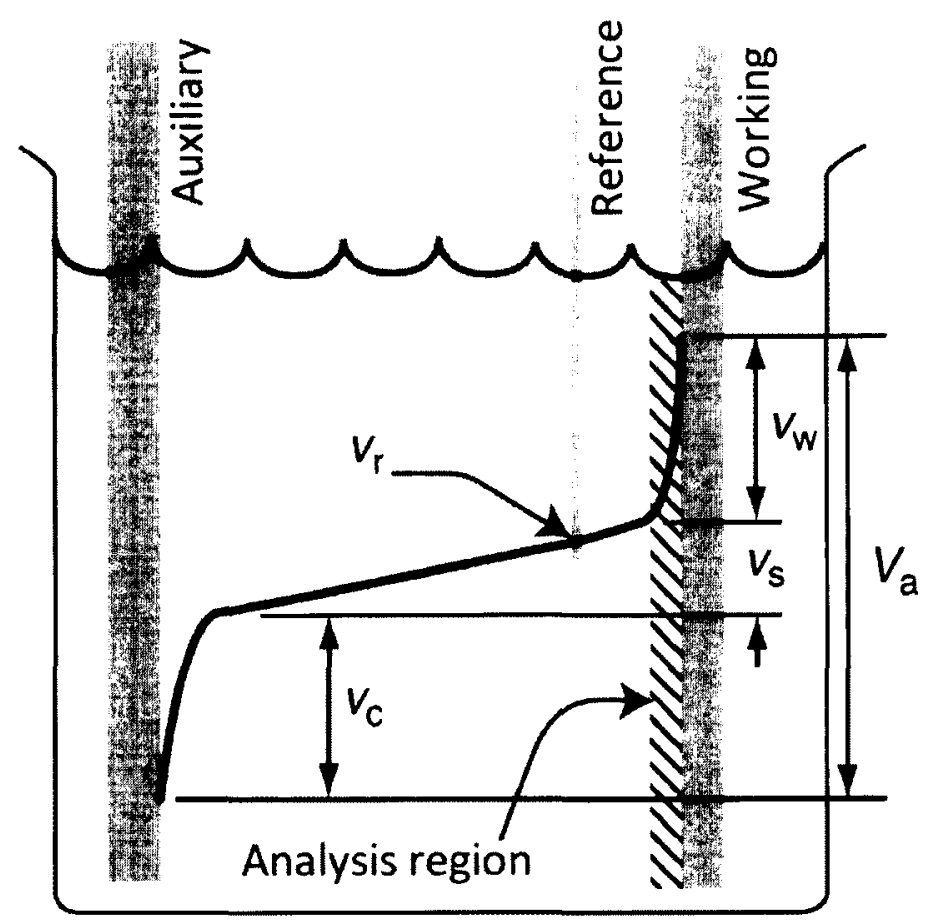

Figure 2-1: The three-electrode system. The potential profile in solution is indicated, with all relevant voltage drops between electrodes labeled. $\left(V_{\mathrm{a}}=\right.$ applied voltage across working and auxiliary electrodes, $v_{s}=$ voltage arising from resistance of the solution, $v_{c}, v_{r}$, and $v_{w}=$ interface voltage drops at the auxiliary, reference, and working electrodes respectively). (adapted from Franklin et al.) ${ }^{64}$ 
For analytical applications, processes occurring at the surface of the working electrode are of interest, and thus accurate measurement of the voltage at the interface of the working electrode, $v_{w}$, is required. When using a two electrode system however, only the entire applied potential between working and auxiliary electrodes, $V_{a}$ can be measured. By adding the third reference electrode, $\mathrm{v}_{\mathrm{c}}$, the potential drop at the auxiliary electrode is eliminated. The potential drop due to resistance of the solution, $\mathrm{v}_{\mathrm{s}}$, remains and can introduce some error into the assumption that $\mathrm{v}_{\mathrm{w}} \approx \mathrm{v}_{\mathrm{r}}$. The influence of $\mathrm{v}_{\mathrm{s}}$ is minimal at higher ionic strengths due to decreased resistance of the solution ${ }^{64}$. The factor by which $v_{r}$ and $v_{w}$ differ can be further minimized by placing the reference electrode as close as possible to the working electrode, as is often done with a Luggin capillary ${ }^{65}$. Additionally, modern electrochemical instruments feature additional circuitry which compensates for the difference between $v_{r}$ and $v_{w}{ }^{65}$.

\subsubsection{Electrochemical techniques}

Cyclic voltammetry (CV) is a widely used technique for studying electrochemical processes involving both organic and inorganic compounds ${ }^{67}$. The technique is very simple to perform, but provides a large amount of information about electrochemical reactions $^{67}$. In a $\mathrm{CV}$ experiment, a potential is applied to an electrochemical cell at a constant rate, once a predetermined switching potential is reached, the potential is swept back to the initial value. The potential waveform and the corresponding expected result are shown in Figure 2-2. 

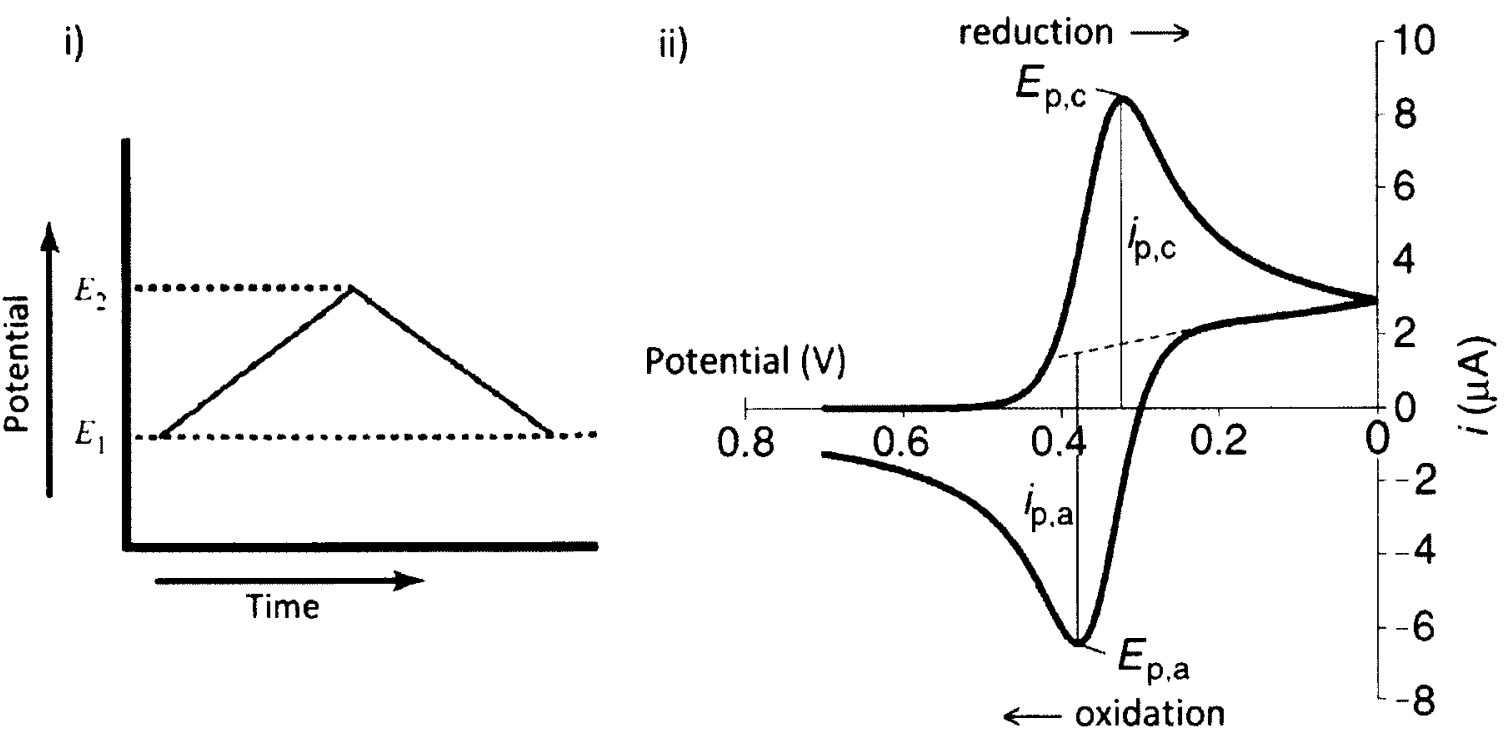

Figure 2-2: i) Imposed potential waveform during the cyclic voltammetry experiment, ii) Resultant cyclic voltammogram. $\left(E_{1}\right.$ and $E_{2}=$ initial and final potential, $E_{p, c}$ and $E_{p, a}=$ cathodic and anodic peak potentials, $i_{p, c}$ and $i_{p, a}=$ cathodic and anodic peak currents). (adapted from Bond et al. and Forster et al. $)^{68,69}$

For the experiment shown in Figure 2-2, the initial potential $E_{1}$ is set to $0.7 \mathrm{~V}$, and $\mathrm{E}_{2}$ set to $0 \mathrm{~V}$, and the reaction $\mathrm{O}+\mathrm{ne}^{-} \rightleftharpoons \mathrm{R}$ is a reversible process. Scanning in the negative direction, current increases as sufficient potential is supplied to drive the reaction in the forward direction, forming a cathodic peak at $E_{p, c}$. The potential is swept back in the opposite direction, driving the oxidation and producing the anodic peak, $\mathrm{E}_{\mathrm{p}, \mathrm{a}}$. Other parameters of interest are $i_{p, c}$ and $i_{p, a}$, the cathodic and anodic peak currents respectively ${ }^{69}$.

Square wave voltammetry (SWV) is another versatile method, which offers a short analysis time and excellent sensitivity ${ }^{70}$. Also, SWV discriminates very well against the charging current background ${ }^{71}$. In the SWV experiment, an underlying staircase scan 
has a square wave superimposed, with sampling of both forward and reverse current samples as indicated in Figure $2-3^{65}$.
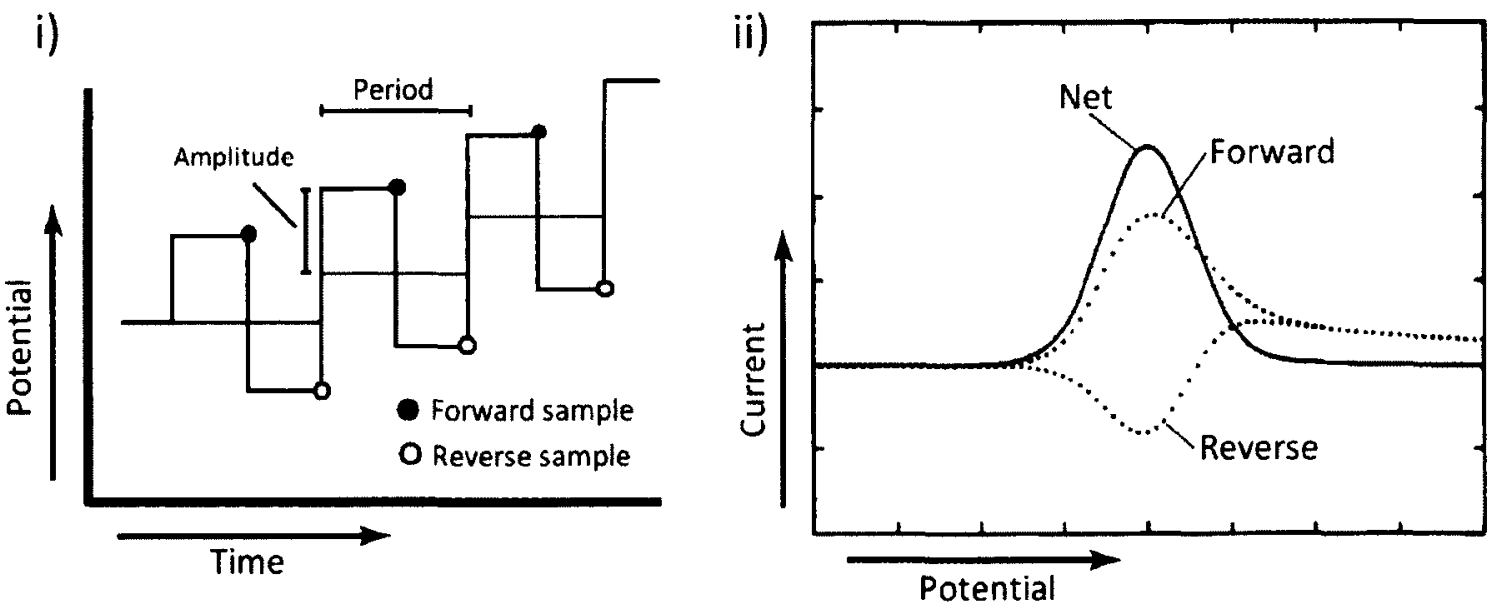

Figure 2-3: i) Square wave waveform showing the points at which forward and reverse currents are sampled, and the square wave period and amplitude, ii) Forward and reverse components of the square wave with the net difference indicated as a solid line. (adapted from Kounaves et al. and White et al.) ${ }^{70,72}$

Chronoamperometry is the monitoring of current as a function of time at a constant imposed potential. Chronocoulometry (CC), may be considered the integral analogue technique of chromoamperometry as it measures charge accumulation over time at constant potentials. In double potential step $\mathrm{CC}$ experiments, the potential is returned to its original state. Considering the waveform shown in Figure 2-4, the charge that is built while the electrode is held at $E_{2}$ is withdrawn when the potential is reverted to $E_{1}{ }^{65}$. A key drawback of the $\mathrm{CC}$ experiment is poor selectivity, as the accumulated charge represents the sum of all processes happening at the electrode, not necessarily only the reaction of interest ${ }^{64}$. 

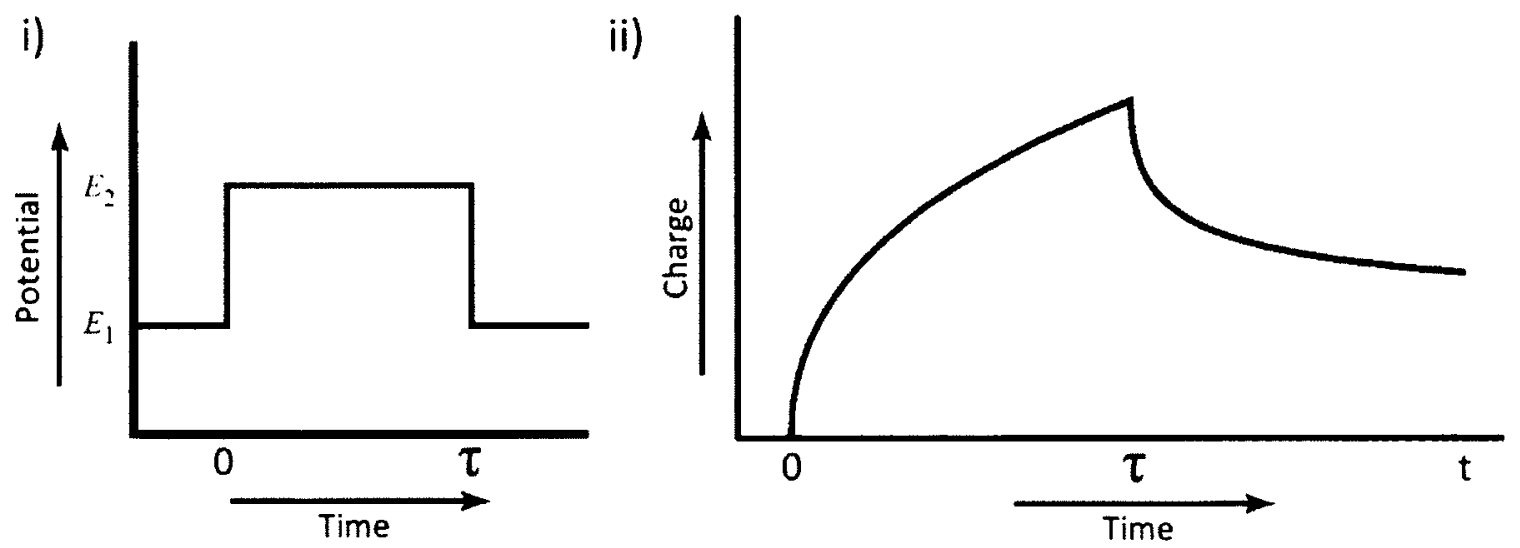

Figure 2-4: i) Potential waveform for the chronocoulometry experiment, ii) Typical response curve. $\left(E_{1}\right.$ and $E_{2}=$ initial and final potential, $\tau=$ charge accumulation period). (adapted from Bard et al.) ${ }^{65}$

$\mathrm{CV}, \mathrm{SWV}, \mathrm{CC}$, and many other techniques are accessible with an electrochemical workstation based on the three electrode system. In the typical electrochemical workstation, a potentiostat controls the voltage across the auxiliary and working electrode pair to provide the desired potential difference between the reference and working electrode. The target potential differences between the reference and working electrode are dictated by the waveform generator in accordance with the particular experiment ${ }^{65}$. Miniaturized potentiostats, capable of performing the above experiments and costing less than one hundred dollars to produce, can be deployed in resource poor environments such as remote areas, developing countries, and undergraduate teaching laboratories ${ }^{73}$. 


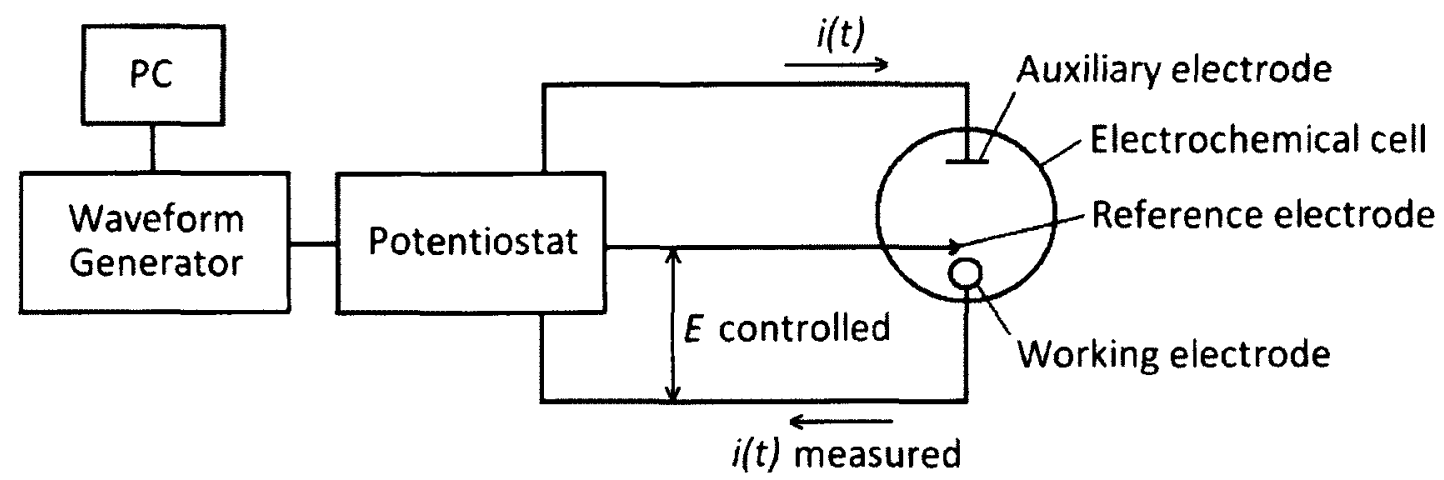

Figure 2-5: Schematic diagram for an electrochemical workstation employing the three electrode system. (adapted from Bard et al.) ${ }^{65}$

\subsubsection{Electrochemical DNA sensors}

Electrochemical DNA (E-DNA) sensors use the highly selective nature of DNA hybridization to detect analyte DNA without the burden and expense of light sources, optics, and photodetectors required by traditional optical molecular beacons ${ }^{74}$. Although studies on the direct electrochemistry of DNA had been conducted since the 1960s, the first electrochemical sensor for DNA was introduced by Millan et al. in 1993 ${ }^{34,75,76}$. In this sensor, electroactive intercalator probes were used to distinguish a single stranded poly $(\mathrm{T})$ probe immobilized on a carbon electrode from the hybrid produced upon introduction of a poly(A) target ${ }^{76}$. Secondary structures such as quadruplexes, hairpins, and pseudoknots have largely replaced linear DNA probes, for reasons such as enhanced selectivity ${ }^{20}$. Figure 2-6 outlines selected structure switching architectures for E-DNA sensors. These sensors can be constructed by immobilizing redox-labelled oligonucleotides onto gold electrodes through the widely used gold-thiol interaction ${ }^{77,78}$. 

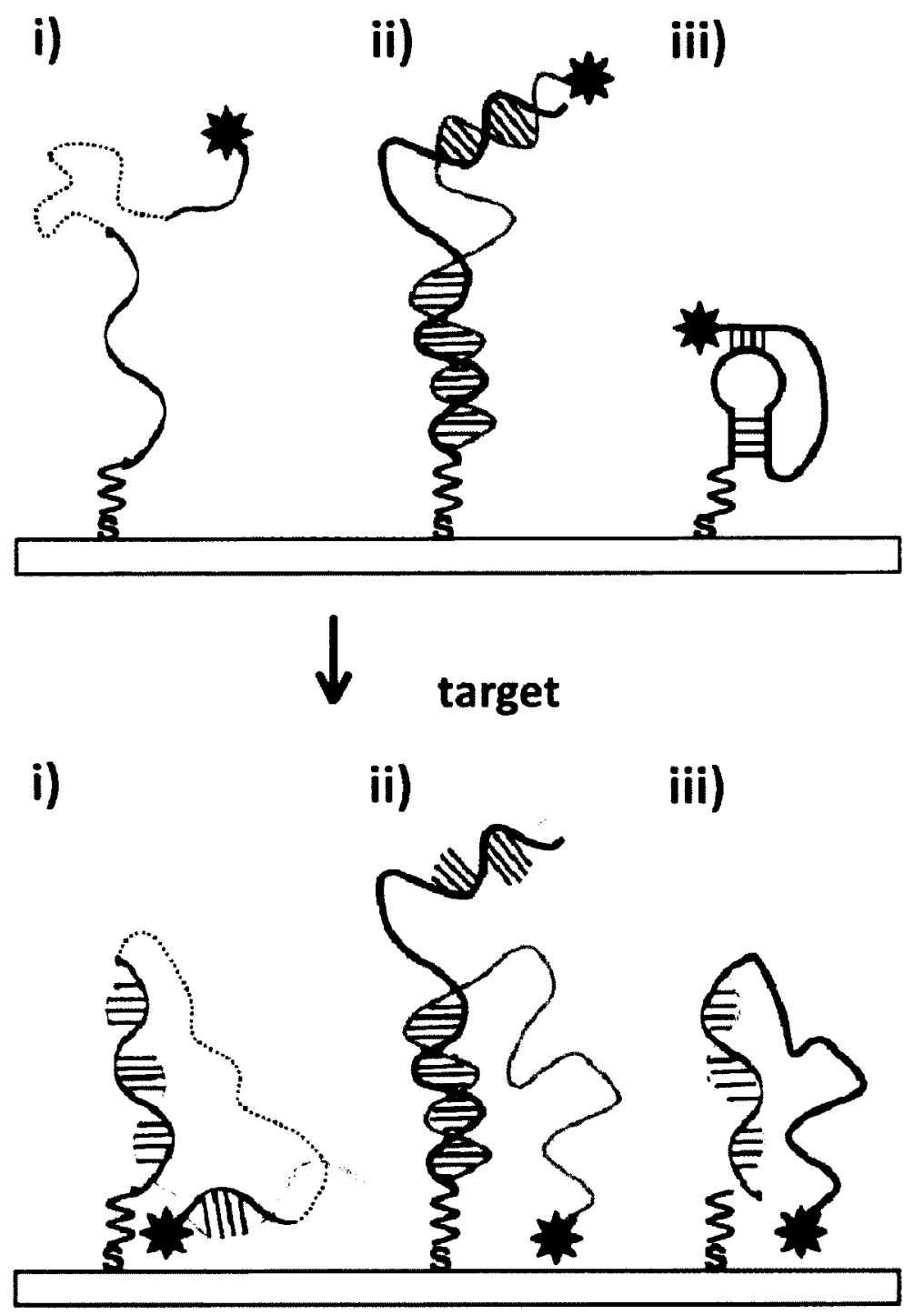

Figure 2-6: Signal-on architectures for electrochemical DNA sensors: i) Sandwich architecture employing capture and probe-modified strands, ii) Target induced strand displacement, iii) Target induced pseudoknot disruption. (adapted from Miranda-Castro et al.) ${ }^{20}$

E-DNA sensors are applicable in a wide range of sample matrices, including cell lysates, blood, and soil extracts ${ }^{79}$. Detection limits in the low picomolar range make EDNA sensors particularly attractive in both clinical and defense settings ${ }^{74}$. They are fully 
reusable, and amenable to integration in microfluidic devices ${ }^{34}$. Several of the design principles and advantages of E-DNA sensors extend directly to electrochemical aptamerbased (E-AB) sensors for a far wider range of analytes ${ }^{34}$.

\subsubsection{Electrocatalysis for mismatch detection}

The term 'electrocatalysis' as coined by Kobozev in 1936 and proliferated by Grubb in the 1960s has traditionally been associated with the acceleration of reactions involving gases related to fuel storage and conversion applications ${ }^{80}$. In the context of biosensing and this project however, electrocatalysis refers to the in situ regeneration of an electroactive probe so that it may be interrogated repeatedly by an electrode ${ }^{81}$. The consumption of the species which regenerates the electroactive probe may be thought of as the catalyzed reaction, with the resulting increase in electron flow providing amplification of the analytical signal. The general reaction scheme, where the probe is restored by species $Z^{65}$ :

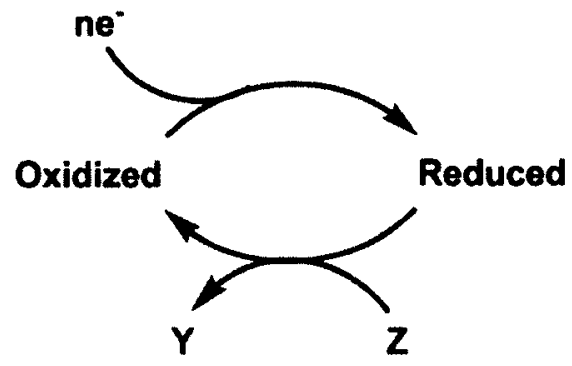

Kelley et al. used an electrocatalytic cycle between methylene blue (MB) and ferricyanide, shown in Figure 2-7, to detect single base pair mismatches in double stranded DNA (dsDNA) $)^{82}$. 
<smiles></smiles>

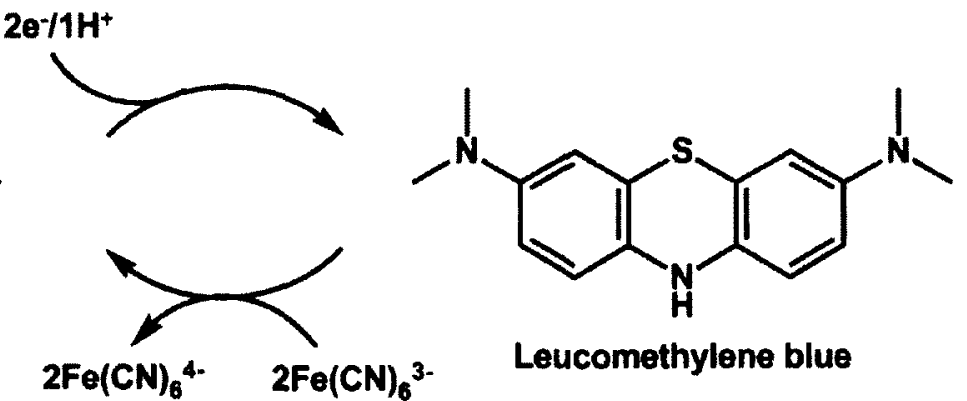

Figure 2-7: Electrocatalytic reduction of ferricyanide by methylene blue. (adapted from Farjami et al.) ${ }^{83}$

Methylene blue was previously speculated to bind via intercalation toward the solvent exposed terminus of the duplexes, with the crowding of duplexes on the electrode preventing a more even distribution along the strand ${ }^{84}$. Indeed, this was exploited in the mismatch detection as charge transport through the duplex was diminished by interruption of $\pi$-stacking ${ }^{84}$. The interruption of charge transport was detectable by both $\mathrm{CV}$ and $\mathrm{CC}$ experiments ${ }^{82}$.
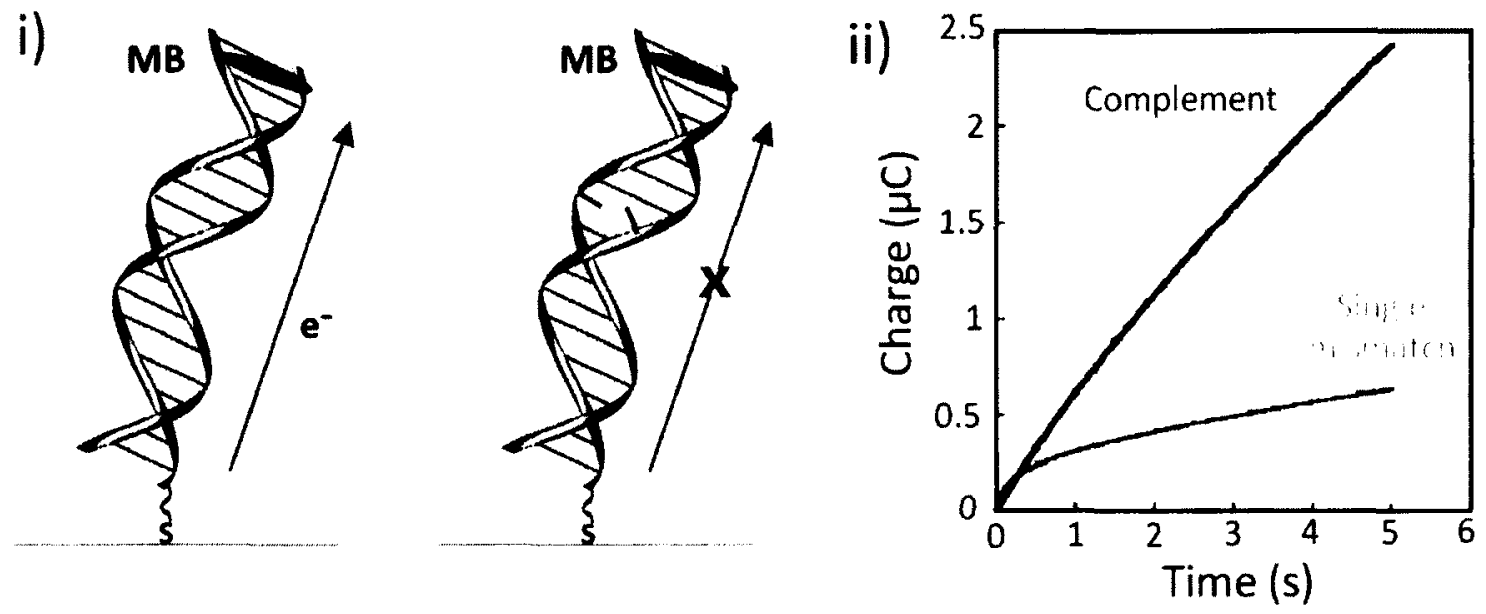

Figure 2-8: i) Interruption of charge transport for detection of a single mismatch, ii) Chronocoulometric detection of this interruption. Data corresponds to an experiment which included electrocatalytic enhancement. (adapted from Boon et al. and Kelley et al.) ${ }^{82,85}$ 
Using an electrocatalytic cycle between intercalated daunomycin and dissolved oxygen in solution, this approach was extended to the detection of protein interactions with immobilized duplex DNA by the same mechanism of charge transport interruption ${ }^{86}$. The goal of this project will be to extend the electrocatalytic enhancement to an aptamerbased sensor.

\subsubsection{Electrochemical aptasensors}

The first generation of aptamer-based sensors were based on optical detection schemes, with sensors based on electrochemical detection not appearing until the early $2000 \mathrm{~s}^{33,58}$. Electrochemical aptasensors developed rapidly however, requiring only four years to equal optical aptasensors in publications per year ${ }^{33}$. This rapid expansion is not surprising given the plethora of advantages associated with electrochemical methods. Most sensors prepared to date are for targets that are simply convenient for proof-ofconcept experiments, such as thrombin or ATP, unfortunately making comparisons to established clinical tests difficult ${ }^{32}$. Nevertheless, the electrochemical aptasensor platform holds great promise. Electrochemical measurements are highly sensitive, robust, and are not confounded by turbid samples ${ }^{52}$. Additionally, electrochemical instrumentation is far cheaper, requires less power, and is the most amenable to miniaturization ${ }^{73}$.

The first electrochemical aptamer based sensor was constructed by immobilizing a methylene blue modified thrombin binding aptamer onto a gold electrode ${ }^{32}$. In the absence of target, the aptamer exists in equilibrium between single stranded and binding competent G-quadruplex states. As thrombin target is introduced, the equilibrium is 
driven toward the G-quadruplex structure. This change significantly alters the ability of the redox-active methylene blue probe to interact with the electrode, causing a reduction in signal ${ }^{87}$. A signal-on version of the sensor quickly followed, where an inhibiting duplex structure is disrupted upon target binding, freeing the methylene blue tag to interact with the electrode ${ }^{35}$. A short non-complementary region between the two duplex components prevents the charge transport utilized in the mismatch sensors of Kelley et $\mathrm{al}^{82}$.

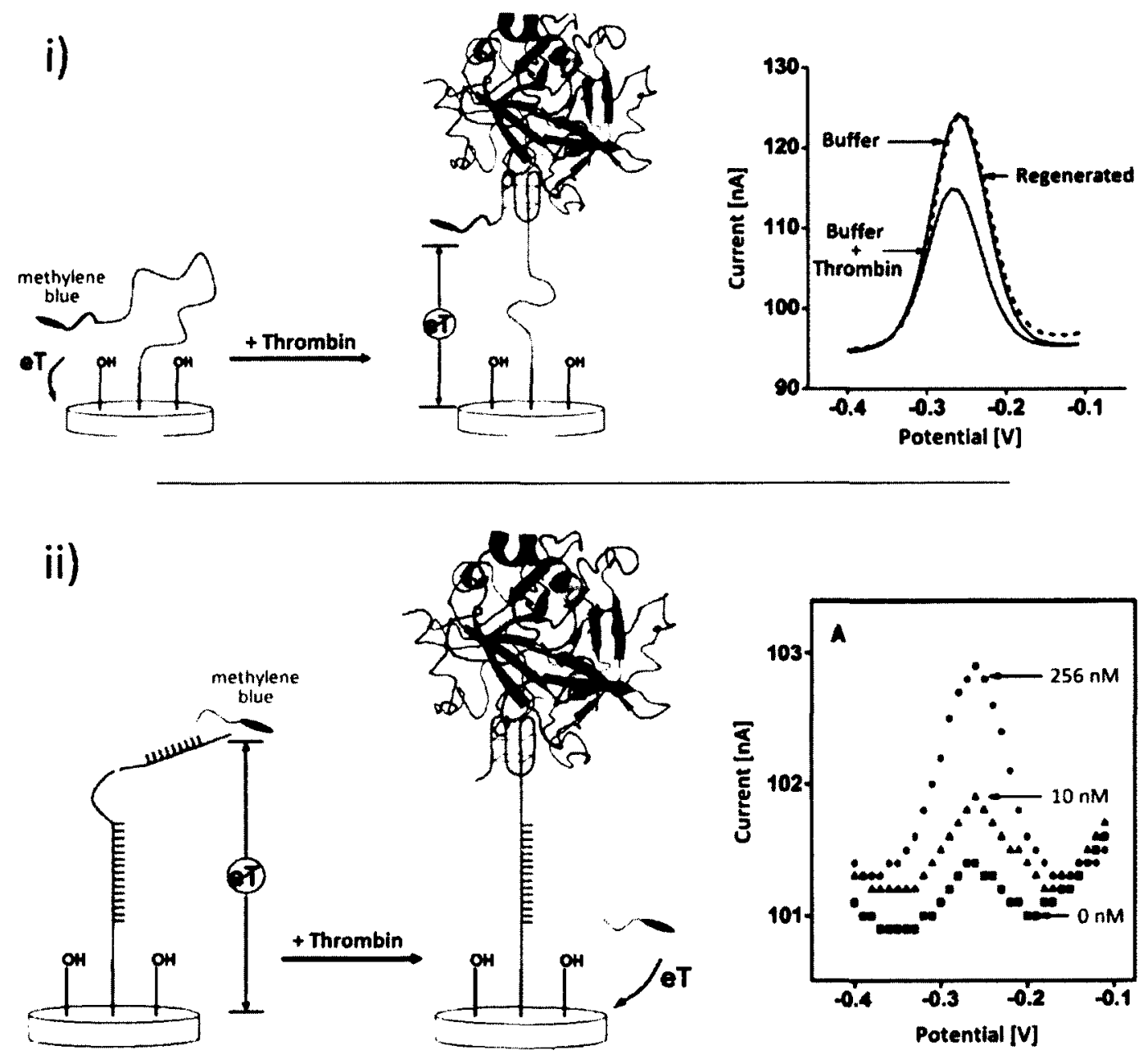

Figure 2-9: Original signal-off (i) and signal-on (ii) electrochemical aptasensor designs proposed by Xiao et al. Signal arises as binding-induced changes in conformational dynamics modify the interaction between redox-active methylene blue and the gold electrode surface. (adapted from Lubin et al.) ${ }^{32}$ 
Sensor constructs where the redox-active probe is not covalently attached, but rather intercalated in a duplex structure or electrostatically bound, are also popular ${ }^{88}$. Aptamer-target binding displaces these probes, with the difference in charge transfer mechanisms as bound probes are displaced providing the analytical signal ${ }^{88}$. Another sensor type, impedimetric aptasensors, exploit binding induced changes in resistance to electron-transfer reactions at aptamer modified electrodes ${ }^{89}$. Wang and coworkers developed a sensor of this type for lysozyme, presented in Figure $2-10^{90}$. The unbound lysozyme aptamer, as a polyanion, provides greater resistance to the anionic ferrocyanide/ferricyanide redox couple. Lysozyme binding at the correct $\mathrm{pH}$ provides charge compensation and decreases electron transfer resistance ${ }^{89}$. Radi et al. prepared an impedimetric aptasensor for thrombin utilizing a gold electrode and the same redox couple $^{91}$. Impedimetric aptasensors carry the advantage of requiring only the chemical modifications needed for immobilization, covalent attachment of a probe moiety is not necessary ${ }^{89}$.
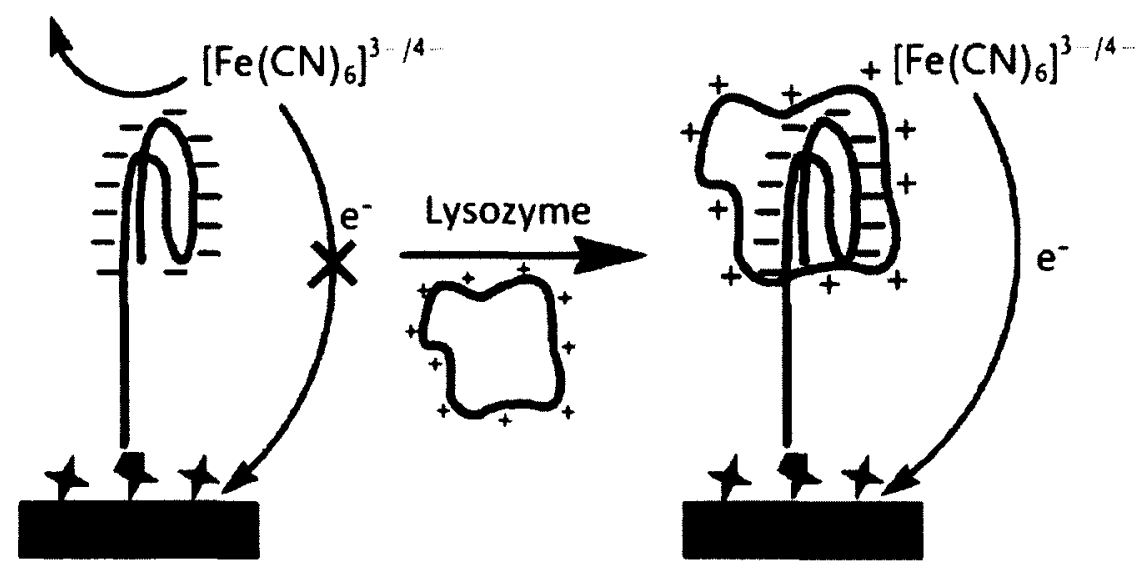

Figure 2-10: An impedimetric aptasensor for lysozyme. Target-binding at the appropriate $\mathrm{pH}$ provides the charge compensation to allow an anionic redox-active probe to approach the electrode. (adapted from Willner et al.) ${ }^{89}$ 
As aptamers may be selected to bind to different epitopes on a target molecule, many sandwich-type sensor designs have also emerged ${ }^{18}$. While currently limited to the very small subset of aptamer targets for which multiple aptamers have been selected, these designs can increase the already impressive sensitivity and selectivity of electrochemical aptasensors ${ }^{92}$. In addition, catalytic labels such as enzymes or inorganic nanoparticles have been used for signal amplification ${ }^{89}$. An example of both concepts can be seen in an aptasensor prepared by Polsky et al., shown in Figure $2-11^{93}$. Monitoring the catalytic reduction of hydrogen peroxide by the platinum nanoparticles enhanced the limit of detection by almost two orders of magnitude ${ }^{89}$.

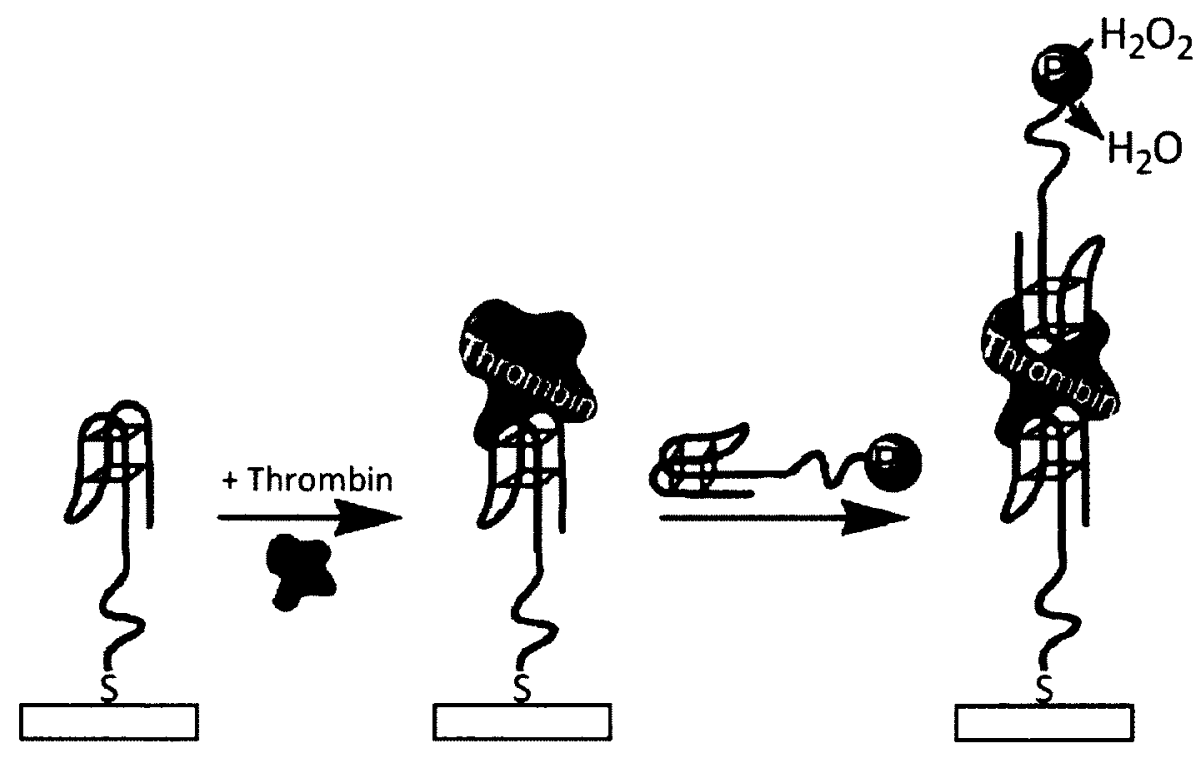

Figure 2-11: An electrochemical aptasensor for thrombin incorporating both the sandwich architecture and catalytic labeling concepts. (adapted from Willer et al.) ${ }^{89}$ 


\subsubsection{Project objectives}

The objective of this project will be to combine elements of previously reported works using electrocatalysis for mismatch detection with an established folding-based aptasensor in a novel approach to improve aptasensor performance. First, synthesis and purification of the methylene blue labeled aptamer required to construct an aptasensor for thrombin will be described. After confirming its consistency with similar sensors reported in the literature, the sensor produced will be deployed in the presence of potassium ferricyanide substrate to investigate possible electrocatalytic enhancement of sensor performance.

\subsection{Experimental}

\subsubsection{Materials}

All buffers were prepared with Millipore Milli-Q deionized water at $18 \mathrm{M} \Omega$. Salts used in the preparation of buffers were technical grade or higher. Phophoramidites and modifiers, modified support columns, activator, deblock, capping, and oxidizing reagents were obtained from Glen Research. Standard support columns and acetonitrile were purchased from BioAutomation. Ultra High Purity 5.0 argon was purchased from Praxair. HPLC grade acetonitrile was supplied by Caledon. All other chemicals and solvents were of reagent grade or higher, and used as received. 


\subsubsection{Synthesis of methylene blue-modified thrombin aptamer (MB-DNA)}

\subsubsection{Oligonucleotide synthesis}

Oligonucleotides were synthesized via standard phosphoramidite chemistry on a BioAutomation Corporation MerMade 6 DNA Synthesizer (Plano, TX). Sequences were produced at the $1 \mu \mathrm{mol}$ scale on $500 \AA$ pore size columns. No changes to synthesizer manufacturer conditions were made, with the exception of a 60 second increase in the coupling time of 5'-modifiers. The following sequences were produced:

Thrombin aptamer: $\mathrm{HO}-\left(\mathrm{CH}_{2}\right)_{6}$-S-S- $\left(\mathrm{CH}_{2}\right)_{6}-5$ '-TAA GTT CAT CTC CCC GGT TGG TGT GGT TGG T-3'- $\mathrm{CH}_{2}-\mathrm{CH}\left(\mathrm{CH}_{2} \mathrm{OH}\right)-\left(\mathrm{CH}_{2}\right)_{4}-\mathrm{NH}_{2}$

\section{SH-DNA: $\mathrm{HO}-\left(\mathrm{CH}_{2}\right)_{6}-\mathrm{S}-\mathrm{S}-\left(\mathrm{CH}_{2}\right)_{6}-5^{\prime}-\mathrm{ACC}$ TGG GGG AGT ATT GCG GAG GAA GGT-3'}

Following the automated portion of the synthesis, the columns were removed, and placed on a BioAutomation cleaving block. Columns were treated with $1 \mathrm{~mL}$ of $10 \%$ diethylamine in anhydrous acetonitrile, washed with two column volumes of acetonitrile, and dried with argon. CPG support beads were transferred to microcentrifuge tubes, and $1 \mathrm{~mL}$ of aqueous ammonia was added. The tubes were safety capped and left overnight at $37^{\circ} \mathrm{C}$. CPG beads were separated via filtration with $0.22 \mu \mathrm{m}$ nylon microcentrifuge filter tubes (Spin-X, Corning). Filtrates were dried on a Savant AES2010 SpeedVac system. Crude oligonucleotides were resuspended in conjugation buffer $(0.1 \mathrm{M}$ sodium phosphate, $\mathrm{pH} 8.0$ ) and characterized with a Cary Bio $300 \mathrm{UV}-\mathrm{V}$ is spectrophotometer. 


\subsubsection{Conjugation of methylene blue to DNA}

Dicarboxymethylene blue NHS ester (DCMB-SE: 3,7-bis(N-(3-carboxypropyl)$\mathrm{N}$-methylamino)-phenothiazin-5-ium perchlorate, succinimidyl ester) was purchased from emp Biotech (Berlin). DCMB-SE $(0.3 \mathrm{mg}, 400 \mathrm{nmol})$ was dissolved in $200 \mu \mathrm{L}$ of N,N-dimethylformamide (DMF), and combined with crude oligonucleotides $(1.1 \mathrm{mg}$, $100 \mathrm{nmol})$ in $100 \mu \mathrm{L}$ conjugation buffer in an amber microcentrifuge tube. The tube was shaken for 1 hour then left to sit in a dark drawer overnight. The reaction mixture was then dried by SpeedVac.<smiles>CC[C@@H](CCCN(C)c1ccc2nc3ccc(N(C)CCCC(=O)ON4C(=O)CCC4=O)cc3[s+]c2c1)ON1C(=O)CCC1=O</smiles><smiles></smiles>

Figure 2-12: Active ester conjugation of DCMB-SE to 3'-NH $\mathrm{NH}_{2}$ modified DNA. Hydrolysis of the second succinimidyl ester occurs during work up. 


\subsubsection{Purification of MB-DNA}

Ethanol precipitation was first used to separate non-covalently bound methylene blue from DNA. The crude conjugation reaction products were resuspended in $100 \mu \mathrm{L}$ of deionized water in a microcentrifuge tube. Ethanol precipitation was performed by adding 10 volumes of anhydrous ethanol, and 0.5 volumes of $3 \mathrm{M} \mathrm{NaCl}$. The mixture was briefly vortexed and then placed on dry ice for one hour. The tube was centrifuged at maximum speed for 5 minutes at $-9^{\circ} \mathrm{C}$, returned to the dry ice for an additional 5 minutes, then centrifuged again. The supernatant was removed slowly by pipette.

Ethanol precipitation was repeated until the supernatant appeared colourless, requiring between 6-12 rounds. Supernatants were dried via Speed Vac, and the resulting solids were dissolved in deionized water for characterization by UV-Vis spectrophotometry. The DNA pellet from the final ethanol precipitation step was dissolved in $500 \mu \mathrm{L}$ of deionized water, and desalted with an Amicon Ultra centrifugal filter featuring a 3kDa cutoff membrane (Millipore).

RP-HPLC was then used to isolate methylene blue-labeled from unlabeled DNA. Isolation was performed on a Phenomenex Clarity Oligo-RP C18 column (50mm length $\mathrm{x}$ $10 \mathrm{~mm}$ internal diameter, $3 \mu \mathrm{m}$ particle size), with the mobile phase program described in Table 2-1. 
Table 2-1: Reverse phase HPLC program for isolation of MB-DNA.

\begin{tabular}{|l|l|l|l|l|}
\hline Time $(\mathrm{min})$ & $\begin{array}{l}\text { Fraction of Solvent A: } \\
50 \mathrm{mM} \text { TEAA pre-mixed } \\
\text { with 5\% acetonitrile }\end{array}$ & $\begin{array}{l}\text { Fraction of } \\
\text { Solvent B: } \\
\text { acetonitrile }\end{array}$ & $\begin{array}{l}\text { Flow rate } \\
(\mathrm{mL} / \mathrm{min})\end{array}$ & $\begin{array}{l}\text { Column } \\
\text { temperature } \\
\left({ }^{\circ} \mathrm{C}\right)\end{array}$ \\
\hline 0 & $5 \%$ & $95 \%$ & 2 & 25 \\
\hline 30 & $85 \%$ & $15 \%$ & 2 & 25 \\
\hline 40 & $60 \%$ & $40 \%$ & 2 & 25 \\
\hline
\end{tabular}

RP-HPLC fractions were collected in $15 \mathrm{~mL}$ polyethylene terephthalate (PET) tubes, frozen by immersing the tubes in liquid nitrogen, and lyophilized overnight. Fractions were resuspended in deionized water for characterization by UV-Vis spectrophotometry. Dry samples containing $1 \mathrm{nmol}$ each were shipped to Novatia LLC for ESI-MS.

\subsubsection{Ellman's reaction}

A small aliquot of purified MB-DNA $(0.5 \mathrm{nmol})$ was mixed with a 100 -fold excess of TCEP (tris(2-carboxyethyl)phosphine hydrochloride) in $10 \mathrm{mM}$ tris(hydroxymethyl)aminomethane (referred to hereafter as tris) buffer to reduce disulfides. After desalting, DTNB (5,5'-dithiobis-2-nitrobenzoic acid) in $10 \mathrm{mM}$ tris buffer was added in a 1:1 molar ratio with MB-DNA. The reaction was allowed to proceed for 1 hour, and the products were investigated by UV-Vis. The products were then desalted, dried by SpeedVac, and shipped to Novaia LLC for ESI-MS analysis. 
<smiles>CC[C-]Sc1ccc([N+](=O)[O-])c(C(=O)[O-])c1</smiles>

Figure 2-13: Ellman's reaction. Absorbance of the 5-thio-2-nitrobenzoic acid anion produced $\left(\mathrm{TNB}^{2-}, \varepsilon_{412 \mathrm{~nm}}=14150 \mathrm{M}^{-1} \mathrm{~cm}^{-1}\right)$ may be used for the quantification of thiol modified compounds. (adapted from Riddles et al.) ${ }^{94}$

\subsubsection{Other approaches to MB-DNA synthesis and purification}

While the preceding sections outlined the eventually successful preparation of MB-DNA, it should be noted that other reaction solvents, molar ratios and purification routes were attempted. These will be described here in brief for completeness.

Alternate solvents - 0.1M 4-(2-hydroxyethyl)-1-piperazineethanesulfonic acid (HEPES) pH 8.3 was used as the conjugation buffer and anhydrous dimethyl sulfoxide (DMSO) was used to solubilize DCMB-SE in other reaction attempts. Molar ratios of DCMB-SE to DNA ranging from $1: 1,2: 1$, to $4: 1$ were evaluated. For RP-HPLC purification, $50 \mathrm{mM}$ ammonium acetate buffer was initially used as Solvent A.

Poly(acrylamide) gel electrophoresis (PAGE) - A 19\% PAGE gel was prepared to analyze products of the MB-DNA conjugation reaction. The monomer solution was prepared by combining $37.5 \mathrm{~mL}$ of acrylamide stock $\left(190 \mathrm{~g}\right.$ acrylamide and $10 \mathrm{~g} \mathrm{~N}, \mathrm{~N}^{\prime}-$ methylenebisacrylamide dissolved to $500 \mathrm{~mL}$ with deionized water and filtered), $15 \mathrm{~mL}$ of 5x TBE buffer (45mM tris, $45 \mathrm{mM}$ boric acid, $1 \mathrm{mM}$ EDTA), and $31.5 \mathrm{~g}$ urea. Components were dissolved by heating to $37^{\circ} \mathrm{C}$ with magnetic stirring. The solution was passed through Whatman No. 1 filter paper and cooled to room temperature. With gentle 
swirling, $450 \mu \mathrm{L}$ of $10 \%$ ammonium persulfate in deionized water and $35 \mu \mathrm{L}$ of $\mathrm{N}, \mathrm{N}, \mathrm{N}^{\prime}, \mathrm{N}^{\prime}$-tetramethylethylenediamine (TEMED) were added. The solution was slowly poured into a pre-assembled glass plate assembly and a comb was inserted to mold the injection wells. After a polymerization time of one hour, the comb was removed, and the wells were rinsed with deionized water. The glass plate assembly was transferred to an electrophoresis tank, and the upper and lower chambers were filled with 1x TBE. The gel was equilibrated for 30 minutes at a constant current of $25 \mathrm{~mA}$. Conjugation reaction products $\left(2 \mathrm{OD}_{260 \mathrm{~nm}}\right.$ per lane) in no more than $10 \mu \mathrm{L}$ were combined with equal volumes of formamide and heated to $55^{\circ} \mathrm{C}$ for 5 minutes before loading. Gels were run with constant current at $25 \mathrm{~mA}$, stopping when a fluorescently tagged 18 -mer tracer moved $2 / 3$ of the way down the gel. An Alpha Innotech Alphalmager EC instrument was used to characterize the gel. Bands of interest were excised and eluted by incubating the crushed gel in deionized water overnight at $37^{\circ} \mathrm{C}$.

Amicon Ultra desalting columns - Crude conjugation products resuspended in deionized water were filtered with $3 \mathrm{kDa}$ cutoff Amicon Ultra centrifugal filter columns. The retentate was repeatedly washed with deionized water in one trial, and with $140 \mathrm{mM}$ $\mathrm{NaCl}$ in another. The filtrates as well as the final retentate were analyzed by UV-Vis.

Clarity QSP cartridges - Clarity QSP cartridges were used for purification of the modified thrombin aptamer described in Section 2.2.2.1 prior to the conjugation reaction. QSP cartridges and compatible vacuum manifold were obtained from Phenomenex. An important deviation from the manufacturer procedure was the substitution of $0.1 \mathrm{M}$ tris pH 7.4 buffer for Phenomenex-branded 'DNA loading buffer'. For each step described in Table 2-2, the entire volume was allowed to pass through the cartridge. 
Table 2-2: Clarity QSP protocol for purification of modified thrombin aptamer.

\begin{tabular}{|l|l|l|}
\hline \multicolumn{1}{|c|}{ Step } & Solvent/Reagent Volume & \multicolumn{1}{|c|}{ Flow Rate } \\
\hline Conditioning & $2 \times 1.5 \mathrm{~mL}$ methanol & 2 drops $/ \mathrm{s}$ \\
\hline Loading & $\begin{array}{l}1 \mathrm{~mL} \text { crude thrombin aptamer } \\
\text { in aqueous ammonia and } \\
1 \mathrm{~mL} 0.1 \mathrm{M} \text { tris } \mathrm{pH} 7.4\end{array}$ & $1 \mathrm{drop} / \mathrm{s}$ \\
\hline Wash & $\begin{array}{l}0.75 \mathrm{~mL} 0.1 \mathrm{M} \text { tris } \mathrm{pH} 7.4 \\
0.75 \mathrm{~mL} \text { deionized water }\end{array}$ & 1 drop/s \\
\hline Detritylation & $\begin{array}{l}1.5 \mathrm{~mL} 2 \% \text { dichloroacetic } \\
\text { acid in deionized water }\end{array}$ & 2 drops $/ \mathrm{s}$ \\
\hline Rinsing & $2 \times 1.0 \mathrm{~mL}$ deionized water & $2 \mathrm{drops} / \mathrm{s}$ \\
\hline Elution & $\begin{array}{l}2 \mathrm{~mL} 0.1 \mathrm{M} \text { tris } \mathrm{pH} 7.4 / 20 \% \\
\text { acetonitrile }\end{array}$ & $1 \mathrm{drop} / \mathrm{s}$ \\
\hline
\end{tabular}

Bio Spin 6 columns - Bio Spin 6 gel purification columns were obtained from Bio Rad. The columns were shaken to resuspend the gel after shipping, and the packing buffer was allowed to drain by gravity. An aliquot of crude MB-DNA reaction products $\left(\sim 50 \mathrm{OD}_{260 \mathrm{~nm}}\right)$ were loaded onto the center of the gel bed, and the column was centrifuged at $1000 \mathrm{~g}$ for 4 minutes. The collected product was analyzed by UV-Vis. 


\subsubsection{Preparation of MB-DNA modified electrodes}

\subsubsection{Preparation of gold electrodes}

Gold working electrodes ( $\mathrm{CH}$ Instruments) were polished with fine alumina particles on velvet microcloth pads obtained from a standard electrode polishing kit (BASi). First, the pads were wetted with deionized water and spotted with several drops of the alumina suspension. The gold electrodes were polished by tracing figure-eight and z-patterns in the pad with firm pressure for 5 minutes. The electrodes were rinsed, and sonicated in ethanol for 15 minutes. Individually, the electrodes were immersed in cells containing $10 \mathrm{~mL}$ of $1 \mathrm{M} \mathrm{H}_{2} \mathrm{SO}_{4}$.

The cell was completed with an $\mathrm{Ag} / \mathrm{AgCl}$ reference electrode and platinum wire auxiliary electrode, and connected to a $\mathrm{CHI} 660 \mathrm{C}$ potentiostat (CH Instruments). The cell was deoxygenated by bubbling argon for 10 minutes. The gold electrodes were then electrochemically cleaned by running the cyclic voltammetry experiment described in Table 2-3. The entire cleaning procedure was repeated until the final voltammogram resembled that presented in Figure 2-14. On average, two repetitions of the procedure were required. Finally, the electrodes were rinsed thoroughly with deionized water and dried with argon. 
Table 2-3: Cyclic voltammetry parameters for electrochemical cleaning of gold electrodes in sulfuric acid.

\begin{tabular}{|l|l|}
\hline Initial E & $0.00 \mathrm{~V}$ \\
\hline High E & $1.65 \mathrm{~V}$ \\
\hline Low E & $-0.25 \mathrm{~V}$ \\
\hline Initial scan polarity & Negative \\
\hline Scan rate & $0.2 \mathrm{~V} / \mathrm{s}$ \\
\hline Sweep segments & 21 \\
\hline Sample interval & $0.001 \mathrm{~V}$ \\
\hline Quiet time & $2.0 \mathrm{~s}$ \\
\hline
\end{tabular}

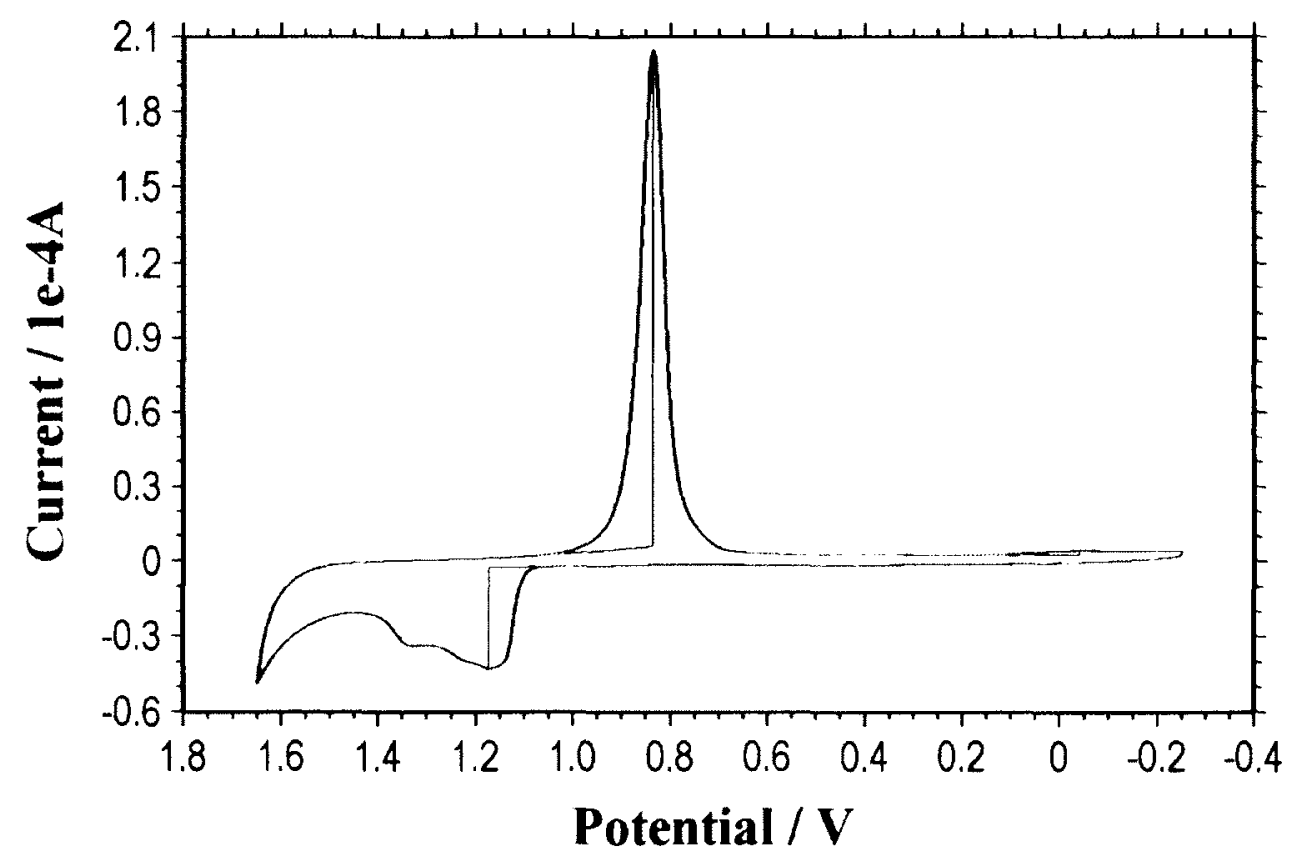

Figure 2-14: Cyclic voltammogram of a clean gold working electrode. 


\subsubsection{Deposition of MB-DNA and SH-DNA on gold electrodes}

Purified MB-DNA and SH-DNA were each dissolved in $10 \mathrm{mM}$ tris buffer with $1 \mathrm{mM}$ EDTA (pH 8.0) to a concentration of $200 \mu \mathrm{M}$. A $10 \mathrm{mM}$ solution of tris(2carboxyethyle)phosphine hydrochloride (TCEP) was prepared in the same buffer. It was critical that the buffer was deoxygenated via bubbling argon before preparing the TCEP solution. For each electrode to be prepared, $2 \mu \mathrm{L}$ of DNA solution and $4 \mu \mathrm{L}$ of TCEP solution were combined in a $2.0 \mathrm{~mL}$ microcentrifuge tube, and mixed via pipette.

The blue colour of methylene blue was used to indicate the completeness of this reaction. If the resulting solution had not become colourless within one hour, an additional $4 \mu \mathrm{L}$ of TCEP solution was added and the solution was incubated for another 30 minutes. SH-DNA depositions were assumed to be progressing at the same rate as a concurrent MB-DNA sample. The reduced DNA solutions were diluted to $100 \mu \mathrm{L}$ with deoxygenated $10 \mathrm{mM}$ tris, $1 \mathrm{M} \mathrm{NaCl}, 1 \mathrm{mM}$ EDTA (pH 7.6) buffer.

Freshly cleaned gold electrodes were immersed in the microcentrifuge tubes. The tubes were sealed with laboratory film and placed in a dark cupboard for 48 hours.

\subsubsection{Treatment with 6-mercapto-1-hexanol}

Electrodes were removed from the DNA solutions and rinsed with deionized water for 10 seconds. Excess water was wicked away and the electrodes were immersed in clean $2.0 \mathrm{~mL}$ microcentrifuge tubes containing $100 \mu \mathrm{L}$ of $1 \mathrm{mM} \mathrm{6-mercapto-1-hexanol}$ $(\mathrm{MCH})$ in ethanol. The tubes were sealed with laboratory film and returned to the dark 
cupboard for 24 hours. Electrodes were rinsed with deionized water for 1 minute, then either set directly into an electrochemical cell, or immersed in storage phosphate buffered saline (s-PBS, $10 \mathrm{mM}$ phosphate, $1 \mathrm{M} \mathrm{NaCl}, 1 \mathrm{mM} \mathrm{Mg}^{2+}, \mathrm{pH}$ 7.2) and sealed with laboratory film for storage at $4^{\circ} \mathrm{C}$. If stored, electrodes were rinsed with deionized water for 1 minute prior to use.

\subsubsection{Electrochemical characterization of modified electrodes}

All electrochemical measurements on DNA modified gold working electrodes were made in cells completed with an $\mathrm{Ag} / \mathrm{AgCl}$ reference electrode and platinum wire auxiliary electrode. Four buffer solutions were used in various experiments:

PBS: $5 \mathrm{mM}$ phosphate, $50 \mathrm{mM} \mathrm{NaCl}, \mathrm{pH} 7$

Tris: $10 \mathrm{mM}$ tris(hydroxymethyl)aminomethane, $\mathrm{pH} 7.4$

Thrombin-aptamer binding buffer (TBB): $140 \mathrm{mM} \mathrm{NaCl}, 100 \mathrm{mM}$ tris, $20 \mathrm{mM}$ $\mathrm{MgCl}_{2}, 20 \mathrm{mM} \mathrm{KCl}, \mathrm{pH} 7.4$

Thrombin-aptamer binding buffer mimic, at the same ionic strength, but without potassium or magnesium (NoKTBB): $220 \mathrm{mM} \mathrm{NaCl}, 100 \mathrm{mM}$ tris, $\mathrm{pH} 7.4$

Cells were deoxygenated with bubbling argon for 10 minutes prior to measurement. Cyclic voltammetry, square wave voltammetry, and chronocoulometry were performed with the parameters indicated in Tables 2-4 through 2-6 respectively. MB-DNA and SHDNA modified surfaces were characterized in each buffer alone, and in each buffer with varying concentrations of potassium ferricyanide. 
Table 2-4: Cyclic voltammetry parameters used for electrochemical characterization of DNA modified gold electrodes.

\begin{tabular}{|l|l|}
\hline Initial E & $0.0 \mathrm{~V}$ \\
\hline High E & $0.5 \mathrm{~V}$ \\
\hline Low E & $-0.5 \mathrm{~V}$ \\
\hline Initial scan polarity & Negative \\
\hline Scan rate & $0.1 \mathrm{~V} / \mathrm{s}$ \\
\hline Sweep segments & 10 \\
\hline Sample interval & $0.001 \mathrm{~V}$ \\
\hline Quiet time & $2.0 \mathrm{~s}$ \\
\hline
\end{tabular}

Table 2-5: Square wave voltammetry parameters used for electrochemical characterization of DNA modified gold electrodes.

\begin{tabular}{|l|l|}
\hline Initial E & $0.0 \mathrm{~V}$ \\
\hline Final E & $-0.5 \mathrm{~V}$ \\
\hline Incremental E & $0.001 \mathrm{~V}$ \\
\hline Amplitude & $0.025 \mathrm{~V}$ \\
\hline Frequency & $60 \mathrm{~Hz}$ \\
\hline Quiet time & $2.0 \mathrm{~s}$ \\
\hline
\end{tabular}


Table 2-6: Chronocoulometry parameters used for electrochemical characterization of DNA modified gold electrodes.

\begin{tabular}{|l|l|}
\hline Initial E & $0 \mathrm{~V}$ \\
\hline Final E & $\begin{array}{l}\text { Voltage where maximum current observed } \\
\text { on corresponding cyclic voltammetry } \\
\text { experiment. }\end{array}$ \\
\hline Number of steps & 2 \\
\hline Pulse width & $5.0 \mathrm{~s}$ \\
\hline Sample interval & $0.00025 \mathrm{~s}$ \\
\hline Quiet time & $2.0 \mathrm{~s}$ \\
\hline
\end{tabular}

Surface coverage of electrodes modified with SH-DNA was evaluated by monitoring the signal of electrostatically bound ruthenium(III) hexammine ${ }^{95}$. An SHDNA modified electrode was incubated in a $10 \mathrm{~mL}$ electrochemical cell containing $10 \mu \mathrm{M}$ $\left[\mathrm{Ru}\left(\mathrm{NH}_{2}\right)_{6}\right]^{3+}$ in $10 \mathrm{mM}$ tris $\mathrm{pH} 7.4$ for 15 minutes. The cell was completed with an $\mathrm{Ag} / \mathrm{AgCl}$ reference and platinum wire auxilliary electrode. Cyclic voltammetry was performed from $+0.15 \mathrm{~V}$ to $-0.5 \mathrm{~V}$ at a scan rate of $0.5 \mathrm{~V} / \mathrm{s}$, with the remaining parameters identical to those in Table 2-4.

\subsubsection{Sensor operation}

Thrombin sensor experiments were conducted with human $\alpha$-thrombin obtained from Haematologic Technologies Inc. Thrombin aliquots were transferred directly to the electrochemical cell and mixed with a pipette for 30 seconds. Thrombin incubation time 
with the MB-DNA modified surfaces was optimized by characterizing surfaces at five minute intervals for three hours by square wave voltammetry. Calibration curves were generated in PBS buffer with $0.6 \mathrm{mM} \mathrm{KCl}$, and PBS buffer with $0.2 \mathrm{mM} \mathrm{K}_{3} \mathrm{Fe}(\mathrm{CN})_{6}$ added. Single MB-DNA modified electrodes were used for the preparation of each curve. Between experiments at each thrombin concentration, the working electrode was rinsed with deionized water for one minute. A one minute soak in $8 \mathrm{M}$ guanidine hydrochloride was evaluated for sensor regeneration.

\subsection{Results and Discussion}

\subsubsection{Preparation of methylene blue-labeled thrombin aptamer (MB-DNA)}

\subsubsection{Oligonucleotide synthesis}

The 15-mer Bock thrombin binding sequence, with an additional 15 nucleotide linker sequence was synthesized by standard phosphoramidite chemistry without major difficulties. The selection of this particular sequence and linker was based on the work done by Xiao et al. for their signal-off detection of thrombin, shown in Figure 2-9 (i) ${ }^{87}$. The first major component of the project was to develop the protocol for synthesis of the dually modified DNA. This could later be extended to aptamer sequences for other analytes of interest.

The sequences described in Section 2.2.2.1 are deliberately shown without protecting groups on the modifiers, specifically the DMT groups at the thiol modified 5'- 
terminus and fluorenylmethyloxycarbonyl (fmoc) at the amino modified 3 '-terminus. The fmoc group could be removed in a separate step by treatment of the synthesis columns with $20 \%$ pyrimidine in DMF. Subsequent monitoring of the eluate at $301 \mathrm{~nm}\left(\varepsilon_{301 \mathrm{~nm}}=\right.$ $7800 \mathrm{M}^{-1} \mathrm{~cm}^{-1}$ ) as per vendor instructions would allow an estimation of the amount of amine available for conjugation. However in the absence of this step, the fmoc group would simply be removed by aqueous ammonia concurrently with the other base-labile protecting groups ${ }^{96}$.

For purification of oligonucleotides, trityl protecting groups at the 5'-terminus are commonly used as hydrophobic handles for RP-HPLC ${ }^{48}$. Since trityl protecting groups are acid labile, current protocols recommend adding a small amount of non-volatile base and conducting in vacuo concentrations at room temperature to minimize selfdetritylation ${ }^{97}$. Regardless of the desalting procedure, tris buffer used for this purpose was found to later quench the conjugation reaction with DCMB-SE. Therefore, concentration steps were simply carried out at room temperature. Evidence for trityl retention despite the absence of non-volatile base will be presented later.

\subsubsection{Synthesis and purification of MB-DNA}

To prepare the electrochemically active thrombin aptamer, methylene blue was conjugated to DNA by active ester coupling in a manner similar to that reported by Schubert et $\mathrm{a}^{98}$. Specifically, a succinimidyl ester derivative of methylene blue was obtained and used for attachment to amino modified DNA. Isolation of the desired methylene blue-thrombin aptamer conjugate (MB-DNA) was performed in two steps. 
First, excess methylene blue was removed by exploiting its solubility in ethanol ${ }^{99}$. When performing repeated ethanol precipitations, non-covalent methylene blue would partition to the ethanol, forsaking electrostatic interaction with the DNA backbone. The ethanol phases from each round of precipitation were analyzed for methylene blue content by UV-Vis, with the results shown in Figure 2-15. Ethanol precipitation was carried out until the ethanol phase appeared colourless.

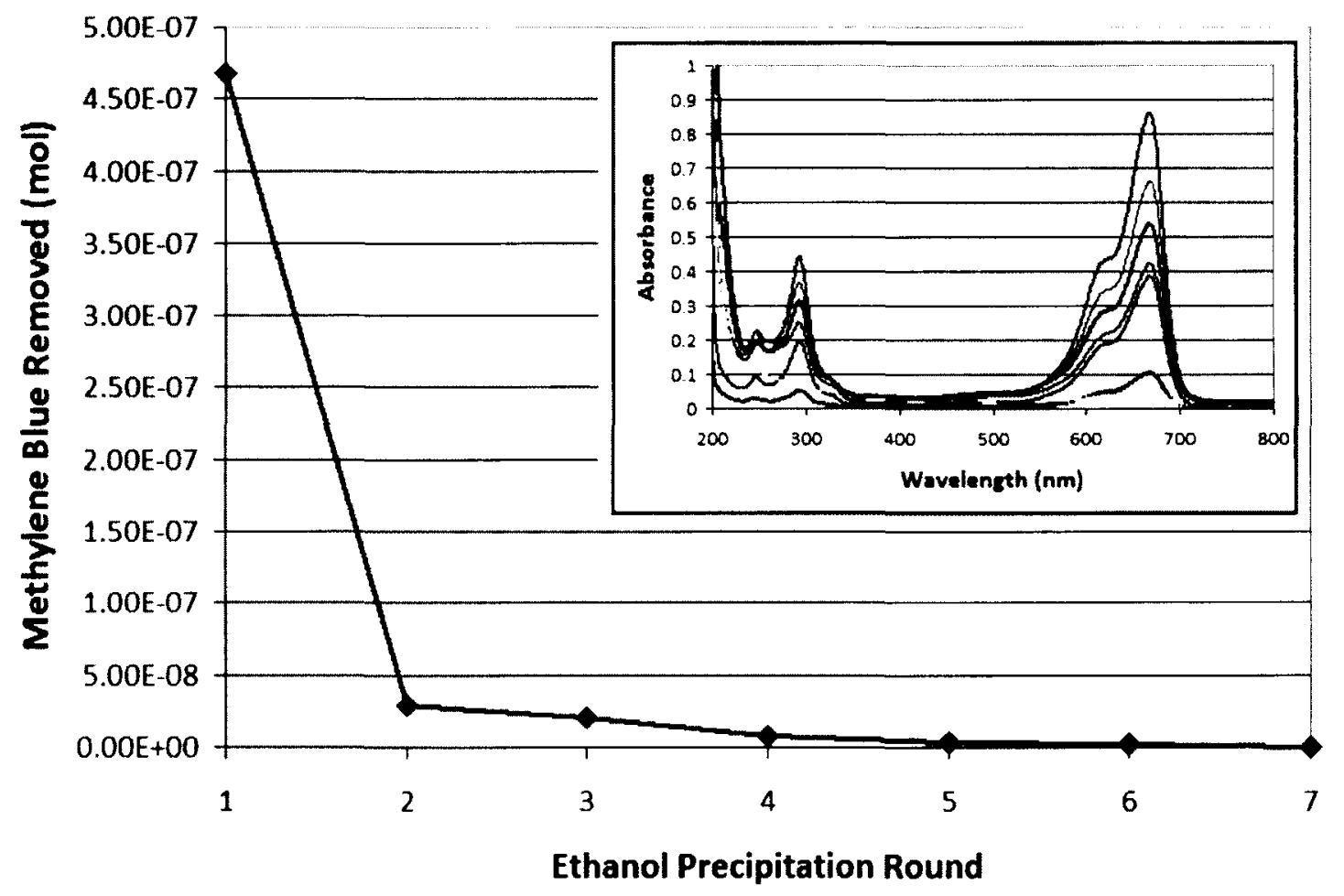

Figure 2-15: Moles of methylene blue removed vs. round of ethanol precipitation, quantified by absorbance at $668 \mathrm{~nm}$. Inset: UV-Visible spectra.

With non-covalent methylene blue removed, RP-HPLC was then used for the separation of methylene blue-labeled from unlabeled DNA. The increased hydrophobic 
character of triethylammonium acetate buffer in the mobile phase improved retention of DNA in comparison to ammonium acetate ${ }^{97}$.

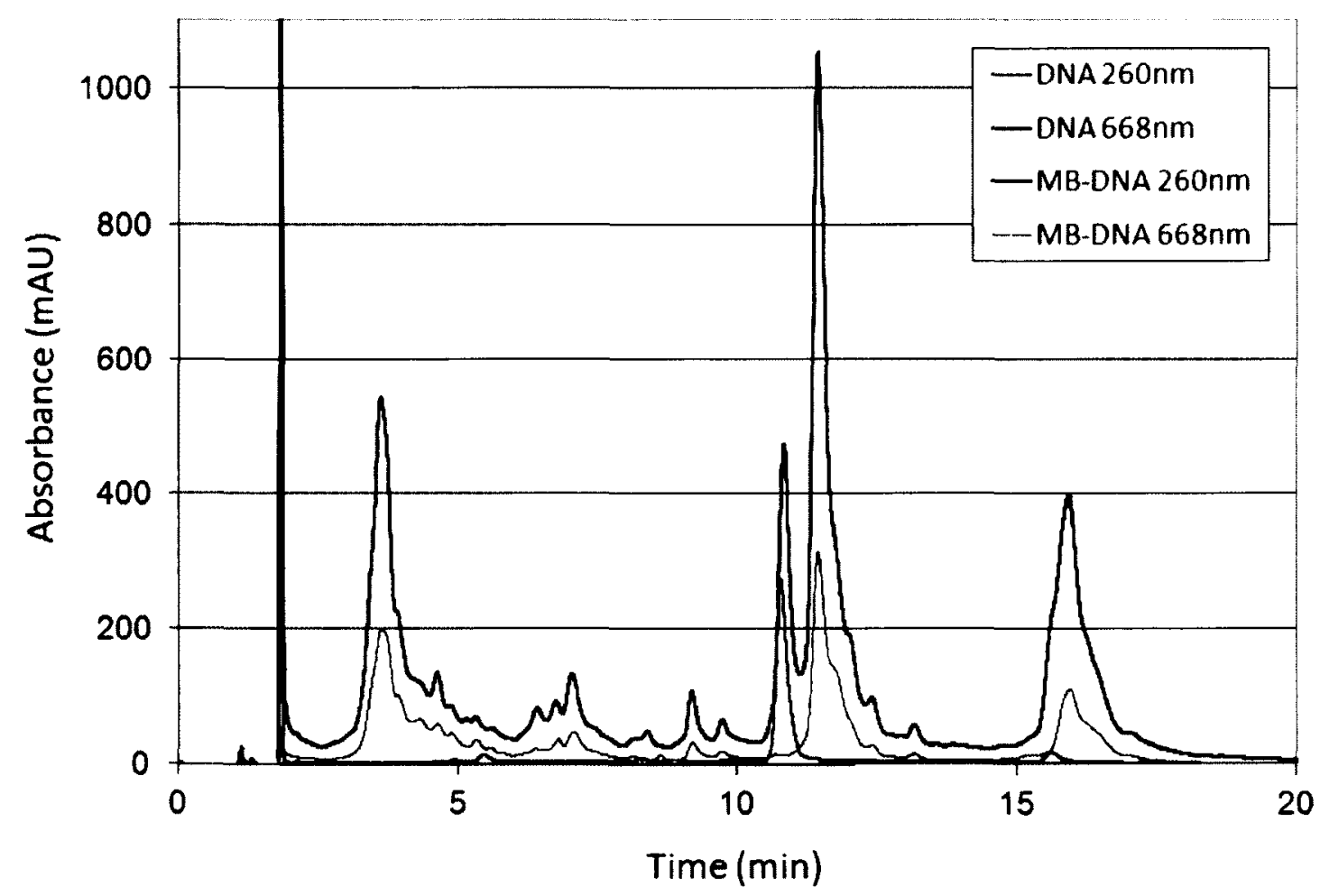

Figure 2-16: RP-HPLC chromatograms of unlabeled thrombin aptamer (DNA) and methylene blue conjugation products (MB-DNA). UV-signals at $260 \mathrm{~nm}$ and $668 \mathrm{~nm}$ plotted for each.

As expected, short retention time failure sequences were also modified with methylene blue due to the use of 3'-amino modified CPG supports. Full-length unlabeled thrombin aptamer was found to have a retention time of 11 minutes based on the overlap of DNA and MB-DNA signals in Figure 2-16. The desired methylene blue-labeled full length product had a retention time of 11.5 minutes. Collections at these retention times were analyzed by UV-Vis. ESI-MS analysis supported the peak identification. 


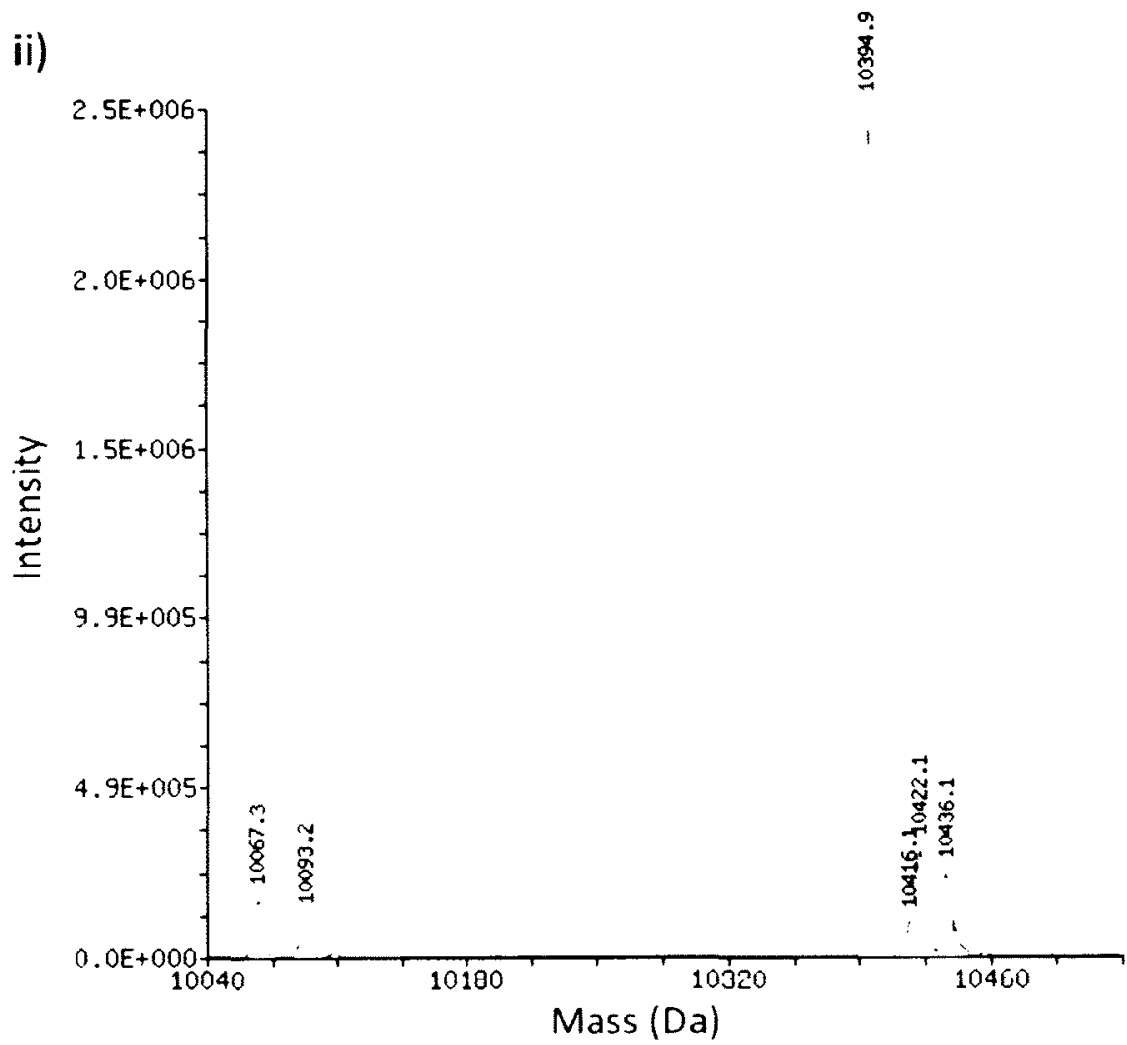

Figure 2-17: RP-HPLC Collection at 11 minutes: i) UV-Visible spectrum, ii) Deconvoluted ESI mass spectrum 
i)

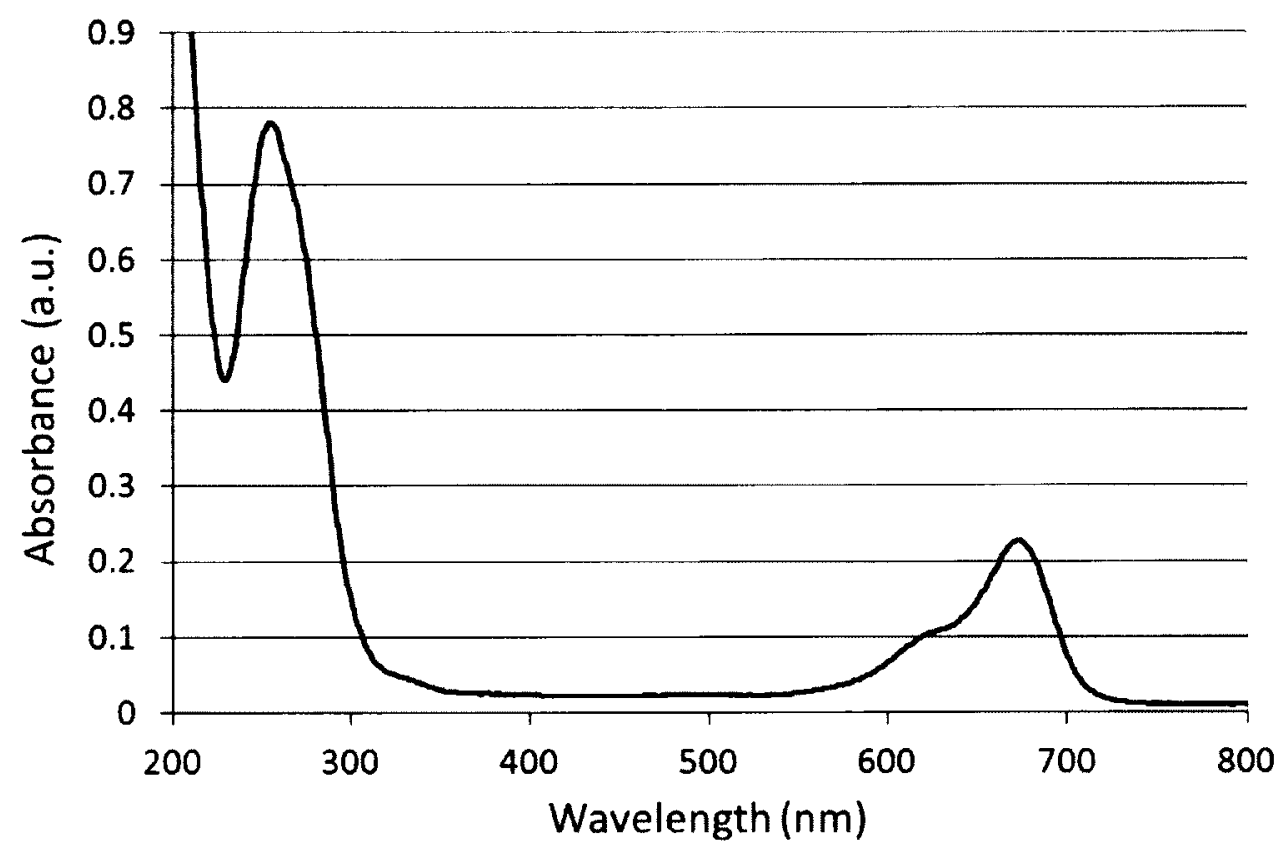

ii)

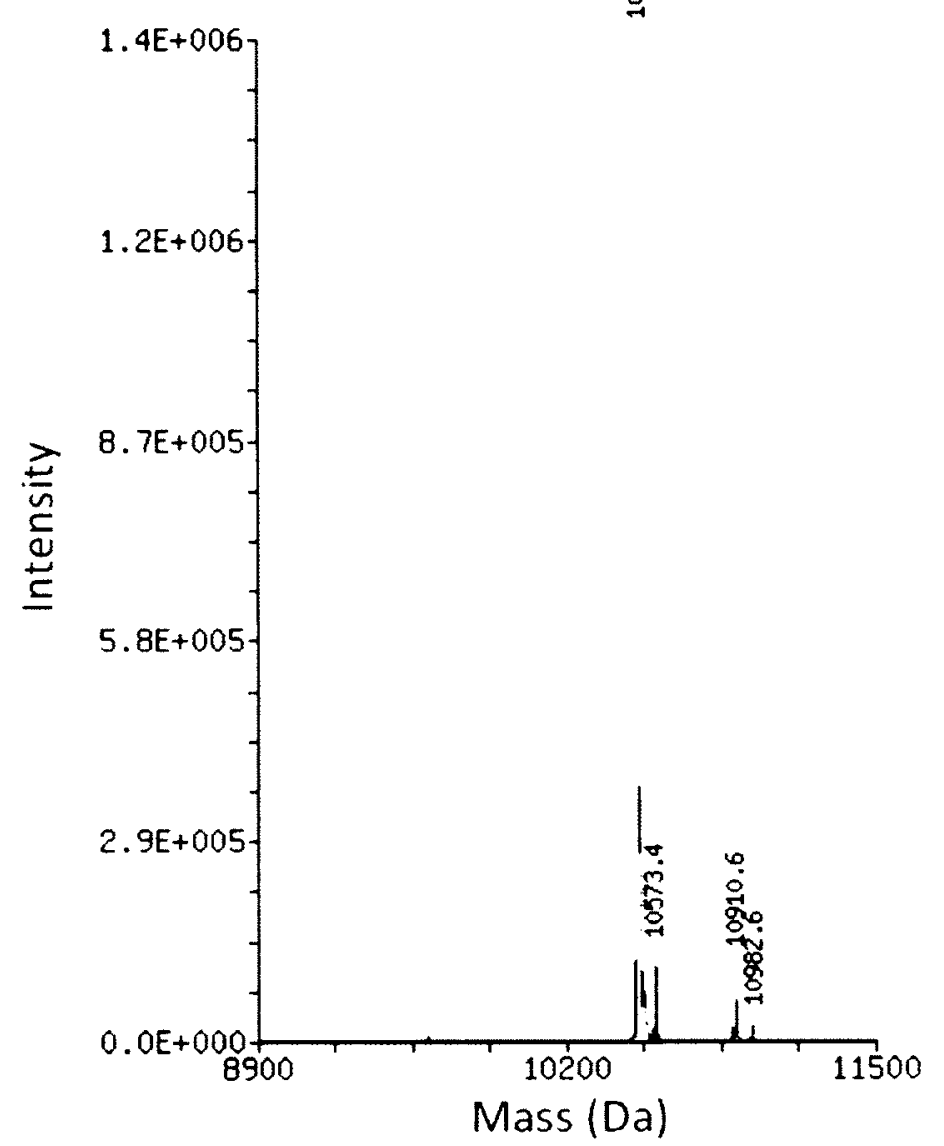

Figure 2-18: RP-HPLC Collection at 11.5 minutes: i) UV-Visible spectrum, ii) Deconvoluted ESI mass spectrum 
Figures 2-17 and 2-18 contain the UV-Visible and ESI mass spectra of RP-HPLC collections at 11 minutes and 11.5 minutes respectively. The somewhat asymmetric appearance of the DNA absorption at 260nm in Figure 2-17 (i) was to be expected given the G-rich thrombin aptamer sequence ${ }^{100}$. The expected mass of the unlabeled thrombin aptamer without protecting groups: HO- $\left(\mathrm{CH}_{2}\right)_{6}$-S-S- $\left(\mathrm{CH}_{2}\right)_{6}-5$ '-TAA GTT CAT CTC CCC GGT TGG TGT GGT TGG T- $\mathrm{CH}_{2}-\mathrm{CH}\left(\mathrm{CH}_{2} \mathrm{OH}\right)-\left(\mathrm{CH}_{2}\right)_{4}-\mathrm{NH}_{2}-3$ ', was 10092Da. Retention of the 5'-DMT raises this mass to $10394.5 \mathrm{Da}$. Peaks corresponding to both of these masses were observed in the mass spectrum of the fraction collected at 11 minutes. Strict quantitative information should not be inferred from these ESI-MS experiments due to potential differences in efficiency of ionization and transmission of the different species $^{101}$. However, the peak at $10394.5 \mathrm{Da}$ was of much larger magnitude, suggestive of only a minor amount of spontaneous detritylation between fraction collection and analysis.

The UV-Vis spectra of the fraction collected at 11.5 minutes shows the expected absorbance ratio based on the extinction coefficients of DNA and MB to support their presence in a $1: 1$ conjugate $\left(\varepsilon_{260 \mathrm{~nm}}=308883 \mathrm{M}^{-1} \mathrm{~cm}^{-1} / \varepsilon_{668 \mathrm{~nm}}=81600 \mathrm{M}^{-1} \mathrm{~cm}^{-1} \approx 3.8\right)^{102,103}$. The expected mass of the MB-DNA conjugate, 10502Da, was readily identified as the major peak in the mass spectrum of this collection. 


\subsubsection{Ellman's reaction}

With the desired MB-DNA conjugate isolated, Ellman's reaction was used to determine if the functionality of the thiol modification had been preserved ${ }^{104}$. This test was based on the reaction of thiolated DNA with the DTNB probe reagent to form equivalents of TNB anion which could be measured by UV-Vis at $412 \mathrm{~nm}^{104}$. First, TCEP was used to expose the thiol modification by cleaving the disulfide-bound protecting group. Upon the addition of one equivalent of DTNB, approximately two equivalents of $\mathrm{TNB}^{2-}$ were produced, suggesting that residual TCEP could simply be cleaving the DTNB reagent. Additional DTNB was added to raise the molar ratio of DTNB:DNA to 5:1, then $9: 1 . \mathrm{TNB}^{2-}$ formation peaked at approximately three equivalents. However, from this data alone it could not be concluded whether the thiol modification was responsible for any of the $\mathrm{TNB}^{2-}$ produced.

Two routes were considered from this point. The first would be to attempt isolation of a TNB-DNA adduct by RP-HPLC, monitoring for attached TNB at $325 \mathrm{~nm}^{105}$. The second was to analyze the reaction products directly by ESI-MS. The latter route was chosen, with the mass spectrum presented as Figure 2-19 (ii). 

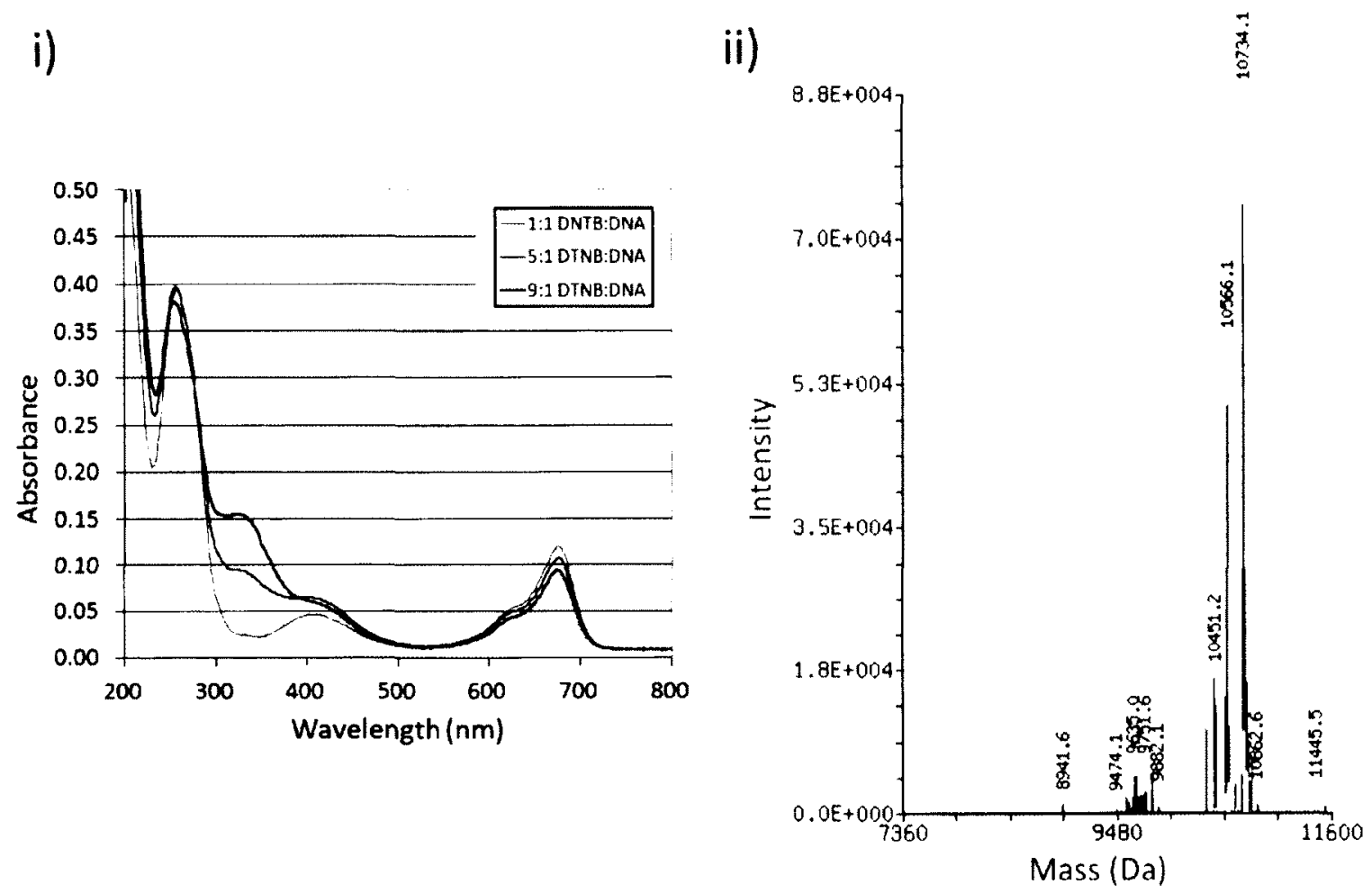

Figure 2-19: Results of the Ellman reaction: i) UV-Visible spectra of the products using 1:1, 5:1, and 9:1 molar ratios of DTNB reagent to DNA, ii) Deconvoluted ESI mass spectrum of 9:1 DTNB:DNA reaction products.

The expected mass of the TNB adduct was 10566Da. This was indeed one of the major peaks in the mass spectrum. The other major peak at 10734.1Da would correspond to an adduct with hexafluoroisopropanol (HFIPA, mass 168Da), an acid in the volatile buffer systems used in sample preparation by Novatia ${ }^{101}$. With this result in hand, the MB-DNA was deemed suitable for deposition onto gold electrodes. 


\subsubsection{Other approaches to MB-DNA synthesis and purification}

Efforts were made to purify the DNA prior to the conjugation reaction to prevent consumption of DCMB-SE by failure sequences and to simplify downstream cleanup. While the methods used excelled at removing failure sequences, there were several reasons why it was found best to proceed directly to the conjugation reaction with crude DNA synthesis products. Most importantly, the reliance on tris buffer and the subsequent inability to remove the primary amine was most detrimental to the success of the conjugation reaction. Also, during PAGE, it was possible that the amino modification could be inactivated by reaction with unpolymerized acrylamide ${ }^{106}$.

Additional methods were also attempted for purification after the conjugation reaction. PAGE was deemed to have insufficient resolution for separating unlabeled DNA sequences from the desired product, at least at the $19 \%$ acrylamide concentration used. Bio Spin 6 columns, based on the principles size exclusion chromotagraphy to separate smaller components, were also inadequate for purification. This was not surprising, as the hydrodynamic volume of methylene blue-labeled thrombin aptamer

should not be very different from that of the unlabeled thrombin aptamer ${ }^{107}$. Amicon desalting columns were also found to be insufficient. Product loss during repeated washings, with both deionized water and $140 \mathrm{mM} \mathrm{NaCl}$, negated the minimal improvements in the ratio of methylene blue to DNA measured in the retentate. Table 2-7 summarizes the alternate purification methods used. 
Table 2-7: Summary of alternate methods attempted in the synthesis and purification of MB-DNA.

\begin{tabular}{|l|l|l|}
\hline Purification method & $\begin{array}{c}\text { Suspected difficulties if used } \\
\text { before conjugation }\end{array}$ & $\begin{array}{l}\text { Suspected difficulties if } \\
\text { used after conjugation }\end{array}$ \\
\hline Clarity QSP cartridges & $\begin{array}{l}\text { Tris quenching of conjugation } \\
\text { reaction. Used tris buffer after } \\
\text { deprotection reaction to ensure } \\
\text { retention of 5'-trityl. Tris present } \\
\text { in loading, wash, and elution } \\
\text { buffers. }\end{array}$ & N/A \\
\hline $\begin{array}{l}\text { Poly(acrylamide) gel } \\
\text { electrophoresis }\end{array}$ & $\begin{array}{l}\text { Inactivation of amino } \\
\text { modification by reaction with } \\
\text { unpolymerized acrylamide. Tris } \\
\text { present in polymerization and } \\
\text { running buffers. }\end{array}$ & $\begin{array}{l}\text { Resolution insufficient for } \\
\text { isolation of desired product } \\
\text { ased. acriamide percentage } \\
\text { used. }\end{array}$ \\
\hline Bio Spin 6 columns & N/A & $\begin{array}{l}\text { Size exclusion separation } \\
\text { principle insufficient for } \\
\text { removal of electrostatically } \\
\text { bound methylene blue. }\end{array}$ \\
\hline $\begin{array}{l}\text { Amicon Ultra desalting } \\
\text { columns }\end{array}$ & N/A & $\begin{array}{l}\text { Excessive product loss with } \\
\text { repeated washings coupled } \\
\text { with only minimal removal } \\
\text { of excess methylene blue. }\end{array}$ \\
\hline
\end{tabular}

The conjugation reaction was also attempted using HEPES buffer, and DMSO to solubilize DCMB-SE. It can only be presumed that strictly anhydrous conditions were not maintained during these reaction attempts. After the successful conjugation reactions with the sodium phosphate conjugation buffer and DMF system of Schubert et al., these alternate choices were not re-explored ${ }^{98}$. Lower molar excesses of DCMB-SE to DNA also produced poor results. Since a 3'-amino modification was used, and with the absence 
of pre-purification, all failure sequences would be available for reaction. Based on steric arguments, it is plausible that the failure sequences would be more reactive than the fulllength thrombin aptamer toward DCMB-SE. Adequate labeling of the full-length sequence was observed at 4:1 DCMB-SE:DNA.

\subsubsection{Preparation and electrochemical characterization of modified electrodes}

Gold electrodes were mechanically polished with alumina particles, sonicated in ethanol, and electrochemically cleaned by cyclic voltammetry in sulfuric acid. Once cleaned, electrodes were immersed in a solution of reduced MB-DNA or SH-DNA for 48 hours. Then, electrodes were immersed in an ethanolic solution of 6-mercapto-1-hexanol $(\mathrm{MCH})$ to block any vacant portions of the gold surface. Figure 2-20 presents the cyclic voltammogram of a MB-DNA modified electrode recorded immediately after the DNA deposition step, and again after formation of the backfilling layer.

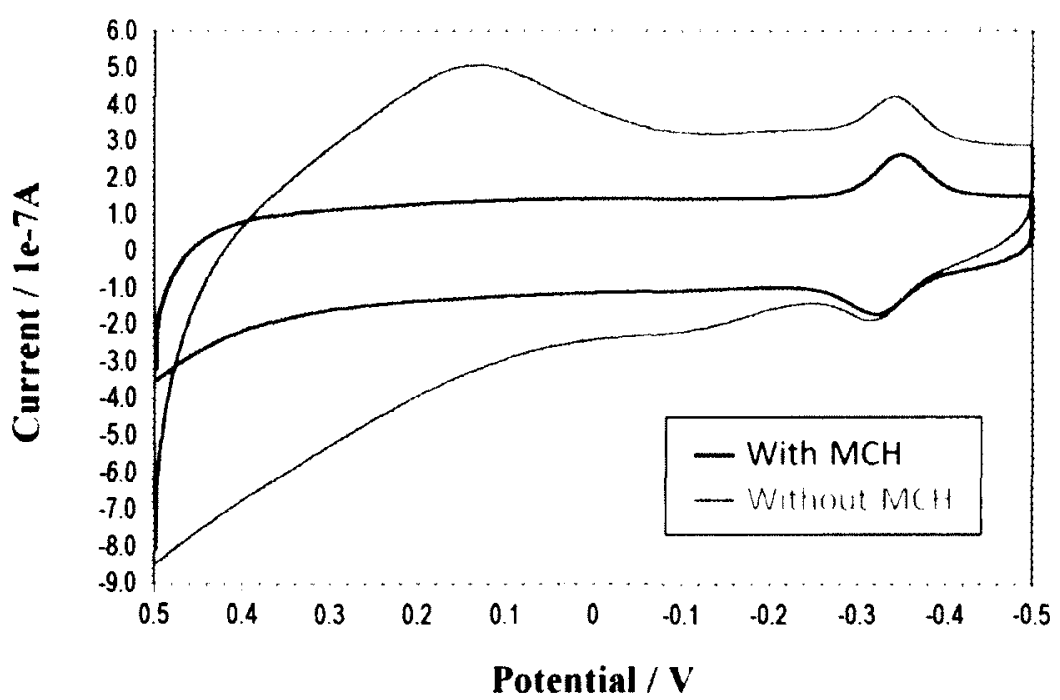

Figure 2-20: Cyclic voltammograms of MB-DNA modified electrodes in $10 \mathrm{mM}$ tris with (blue) and without (red) a backfilling layer of 6-mercapto-1-hexanol (MCH). 
The effectiveness of the backfilling layer to block a large portion of the background charging current was comparable to literature expectations ${ }^{65}$. The $\mathrm{MCH}$ deposition step also has the additional advantage of displacing any non-specifically bound DNA, leaving only surface-bound DNA that is available for interaction with target $^{108}$. Deposition of thiolated compounds on gold surfaces has been reported to occur in two stages, a quick initial adsorption where up to $90 \%$ of the layer is formed, followed by a slow reorganization step lasting many hours ${ }^{109,110}$. To form the best monolayers possible, DNA and $\mathrm{MCH}$ depositions were therefore performed with deposition times of 48 hours and 24 hours respectively. A high ionic strength deposition buffer was used to mediate electrostatic repulsion between DNA strands, promoting formation of more dense monolayers ${ }^{110}$.

Next, the surface coverage of the formed monolayers was determined. In the case of methylene blue covalently bound to DNA in a 1:1 ratio, the coverage could be calculated directly from the equation:

$$
\Gamma_{D N A}=\frac{Q_{c}}{n F A}
$$

where $\mathrm{Q}_{\mathrm{c}}$ is the integrated area of the cathodic peak, $\mathrm{n}$ the number of electrons in the reaction, $\mathrm{F}$ the Faraday constant, and $\mathrm{A}$ the surface area of the gold electrode ${ }^{95}$. MB-DNA surface coverages were on the order of $10^{-12} \mathrm{~mol} \cdot \mathrm{cm}^{-2}$, comparable to literature values ${ }^{95}$. For example, a coverage of $3.5 \times 10^{-12} \mathrm{~mol} \cdot \mathrm{cm}^{-2}$ was calculated for the surface characterized in Figure 2-20, and coverages between 3.0- and $8.0 \times 10^{-12} \mathrm{~mol} \cdot \mathrm{cm}^{-2}$ were obtained throughout the course of the present work. 
Since the depositions were performed with the same concentrations, buffers, and reaction times, it could be presumed that SH-DNA surface coverage would be very similar to that of MB-DNA. Nevertheless, to obtain a more precise estimate, electrostatic interaction between surface bound SH-DNA a ruthenium hexammine probe was employed to quantify surface coverage. For non-covalent probes occupying multiple charges on the phosphate backbone, the surface coverage may be calculated by manipulation of the above equation to the following:

$$
\Gamma_{D N A}=\Gamma_{\text {Probe }} \frac{z}{m}=\frac{Q_{c}}{n F A} \frac{z}{m}
$$

where $\mathrm{z}$ is the charge of the redox-active probe and $\mathrm{m}$ the number of phosphates available in the DNA strand ${ }^{111}$. Indeed, via the ruthenium hexammine test, surface coverage for SH-DNA monolayers was found to be on the same order as MB-DNA monolayers.

As an additional qualitative measure of SH-DNA surface coverage, cyclic voltammetry was also used to compare the response to $\mathrm{K}_{3} \mathrm{Fe}(\mathrm{CN})_{6}$ of an SH-DNA modified surface to that of a bare gold electrode. A high SH-DNA surface coverage would inhibit signal from the anionic redox couple due to electrostatic repulsion from the phosphate backbone ${ }^{84}$. Figure 2-21 demonstrates that the surface coverages obtained here were sufficiently high for this purpose, that a monolayer inaccessible to $\mathrm{Fe}(\mathrm{CN})_{6}{ }^{3 \cdot}$ $/ \mathrm{Fe}(\mathrm{CN})_{6}{ }^{4 \cdot}$ had been formed. 

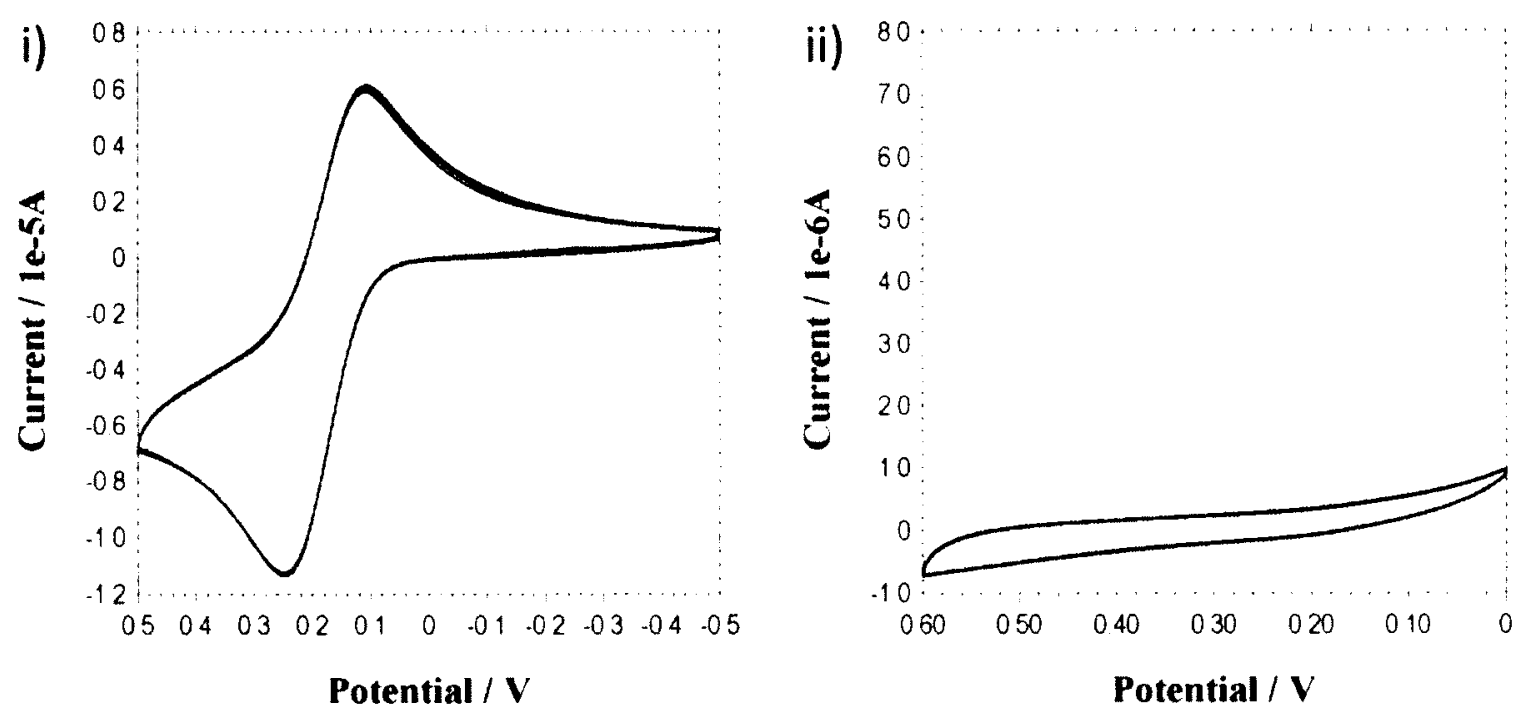

Figure 2-21: Qualitative analysis of SH-DNA surface coverage by cyclic voltammetry. Response to $2 \mathrm{mM} \mathrm{K}_{3} \mathrm{Fe}(\mathrm{CN})_{6}$ of a (i) bare gold electrode, (ii) SH-DNA modified gold electrode.

This result would also be important in the subsequent sections where the reduction of $\mathrm{Fe}(\mathrm{CN})_{6}{ }^{3-}$ is catalyzed by the methylene blue of MB-DNA. Since a DNA monolayer backfilled with $\mathrm{MCH}$ appears to prevent signaling from $\mathrm{Fe}(\mathrm{CN})_{6}{ }^{3-} / \mathrm{Fe}(\mathrm{CN})_{6}{ }^{4-}$, changes in electrode response could be more directly attributed to reactions involving methylene blue.

\subsubsection{Optimization of thrombin incubation time and the role of cations}

The time dependence of sensor response to thrombin was investigated in order to select an optimal incubation time for future measurements. This was done by conducting a square wave voltammetry sweep every five minutes in the presence of $350 \mathrm{nM}$ thrombin. Figure 2-22 presents the results, with only voltammograms at major time intervals shown for clarity. 


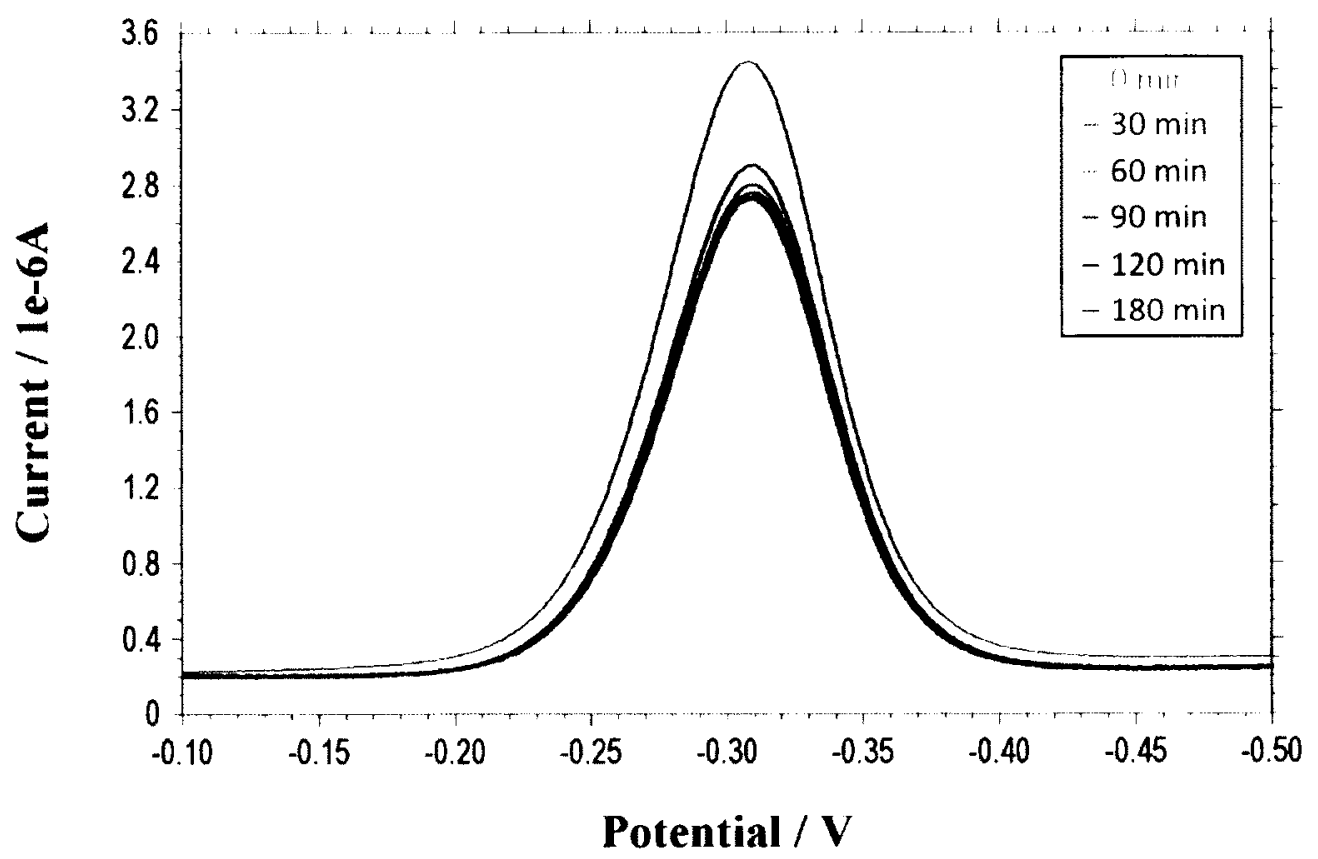

Figure 2-22: Time course study by square wave voltammetry of a MB-DNA modified electrode in the presence of $350 \mathrm{nM}$ thrombin protein.

This experiment was repeated in the absence of thrombin with a freshly prepared electrode featuring a similar surface coverage. This was to identify any contribution to the observed signal-off trend by accumulation of charge at the surface resulting from conducting the scans multiple times, increasing resistance to the redox activity of methylene blue ${ }^{65}$. The results of these studies in both TBB and NoKTBB buffers are plotted in Figure 2-23. 


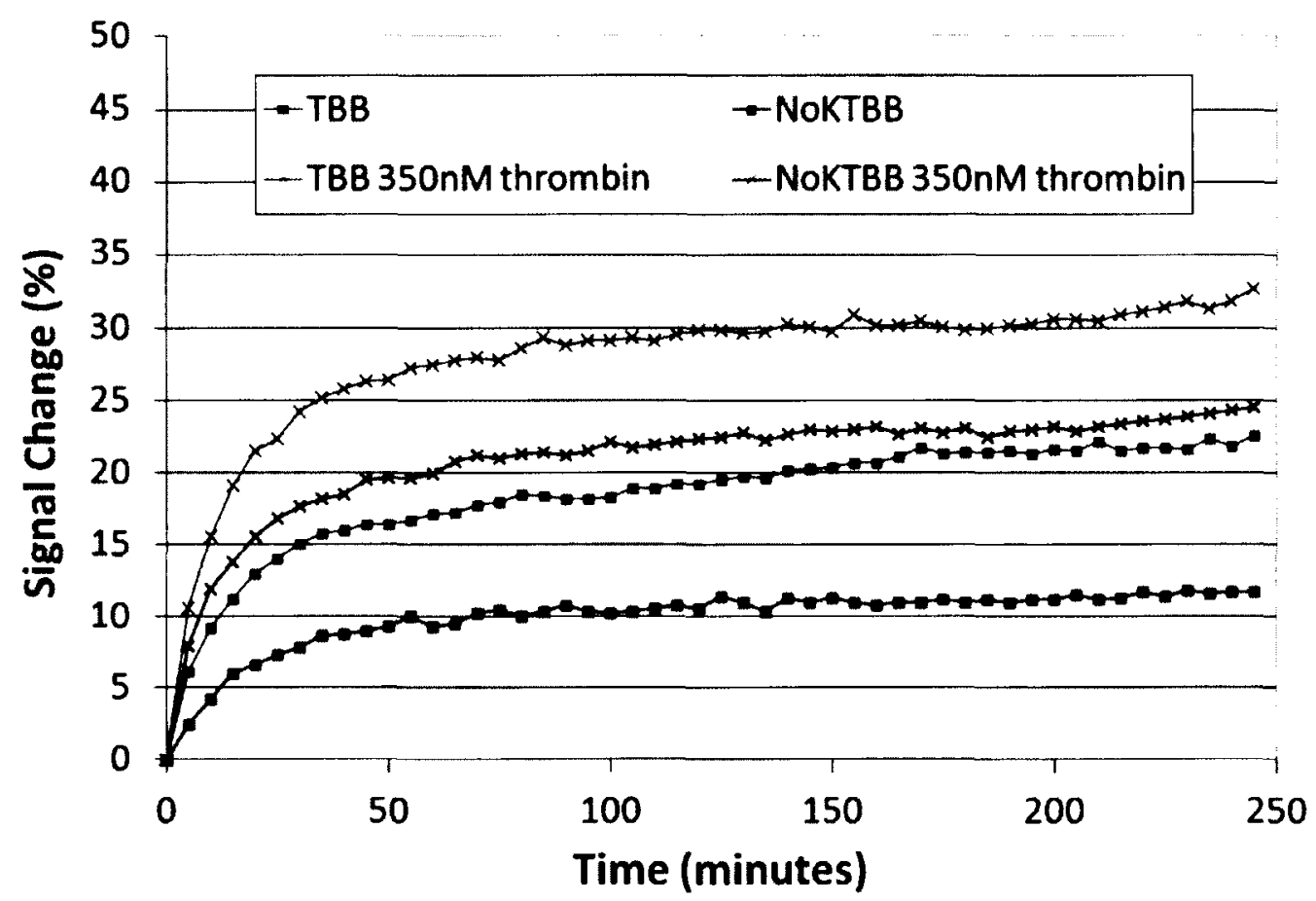

Figure 2-23: Time course study by square wave voltammetry in the presence and absence of 350nM thrombin target for both thrombin binding buffer (TBB) and its mimic (NoKTBB).

Based on the results presented in Figures 2-22 and 2-23, a thrombin incubation time of one hour was deemed suitable for sensor operation. Minimal changes in signal after this time were presumably due to the aforementioned charging currents from repeatedly running the SWV experiment, not further progression toward a thrombinaptamer binding equilibrium. Figure 2-23 also reinforced the importance of considering cations such as potassium and magnesium when describing the behavior of the thrombin binding aptamer ${ }^{112.113}$. Considering a cross section at one hour, the relative signal change between the absence and presence of $350 \mathrm{nM}$ thrombin target was approximately $10 \%$ for both TBB and NoKTBB buffers. However, the absolute changes were significantly higher for TBB. This is proposed to be a result of potassium and magnesium ions stabilizing G-quadruplex structures among free thrombin aptamers, inducing further 
signal change in comparison to the NoKTBB experiment. Support for this hypothesis arises from the instances where thrombin binding aptamers have been put forward as potassium sensors, such as the work of Radi et $\mathrm{al}^{113}$. The results of these time course studies would be important moving forward, as they indicated that high concentrations of potassium and magnesium were not required to induce thrombin-aptamer binding.

\subsubsection{Determination of conditions for electrocatalysis}

Having determined the optimal incubation time for thrombin detection experiments, the next objective was to determine appropriate conditions for the catalytic reduction of $\mathrm{Fe}(\mathrm{CN})_{6}{ }^{3-}$ by methylene blue. As a first step, the thrombin binding buffer used successfully by Xiao et al. for the original thrombin aptasensor was employed ${ }^{87}$. Cyclic voltammetry was performed in TBB for both SH-DNA and MB-DNA modified electrodes over four orders of magnitude of $\left[\mathrm{K}_{3} \mathrm{Fe}(\mathrm{CN})_{6}\right]$, from $0.01-10 \mathrm{mM}$.
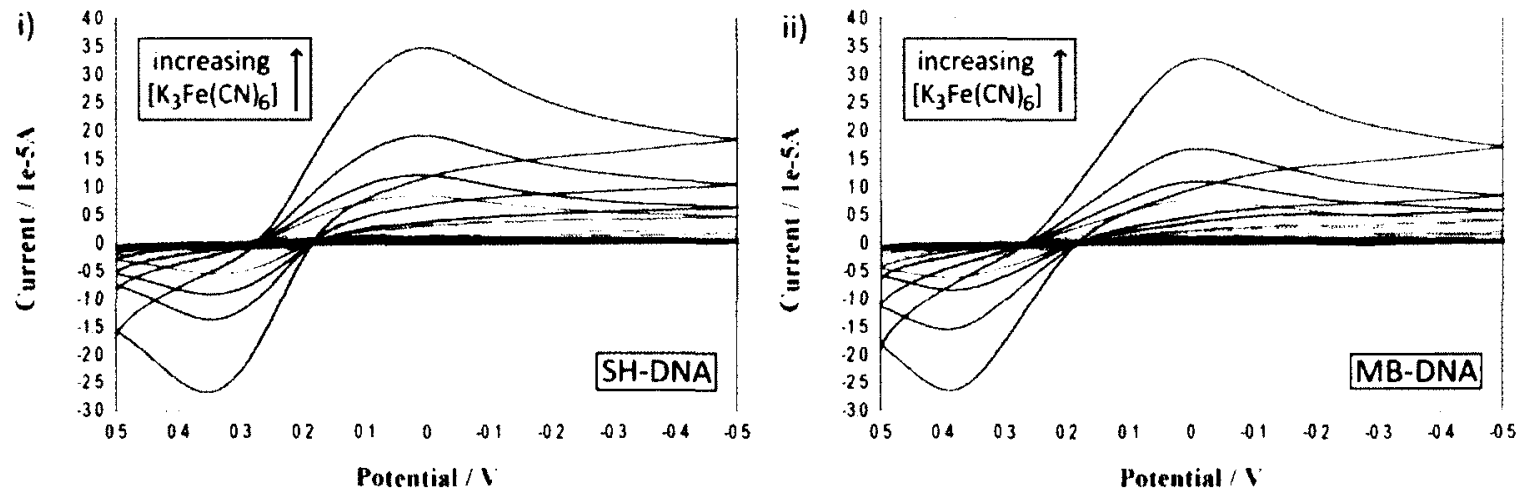

Figure 2-24: Cyclic voltammograms for (i) SH-DNA and (ii) MB-DNA modified electrodes with increasing concentrations of $\mathrm{K}_{3} \mathrm{Fe}(\mathrm{CN})_{6}$ in thrombin binding buffer (TBB). 
Figure 2-24 demonstrates that high concentrations of $\mathrm{K}_{3} \mathrm{Fe}(\mathrm{CN})_{6}$ resulted in very similar cyclic voltammograms for SH-DNA and MB-DNA modified electrodes. Figure 225 contains the cyclic voltammograms presented independently for the lower concentrations of $\mathrm{K}_{3} \mathrm{Fe}(\mathrm{CN})_{6}$. It was hypothesized that the high ionic strength of TBB passivated the negative charge of the DNA monolayer, allowing the anionic $\mathrm{Fe}(\mathrm{CN})_{6}{ }^{3-}$ $/ \mathrm{Fe}(\mathrm{CN})_{6}{ }^{4-}$ redox couple to be measured directly, with the resulting background current washing out any signal from methylene blue. The increased peak splitting in comparison to the bare gold electrode response from Figure 2-21 suggested that the DNA/MCH films impede the ability of the anions to reach the electrode surface to a certain extent ${ }^{11}$. 

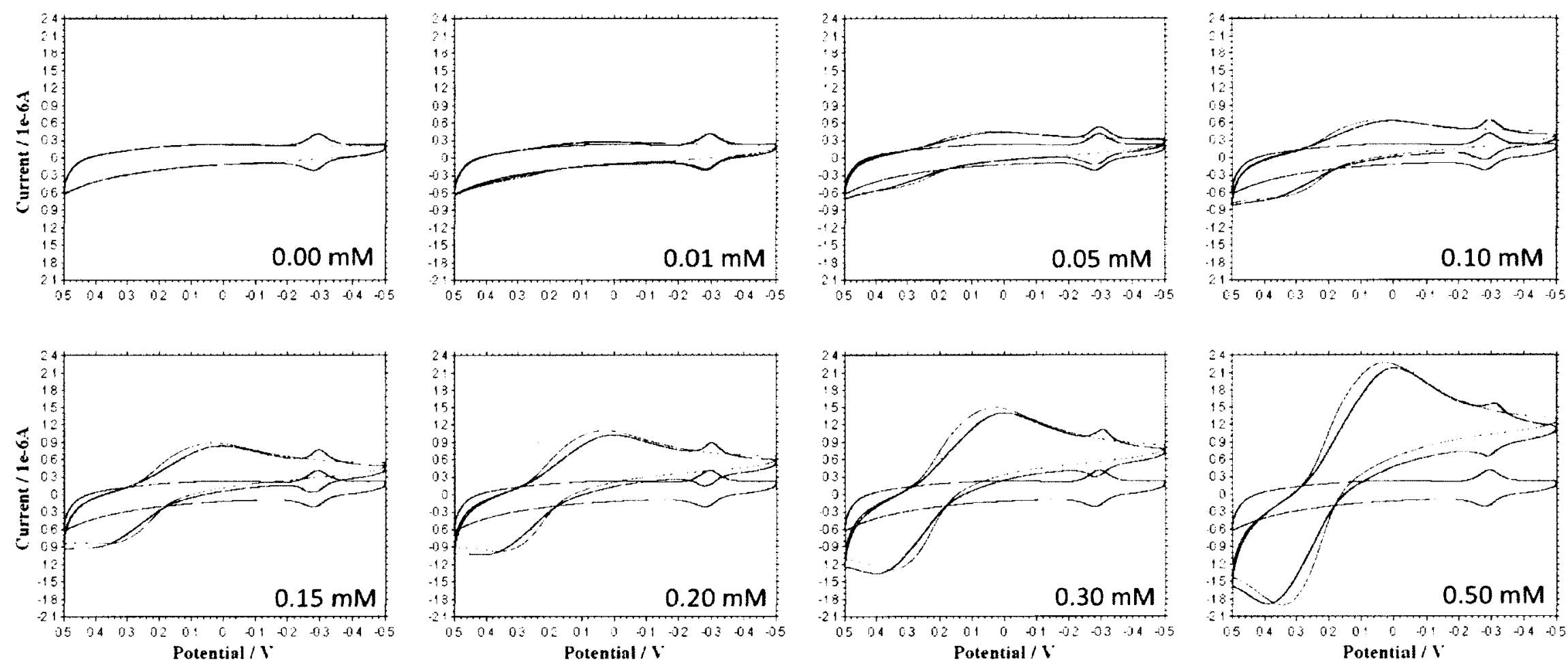

Figure 2-25: Cyclic voltammograms of SH-DNA (red) and MB-DNA (green) modified electrodes with $0.00-0.50 \mathrm{mM}$ $\mathrm{K}_{3} \mathrm{Fe}(\mathrm{CN})_{6}$ in TBB. The blue voltammogram corresponds to $\mathrm{MB}-\mathrm{DNA}$ in the absence of $\mathrm{K}_{3} \mathrm{Fe}(\mathrm{CN})_{6} . \mathrm{X}-$ and $\mathrm{Y}-\mathrm{scales}$ are identical in all 8 plots. 
In TBB, the integrated area of the methylene blue reduction peak was in fact found to decrease as increasing concentrations of $\mathrm{K}_{3} \mathrm{Fe}(\mathrm{CN})_{6}$ were added to the system, and at large concentrations no reduction peak was discernible. As this seemed detrimental to the goals of the project, new buffer systems were required.

Based largely on the successes of O'Sullivan and coworkers when using low ionic strength buffers without potassium or sodium for thrombin aptasensing, and the results described in Section 2.3.3, 10mM tris buffer was next explored ${ }^{91,112,113}$. The experiments were repeated exactly as performed for TBB using fresh electrodes with similar surface coverages. The results are presented in Figures 2-26 and 2-27.
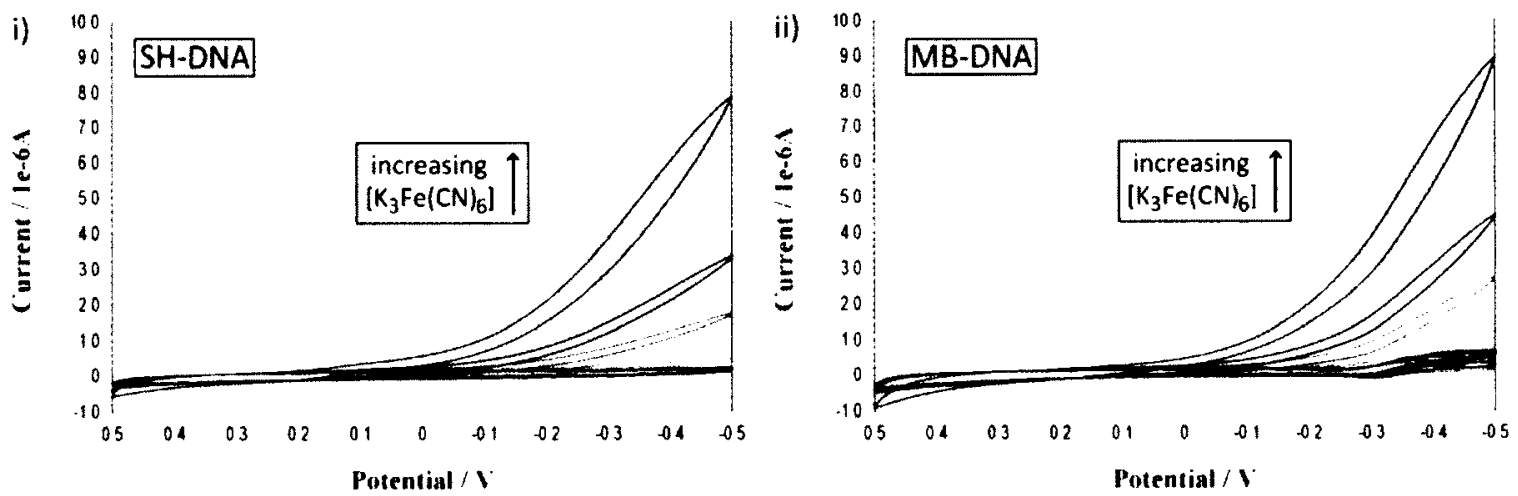

Figure 2-26: Cyclic voltammograms for (i) SH-DNA and (ii) MB-DNA modified electrodes with increasing concentrations of $\mathrm{K}_{3} \mathrm{Fe}(\mathrm{CN})_{6}$ in $10 \mathrm{mM}$ tris buffer. 

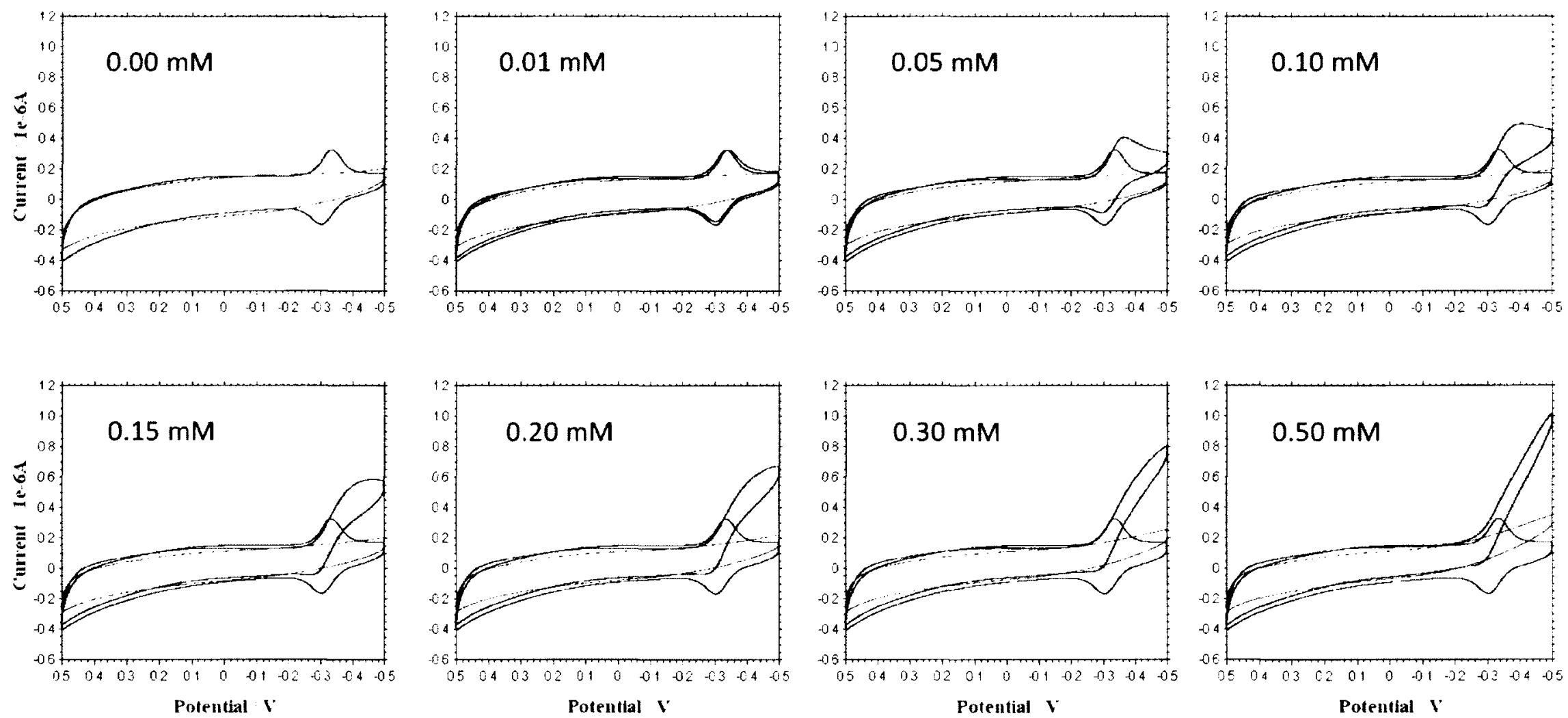

Figure 2-27: Cyclic voltammograms of SH-DNA (red) and MB-DNA (green) modified electrodes with $0.00-0.50 \mathrm{mM}$ $\mathrm{K}_{3} \mathrm{Fe}(\mathrm{CN})_{6}$ in $10 \mathrm{mM}$ tris buffer. The blue voltammogram corresponds to $\mathrm{MB}-\mathrm{DNA}$ in the absence of $\mathrm{K}_{3} \mathrm{Fe}(\mathrm{CN})_{6} . \mathrm{X}-$ and $\mathrm{Y}$ scales are identical in all 8 plots. 
As with TBB, the differences between SH-DNA and MB-DNA responses in tris buffer to $\mathrm{K}_{3} \mathrm{Fe}(\mathrm{CN})_{6}$ were much more obvious at the lower concentrations. Unlike the observations with TBB however, $E_{p, c}$ in tris buffer seemed to shift considerably upon the addition of $\mathrm{K}_{3} \mathrm{Fe}(\mathrm{CN})_{6}$, moving beyond the scan range above $0.20 \mathrm{mM}$. Opposite to the situation with TBB, it was considered that the low salt content of tris buffer maintained the repulsion between the negative DNA monolayer and solution borne $\mathrm{Fe}(\mathrm{CN})_{6}$ anions, bringing about the shift in $E_{p, c}{ }^{111}$. Another potential consideration in rationalizing these observations was the covalent nature of the attachment of methylene blue to the thrombin aptamer. In the electrocatalytic sensors of Kelley et al, methylene blue, once reduced to the neutral leucomethylene blue as shown in Figure 2-7, loses affinity for the DNA film and migrates into solution, where it can easily be oxidized back to methylene blue and return to the film ${ }^{85}$. Both the inability of leucomethylene blue to leave the monolayer, and the inability of $\mathrm{Fe}(\mathrm{CN})_{6}{ }^{3-}$ to approach the monolayer may have resulted in the extremely slow kinetics ${ }^{65}$.

To study the role of covalent versus non-covalent attachment, an experiment was conducted with a freshly prepared MB-DNA monolayer, but with 6uM free methylene blue and $2 \mathrm{mM} \mathrm{K}_{3} \mathrm{Fe}(\mathrm{CN})_{6}$ added to the system, mimicking the conditions of an electrocatalytic hybridization study by Fan and coworkers ${ }^{114}$. 


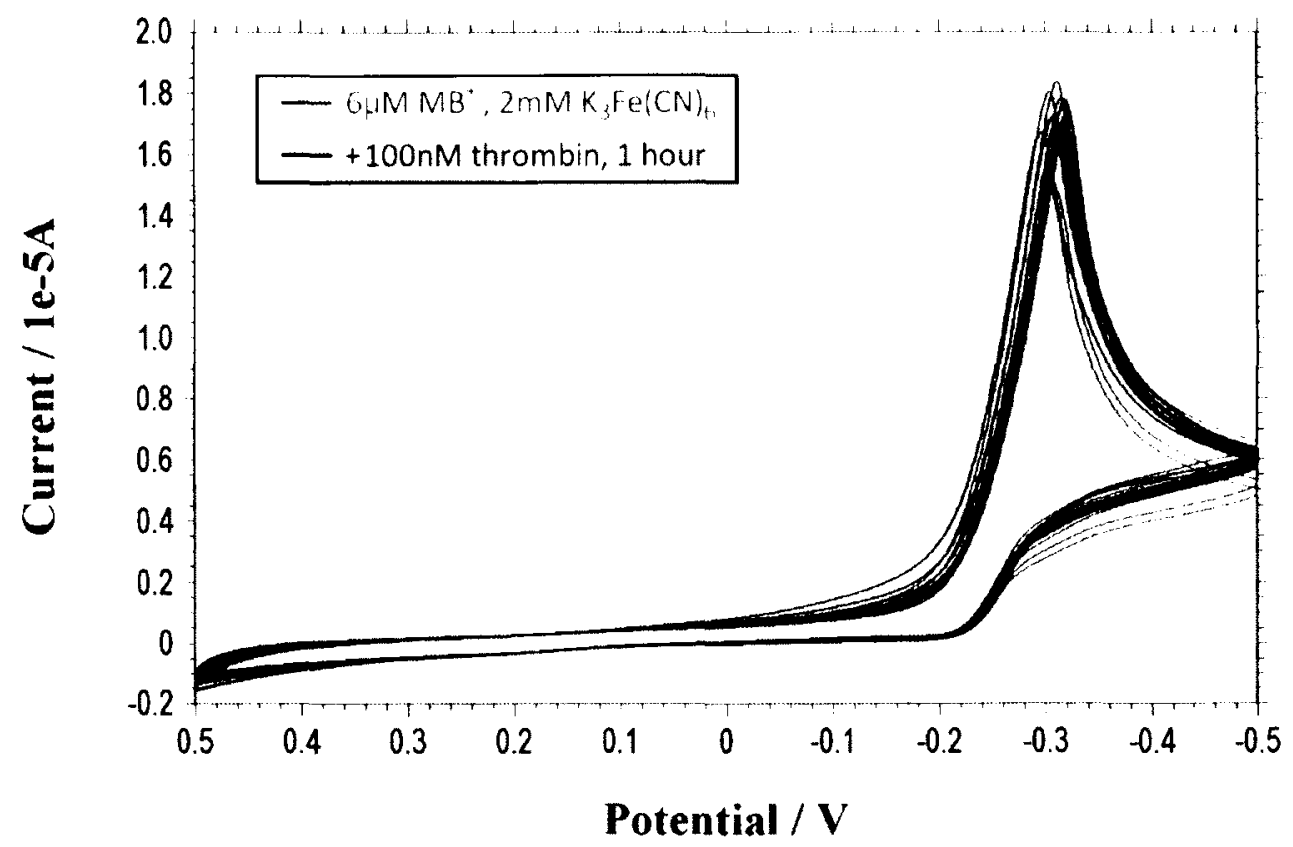

Figure 2-28: Electrocatalysis with non-covalent methylene blue $(6 \mu \mathrm{M})$ and $\mathrm{K}_{3} \mathrm{Fe}(\mathrm{CN})_{6}(2 \mathrm{mM})$ in $10 \mathrm{mM}$ tris buffer.

As can be seen in Figure 2-28, a large electrocatalytic enhancement of the methylene blue reduction peak was observed, eclipsing those produced with covalently bound methylene blue by an order of magnitude for equivalent $\mathrm{K}_{3} \mathrm{Fe}(\mathrm{CN})_{6}$ concentration $^{114}$. Unfortunately, the response to a fairly high thrombin concentration was minimal. Chronocoulometry experiments were also considered here, to exploit the considerable difference in peak current observed, but due to the scan to scan variation, selection of the appropriate voltage would be difficult, and likely introduce considerable error.

An intermediate ionic strength buffer was then selected, in an effort to minimize previous issues with passivation of the DNA monolayer at high ionic strength, and the inability to conduct electrocatalysis at low ionic strength with covalently bound 
methylene blue. For this purpose the PBS buffer employed by Kelley et al., containing $50 \mathrm{mM} \mathrm{NaCl}$ and $5 \mathrm{mM}$ phosphate, was used for the same set of cyclic voltammetry experiments as conducted with TBB and tris buffer ${ }^{82}$. As with the other buffers, Figure 2.29 also highlighted the similarity between SH-DNA and MB-DNA cyclic voltammograms at higher concentrations of $\mathrm{K}_{3} \mathrm{Fe}(\mathrm{CN})_{6}$. Additionally, it should be noted that at concentrations above $0.30 \mathrm{mM}$ the shape of the signals did not meet the apparent threshold at which the electrochemical workstation software automatically defines and integrates current peaks. Scanning beyond $-0.5 \mathrm{~V}$ in the negative direction was considered, but literature reports of desorbing gold-thiol bound DNA at extreme potentials discouraged these studies ${ }^{115-117}$. Also discouraging was the increasing similarity between the SH-DNA and MB-DNA responses, and considerably poorer reproducibility of peak shape at high $\left[\mathrm{K}_{3} \mathrm{Fe}(\mathrm{CN})_{6}\right]$.
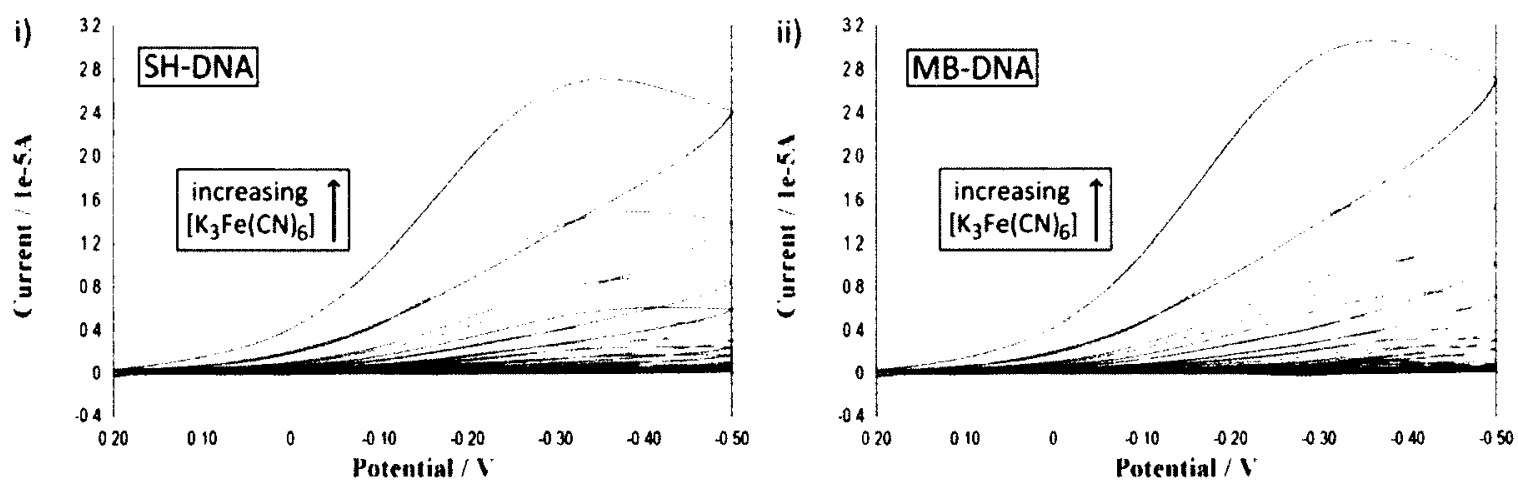

Figure 2-29: Cyclic voltammograms for (i) SH-DNA and (ii) MB-DNA modified electrodes with increasing concentrations of $\mathrm{K}_{3} \mathrm{Fe}(\mathrm{CN})_{6}$ in PBS. 
Values of $\left[\mathrm{K}_{3} \mathrm{Fe}(\mathrm{CN})_{6}\right]$ from $0.01-0.50 \mathrm{mM}$ were again more promising, as more significant differences could be identified between SH-DNA and MB-DNA modified electrode responses. These results are presented in Figure 2-30. The peak shapes were quite similar to literature expectations for electrocatalytic enhancement in PBS buffer, suggesting that covalent attachment was not a hindrance at this ionic strength, nor was a charge-neutralized DNA film simply bypassed by the $\mathrm{Fe}(\mathrm{CN})_{6}{ }^{4} / \mathrm{Fe}(\mathrm{CN})_{6}{ }^{3-}$ redox couple $^{82}$. From Figure 2-30, the concentration which seemed to display the best combination of an enhancement of cathodic peak area while maintaining a defined, fully integratable peak shape was $0.20 \mathrm{mM}$. This concentration would be used for thrombin sensing in PBS buffer. 

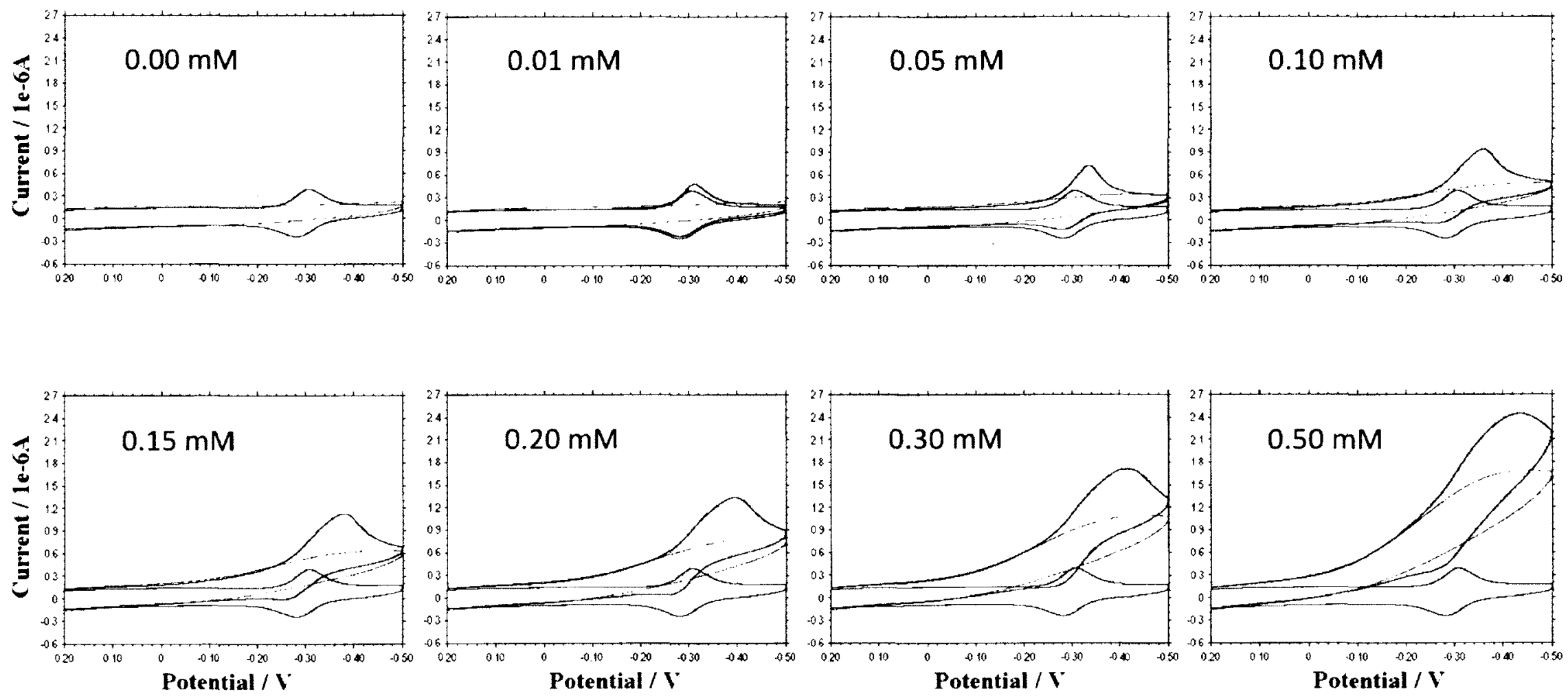

Figure 2-30: Cyclic voltammograms of SH-DNA (red) and MB-DNA (green) modified electrodes with $0.00-0.50 \mathrm{mM}$ $\mathrm{K}_{3} \mathrm{Fe}(\mathrm{CN})_{6}$ in PBS. The blue voltammogram corresponds to $\mathrm{MB}-\mathrm{DNA}$ in the absence of $\mathrm{K}_{3} \mathrm{Fe}(\mathrm{CN})_{6}$. $\mathrm{X}$ - and $\mathrm{Y}$ - scales are identical in all 8 plots. 


\subsubsection{Electrocatalytically enhanced aptasensor for thrombin}

Before demonstrating the utility of electrocatalysis to improve the state of electrochemical aptasensing, it was necessary to obtain a baseline level of performance by sensing thrombin directly with these MB-DNA modified electrodes. With the thrombin incubation time of one hour and appropriate PBS buffer selected, cyclic voltammetry was used for sensor operation. However, to maintain consistency with the intended electrocatalysis experiments, $0.6 \mathrm{mM}$ potassium chloride was added to compensate for the potassium to be introduced with $0.2 \mathrm{mM} \mathrm{K}{ }_{3} \mathrm{Fe}(\mathrm{CN})_{6}$. With a separate electrode possessing a similar surface coverage $\left(7.1\right.$ versus $\left.8.2 \mathrm{pmol} \cdot \mathrm{cm}^{-2}\right)$, the thrombin measurements were repeated in PBS buffer including $0.2 \mathrm{mM} \mathrm{K}_{3} \mathrm{Fe}(\mathrm{CN})_{6}$, with an incubation time of one hour. Thrombin measurements were reproduced using a separate electrode with $0.2 \mathrm{mM} \mathrm{K}_{3} \mathrm{Fe}(\mathrm{CN})_{6}$.
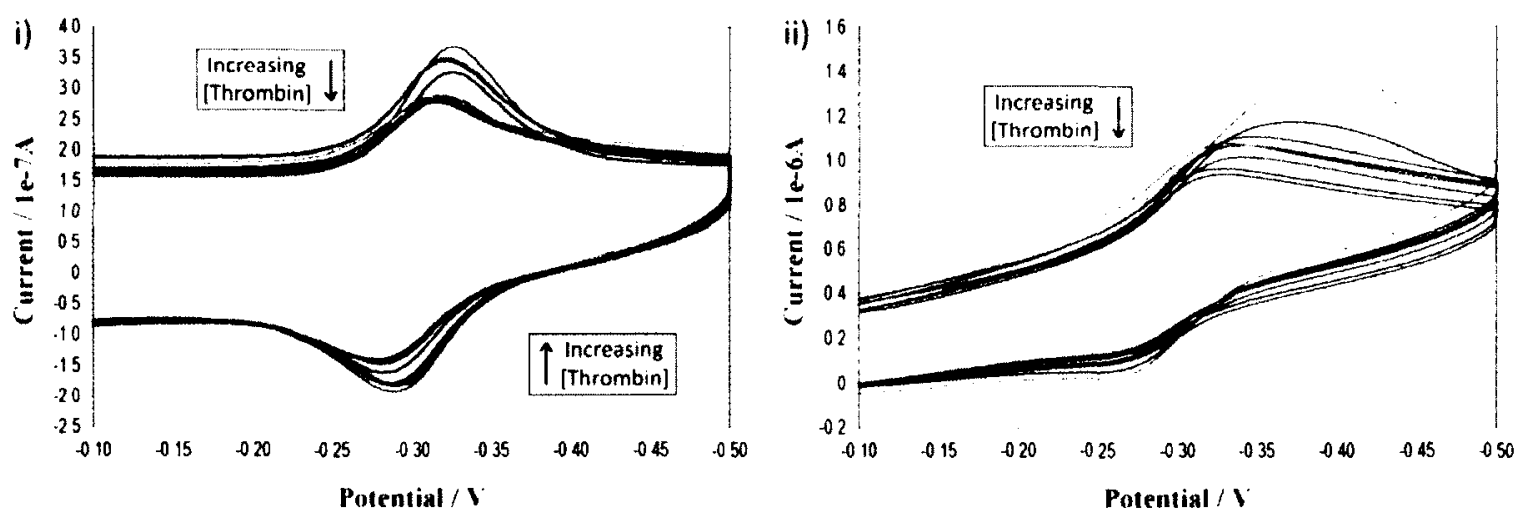

Figure 2-31: Cyclic voltammograms for increasing concentrations of thrombin in (i) PBS with $0.6 \mathrm{mM} \mathrm{KCl}$ and (ii) $\mathrm{PBS}$ with $0.2 \mathrm{mM} \mathrm{K}_{3} \mathrm{Fe}(\mathrm{CN})_{6}$. One hour incubation time preceded the acquisition for each concentration. 
The cathodic peaks were integrated using the electrochemical workstation software, and plotted against thrombin concentration in Figure 2-32. Slopes were calculated for the portions of the plots which appeared most linear for assessment of sensitivity.

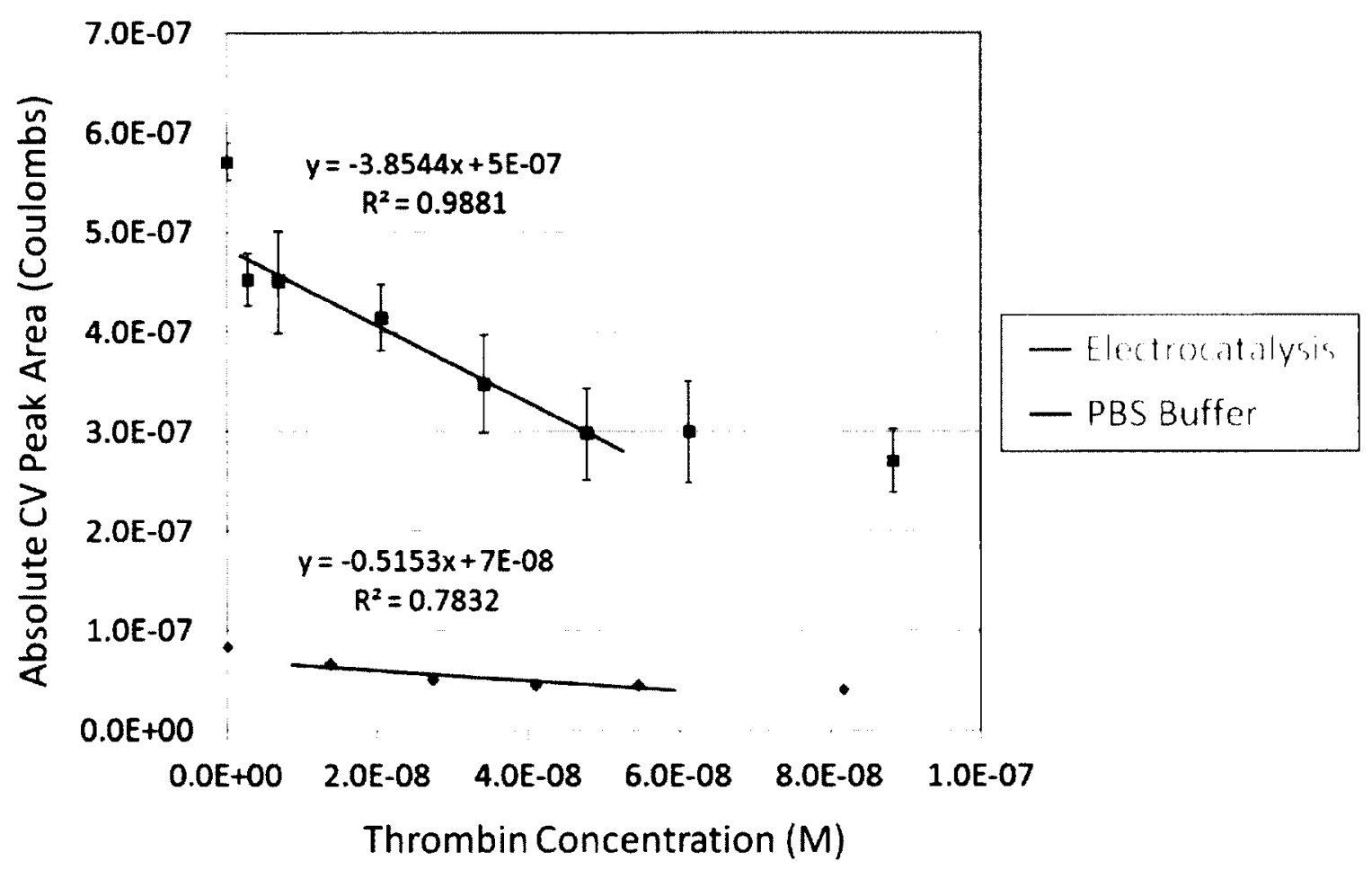

Figure 2-32: Integrated cathodic peaks from cyclic voltammetry experiments in the presence (red) and absence (blue) of $\mathrm{K}_{3} \mathrm{Fe}(\mathrm{CN})_{6}$.

Additionally, chronocoulometry was performed simultaneously with the replicated electrocatalysis study. After recording each cyclic voltammogram, $E_{p, c}$ was identified and used as the first potential step in a chronocoulometry experiment before continuing to the next thrombin concentration. The chronocoloumetry results are presented in Figure 2-33. 


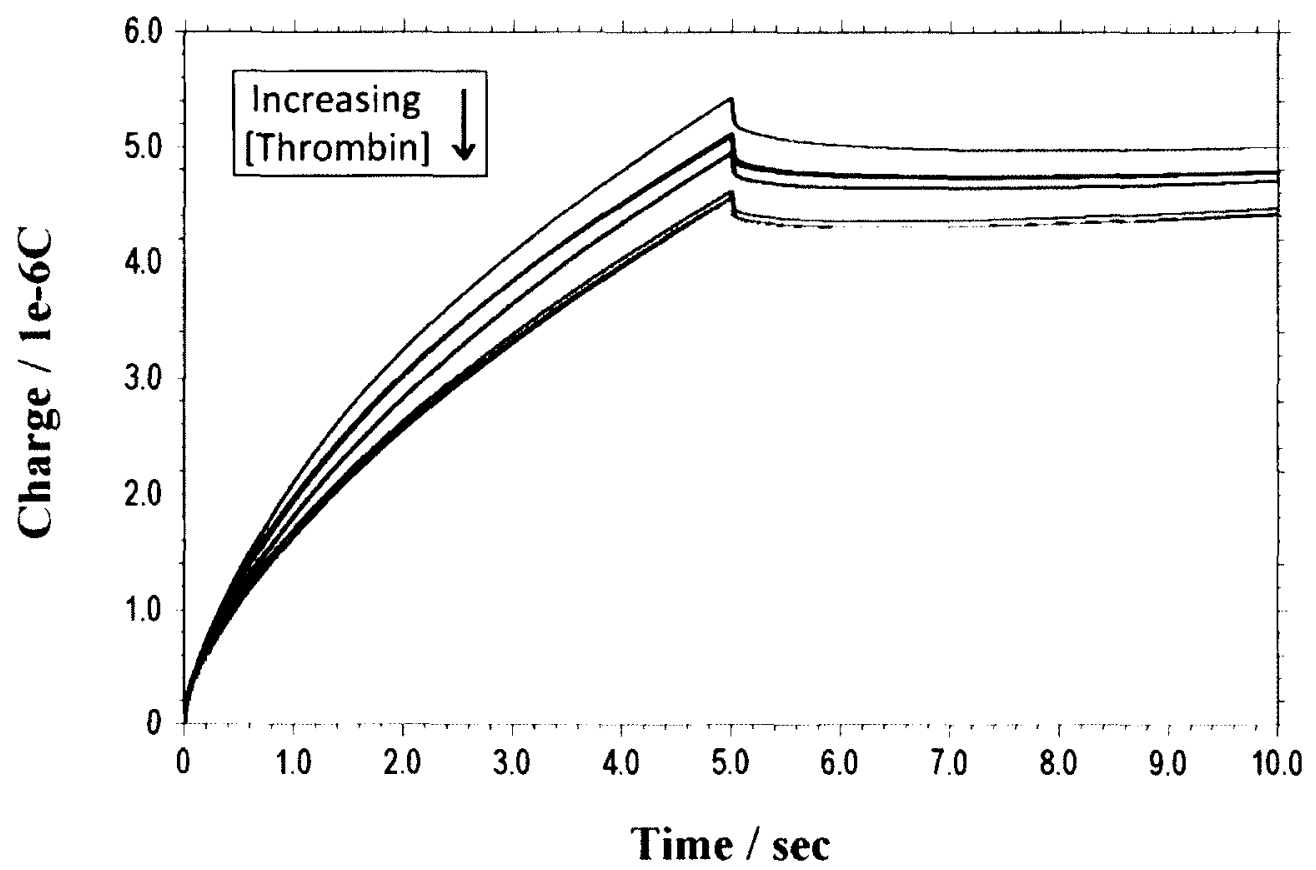

Figure 2-33: Chronocoulometry results for increasing concentrations of thrombin in PBS with $0.2 \mathrm{mM} \mathrm{K}_{3} \mathrm{Fe}(\mathrm{CN})_{6}$. Potential for the first step was set to match the cathodic peak maximum for the corresponding $\mathrm{CV}$ experiment.

The relative signal changes $\left(\Delta \mathrm{Q} / \mathrm{Q}_{0}\right)$ for both cyclic voltammetry and chronocoulometry experiments were calculated as:

$$
\frac{\Delta Q}{Q_{0}}=\frac{\left(Q_{0}-Q\right)}{Q_{0}}
$$

and plotted against thrombin concentration in Figure 2-34. Curves were generated by nonlinear least-squares fitting to a generic binding equation as described by Kemmer et $\mathrm{al}^{118}$. 


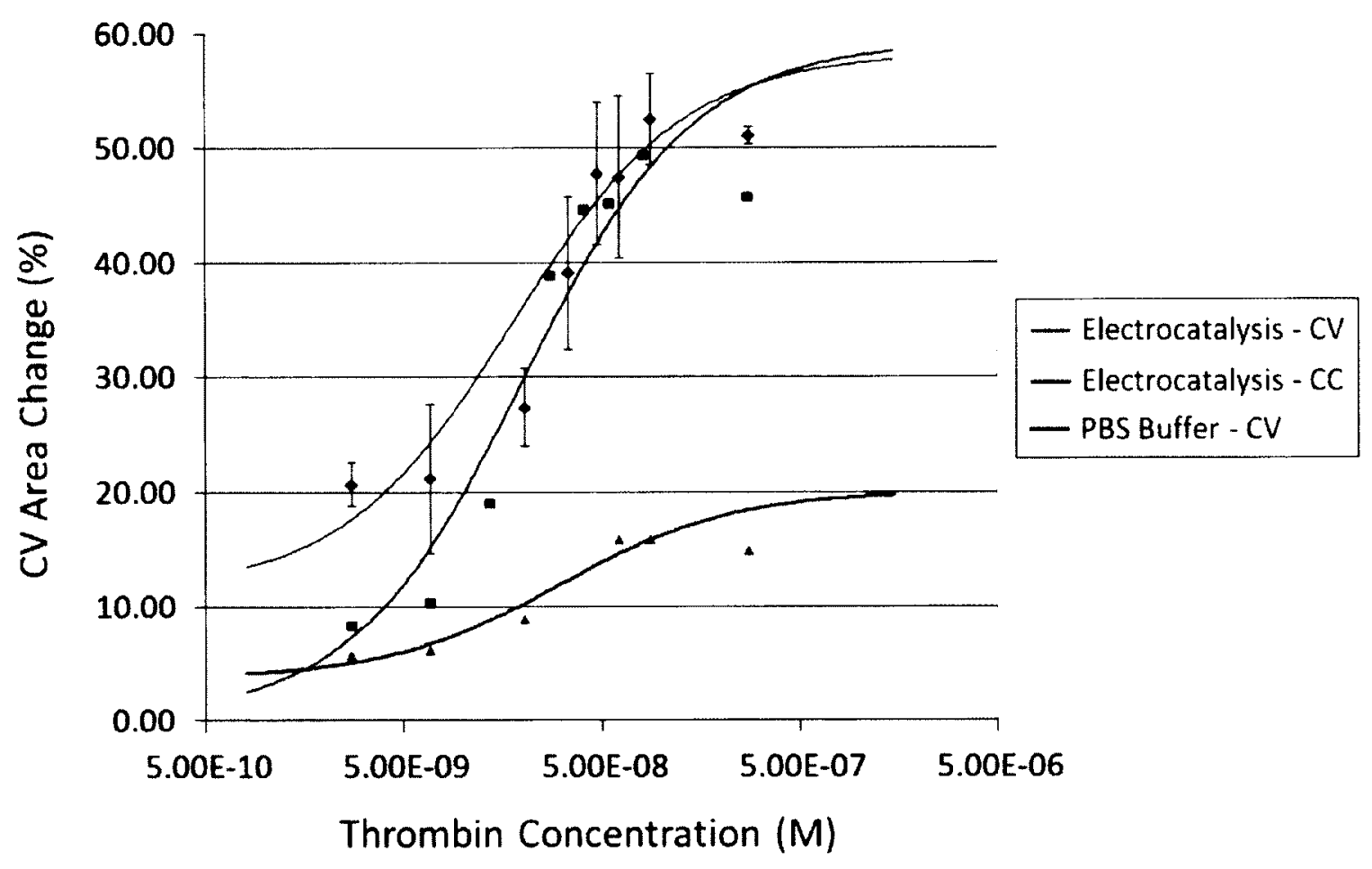

Figure 2-34: Sensor responses with $0.2 \mathrm{mM} \mathrm{K}_{3} \mathrm{Fe}(\mathrm{CN})_{6}$ for cyclic voltammetry (red) chronocoulometry (green), and without $\mathrm{K}_{3} \mathrm{Fe}(\mathrm{CN})_{6}$ (blue). Replication for the purpose of generating error bars for all curves proposed as future experiments.

Importantly, it could be seen that conducting the thrombin measurements in the presence of $\mathrm{K}_{3} \mathrm{Fe}(\mathrm{CN})_{6}$ did not alter the affinity of thrombin for the surface bound aptamers. The dissociation constants were determined to lie between $20-30 \mathrm{nM}$, in close agreement with literature values for surface bound thrombin aptamers ${ }^{58}$. Perhaps what was most promising for this signal-off architecture was the substantial increase in absolute signal. In the side-by-side comparison in Figure 2-31, it can be seen that the cathodic peak current values measured by cyclic voltammetry with electrocatalytic enhancement were nearly an order of magnitude higher than those with buffer only. This difference was translated when the cathodic peaks were integrated for Figure 2-32. From the slopes of the estimated linear portions of each sensor response curve, electrocatalytic 
enhancement appeared to induce a 7 -fold enhancement in sensitivity. Naturally, more replications of the thrombin sensing experiments would be required to give a fair assessment of limit of detection. However, the present results imply that with electrocatalytic enhancement the detection limit could be lowered by almost an order of magnitude.

Relative changes as measured by chronocoulometry were less pronounced than those measured by cyclic voltammetry, perhaps in part due to the uncertainty introduced when selecting the correct potential. It should be noted that attempts with chronocoulometry in the absence of $\mathrm{K}_{3} \mathrm{Fe}(\mathrm{CN})_{6}$ produced maximum currents which did not correlate with the thrombin concentration, to the point where a reasonable binding curve could not be fit for inclusion in Figure 2-34. In other words, only with electrocatalytic enhancement of sensitivity was chronocoulometric detection feasible. It should be noted that chronocoulometry has been applied in other electrochemical aptasensor architectures, such as the work of Shen et al. in the detection of adenosine monophosphate, and frequently in determinations of surface coverage of DNA modified electrodes $^{119-121}$. However, in these instances the DNA backbone is saturated electrostatically with a redox-active probe such as ruthenium hexammine, the signal does not arise from a single covalently attached probe. The present results suggest that electrocatalytic enhancement could make chronocoulometry viable for a wider variety of sensor architectures. 


\subsubsection{Sensor regeneration}

After exposure to the highest thrombin concentrations, it was found that rinsing the working electrodes with deionized water would not completely restore the signal to original values. A one minute soak in the guanidine hydrochloride followed by copious rinsing with deionized water was found to complete the restoration of the enhanced cathodic peak ${ }^{122}$. A minor increase in the charging current after regeneration was observed after regeneration, indicating potential wear on the MB-DNA monolayers.

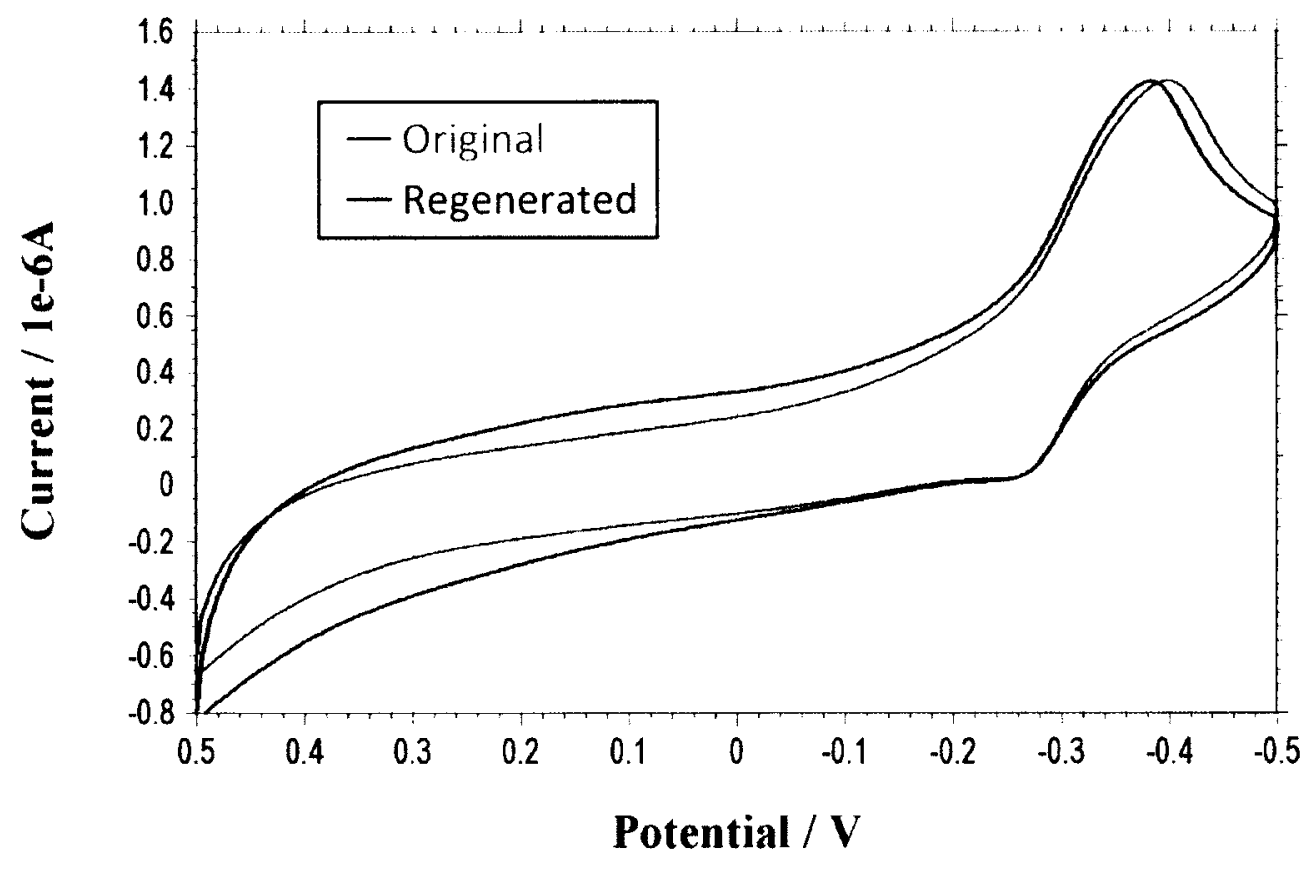

Figure 2-35: Regeneration of sensor surfaces via one minute soak in $8 \mathrm{M}$ guanidine hydrochloride. 


\subsection{Conclusions}

Methylene blue was successfully conjugated to an amino-modified thrombin aptamer via active ester coupling. After purification by means of ethanol precipitation and RP-HPLC and product confirmation by ESI-MS, the conjugate was deposited on a gold electrode to construct an electrochemical aptasensor for thrombin. The sensor functioned in accordance with literature expectations. In an effort to improve sensor performance, the methylene blue tag was used to electrocatalytically reduce $\mathrm{Fe}(\mathrm{CN})_{6}{ }^{3-}$, thereby greatly increasing the measured current in cyclic voltammetry experiments. Absolute signals were found to increase by approximately an order of magnitude. In addition to the consequent $\sim 7$-fold enhancement in sensitivity, increasing the absolute electrochemical signals should lessen the demand on sophistication of the equipment required for measurement ${ }^{73}$. The results could in principle be extended to any analyte for which an aptamer has been selected.

While the most basic sensor architecture possible was employed for this initial study of electrocatalytic enhancement, it may also be far more successful when applied to more complex architectures designed to bring about larger scale changes in the ability of the electrochemical tag to interact with the electrode. Signal-on architectures may particularly benefit. Nevertheless, the present findings may find practical application in the construction of future point of care devices with reduced equipment sensitivity. 
Chapter 3 - Development of aptamer-gadolinium conjugates as magnetic resonance imaging contrast agents 


\subsection{Statement of Contributions}

Project conception, synthesis design and purification of aptamer-gadolinium conjugates, and preliminary $300 \mathrm{MHz}$ NMR characterization by M.A. Beking. Xylenol orange test, transmetallation study, and $400 \mathrm{MHz}$ NMR characterization by E.D.Bernard. Low-field magnetic characterization by K. Rajamanickam.

\subsection{Introduction}

\subsubsection{Magnetic resonance imaging contrast agents (MRI-CAs)}

Magnetic resonance imaging (MRI) has grown to become one of the leading imaging modalities in modern medicine ${ }^{123}$. MRI is capable of generating high-resolution diagnostic images of the entire body without subjecting patients to ionizing radiation ${ }^{124}$. In essence, the technique exploits the variation in water content among different tissues to provide clinically relevant images with a high degree of spatial resolution ${ }^{125}$. Over one third of all MR image acquisitions include the use of contrasting agents to further improve diagnostic value ${ }^{126}$.

MRI-CAs amplify the naturally occurring differences in longitudinal relaxation time, $T_{1}$, and transverse relaxation time, $T_{2}$, of water protons in different tissue settings ${ }^{126}$. Paramagnetic gadolinium chelates are the most frequently employed for increasing signal intensity, and therefore diagnostic confidence, in $T_{1}$-weighted images ${ }^{127}$. Structures of two commonly used MRI-CAs with relevance to this work are 
presented in Figure 3-1. As of 2007 over 100 million administrations of gadoliniumbased MRI-CAs had been performed in the United States alone ${ }^{128}$.

i)

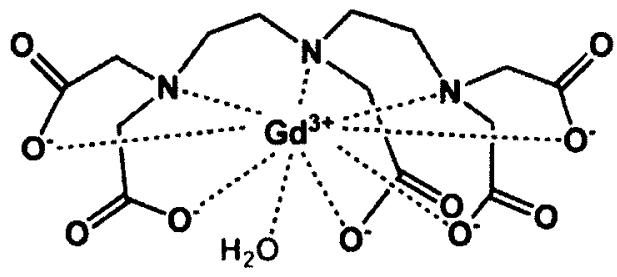

ii)

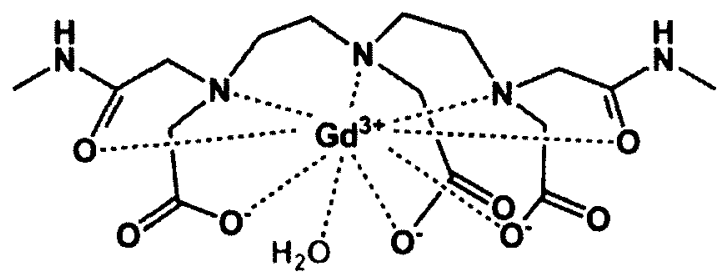

Figure 3-1: Structures of common gadolinium-based MRI-CAs (i) Magnevist (gadolinium diethylenetriaminepentaacetate) and (ii) Omniscan (a bisamide derivative). (adapted from Caravan et al.) ${ }^{129}$

The efficacy of an MRI-CA is dependent on its relaxivity, a measure of ability to increase the relaxation rate $\left(1 / T_{1}\right.$ or $\left.1 / T_{2}\right)$ of local water protons ${ }^{127}$. The relationship between relaxation rate, relaxivity, and gadolinium concentration is described by the following equation ${ }^{129}$ :

$$
\frac{1}{T_{i}}=\frac{1}{T_{i}^{o}}+r_{i}\left[G d^{3+}\right] ; i=1,2
$$

Thus, the $T_{1}$ relaxivity may be obtained from the slope of $1 / T_{1}$ vs. $\left[\mathrm{Gd}^{3+}\right]^{129}$. Several parameters influence the relaxivity of a gadolinium complex. These include the number of coordinated water molecules, the mean residence time of the coordinated water molecules, and the rotational correlation time of the complex ${ }^{130}$. By modifying the rotational correlation time, both molecular tumbling and solution viscosity can in turn influence relaxivity ${ }^{129,13 !}$. Relaxivity is also dependent on magnetic field strength. While higher relaxivities can be obtained at lower fields with careful optimization of water 
residence and rotational correlation times, there is value in characterization at higher field strengths looking to the future. A number of commercial MRI equipment manufacturers are investigating field strengths as high as $11.7 \mathrm{~T}(500 \mathrm{MHz})^{130}$. Vaughan et al. have acquired MR images from human volunteers at $9.4 \mathrm{~T}(400 \mathrm{MHz})$, and noted a doubling in the signal to noise ratio over images acquired at $4 \mathrm{~T}^{132}$.

\subsubsection{Nephrogenic systemic fibrosis (NSF)}

A fair overview of gadolinium-based MRI-CAs would not exclude a discussion of nephrogenic systemic fibrosis (NSF), a serious side effect observed exclusively in patients with advanced stages of kidney disease ${ }^{133}$. A causal link between the release of chelated gadolinium and NSF has been established, with a prevalence rate of $2.4 \%$ in dialysis patients, and $18 \%$ in the most advanced stage of kidney disease ${ }^{133,134}$. Consequently, a "box warning" was suggested for all gadolinium-based contrast agents by the food and drug administration in 2007, warning radiologists to avoid these agents when compromised renal function is suspected ${ }^{135}$. While the cases of NSF are certainly unfortunate, it would seem that an awareness of patient medical history can circumvent this issue. Contrast enhanced MRI remains extremely valuable in the tens of millions of scans performed annually, and current research in gadolinium-based agents features a heightened awareness of this safety concern ${ }^{123,127}$. 


\subsubsection{Active targeting with aptamers}

Due to the potential implications in early diagnosis and treatment of many conditions, there is an ongoing effort to develop improved MRI-CAs ${ }^{127,136}$. At an estimated $\$ 50-\$ 80$ per injection, the development high utility MRI-CAs is also compelling financially ${ }^{129}$. One method for improving the utility of gadolinium-based MRI-CAs has focused on the preparation of dendritic or polymeric structures to increase relaxivity ${ }^{137}$. While relaxivity per gadolinium centre remains similar, the agglomeration of a large number of paramagnetic centres leads to a higher molar relaxivity ${ }^{130.138}$.

Perhaps a more elegant approach is the preparation of modified chelates which actively accumulate in pathological tissue or that can become selectively involved in a particular biological process. Indeed, such functionalized MRI-CAs are prevalent in recent literature, with gadolinium chelates having been conjugated to steroids ${ }^{139}$, monosaccharides ${ }^{140}$, carbohydrates ${ }^{141}$, proteins ${ }^{138}$, and pharmaceuticals such as anticancer drugs $^{142}$ and coumarin derivatives ${ }^{143}$. Hines et al. reported the conjugation of diethylenetriaminepentaacetic acid (DTPA) to an oligonucleotide sequence for chelation of gadolinium and measured relaxivity changes upon binding to an antisense $\operatorname{target}^{131}$. Lu et al. reported the conjugation of a gadolinium chelate to an oligonucleotide complementary to a portion of the ATP aptamer ${ }^{144}$. A large streptavidin tag on the aptamer provided the molecular bulk necessary to generate pronounced differences in rotational correlation time upon ATP-binding induced displacement of the gadoliniumlabeled complement ${ }^{144}$. Direct conjugation of a gadolinium chelate to an aptamer, and where the target itself induces a relaxivity change, remains unreported ${ }^{145}$. 
Several aptamers have been used in optical-based imaging experiments ${ }^{145}$. An aptamer selected for recombinant integrin $\alpha v \beta 3$, an important cell adhesion protein with implications for tumor invasion and wound healing processes was fluorescently tagged for imaging ${ }^{145}$. Similar experiments with aptamers for angiogenin, prostate specific membrane antigen, and nucleolin have also been conducted ${ }^{145}$. The requirement for either histological processing or transparent samples precludes the direct extrapolation of these optical studies to clinical application ${ }^{140}$. Alternatively, MRI can be performed on living, opaque specimens, providing a more appropriate platform for applied study of aptamer behaviour in vivo.

With these goals in mind, previous undergraduate work detailed the basic synthesis of two aptamer-gadolinium conjugates, with structures shown in Figure 3-2 ${ }^{146}$.

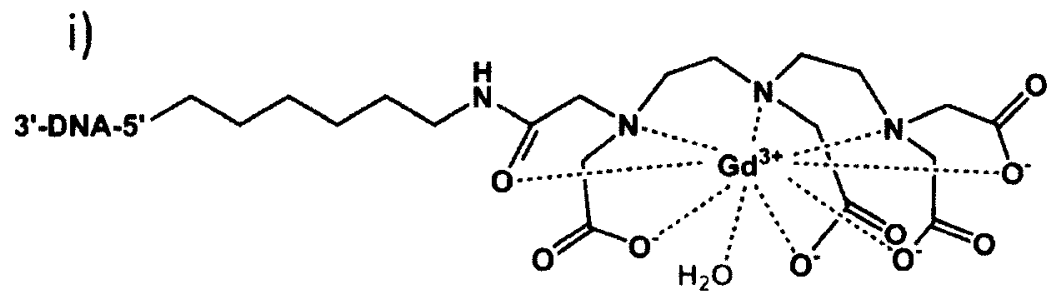

ii)

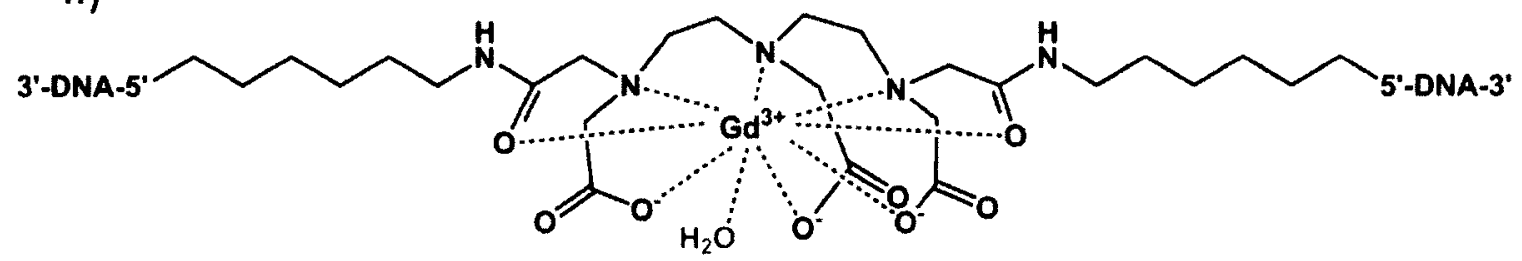

Figure 3-2: Structures of previously generated aptamer-gadolinium conjugates, (i) mono-substituted DTPA or 'single conjugate' (SC), (ii) di-substituted DTPA or 'double conjugate' $(\mathrm{DC})$. (DNA = thrombin aptamer) 


\subsubsection{DNA-templated synthesis}

Notably, the previously reported synthesis leads only to di-substituted conjugates featuring the same aptamer. While this would presumably increase active targeting capability, the prospect of multi-modal active targeting and some interesting therapeutic strategies would arise from an asymmetrically di-substituted conjugate. In the former suggestion, the incorporation of two different aptamer sequences could lead to increased selectivity in contrast agent accumulation. For the latter, one aptamer could be used for the purpose of active targeting, and the other could serve as part of a drug delivery or controlled release scheme $\mathrm{e}^{147.148}$. This combination of therapeutic and diagnostic value has prompted invention of the terms 'theragnostics' and 'theranostics' to describe similar approaches $^{147.149}$.

In order to design a synthetic pathway to such conjugates, concepts from the field of DNA-templated chemical reactions were applied ${ }^{150}$. For instance, the specific nature of DNA hybridization can be exploited to increase the effective molarity of reactants, not only greatly increasing the reaction rate, but also allowing control over which reactants are brought into proximity by manipulation of the DNA sequences ${ }^{151}$. This approach takes inspiration from nature, where biological processes such as DNA replication can be performed with concentrations of starting materials orders of magnitude below what is

required for laboratory imitation due to macromolecular templating ${ }^{151}$. In an interesting deviation from the more commonly used DNA hybridization approach, Heemstra and coworkers recently applied this principle to ligate split fragments of the cocaine aptamer $^{152}$. Ligation yields were negligible in the absence of cocaine target or with the 
introduction of a single mutation in the aptamer sequence ${ }^{152}$. Liu and coworkers have also proposed interesting template architectures, with Figure 3-3 presenting examples ${ }^{153,154}$.

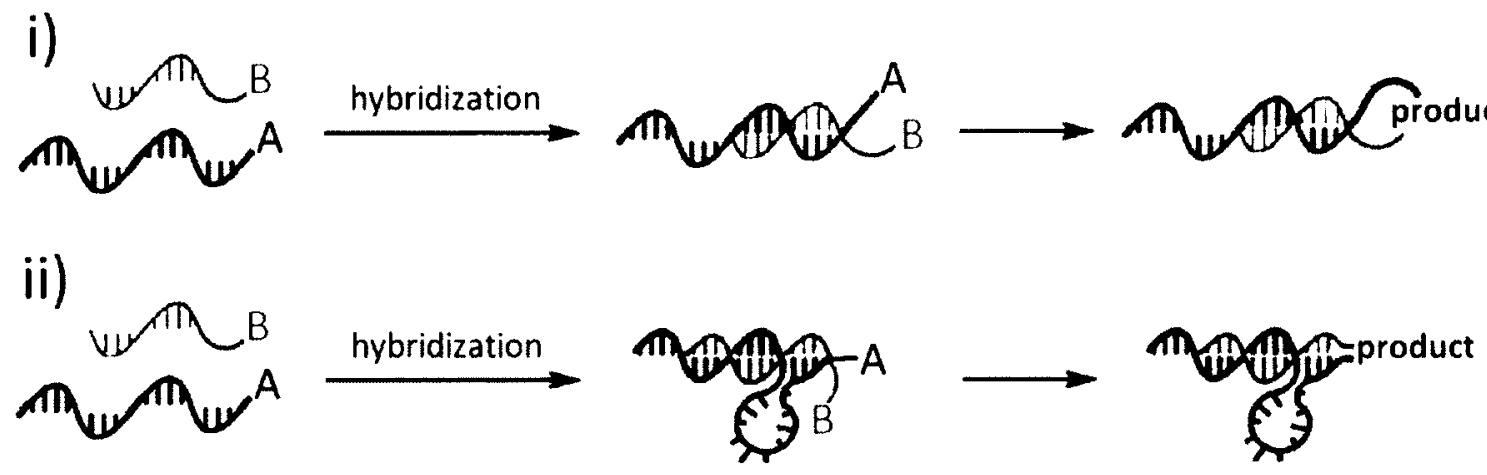

Figure 3-3: DNA-templated synthesis architectures for increasing the effective molarity of reagents $A$ and B: (i) linear architecture, (ii) $\Omega$ architecture. (adapted from Rozenman et al.) ${ }^{153}$

\subsubsection{Project objectives}

The first objective of this project was to introduce improvements to the synthesis of aptamer-gadolinium conjugates, specifically for the purpose of increasing yields of disubstituted conjugates. Next, magnetic characterization and stability assessments would demonstrate the value of the mono-conjugates as MRI-CAs. Finally, a re-imagined synthetic route which could produce asymmetrically di-substituted conjugates based on the established principles of DNA-templated chemical reactions will be introduced. 


\subsection{Experimental}

\subsubsection{Materials}

Diethylenetriaminepentaacetic acid dianhydride (DTPA-DA) was obtained from Sigma Aldrich. Disuccinimidyl suberate (DSS) was obtained from Fisher Scientific. Millipore Milli-Q deionized water at $18 \mathrm{M} \Omega$ was used in all experiments. Deuterium oxide was obtained from $\mathrm{CDN}$ Isotopes. Oligonucleotide synthesis reagents, phosphoramidites, and modifiers were purchased from Glen Research. Support columns and acetonitrile were purchased from BioAutomation. Ultra High Purity 5.0 argon was obtained from Praxair. All other chemicals and solvents were of reagent grade or higher, and used as received.

\subsubsection{Synthesis of aptamer-gadolinium conjugates}

The basic synthesis of aptamer-gadolinium conjugates has been previously described $^{146}$. The synthesis was modified from the method of Hnatowich et al. for radiolabeling proteins ${ }^{155}$. DTPA-DA was used for conjugation to amino-modified thrombin aptamer by the mechanism proposed in Figure 3-3. For this reaction, DTPA-DA dissolved in anhydrous DMSO was added to CPG bound, amino-modified thrombin aptamer. The procedures that follow were adaptations made during the present work to encourage the formation of di-substituted DTPA conjugates. 


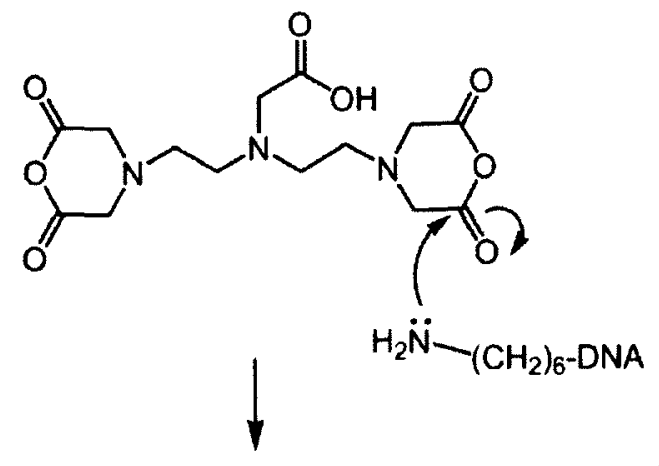

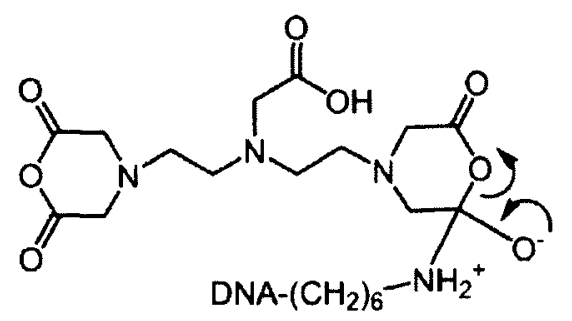<smiles>C#CC</smiles><smiles>O=C([O-])CN(CCN(CC(=O)[O-])CC(=O)NCCCCCCCCCCC(=O)O)CC(=O)O</smiles><smiles>C#C</smiles>

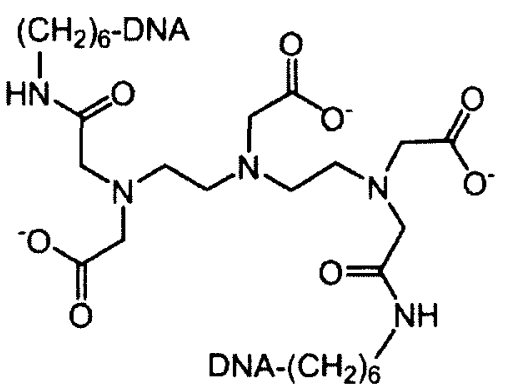

Figure 3-4: The conjugation of DTPA-dianhydride to amino-modified DNA, followed by (i) hydrolysis of the second anhydride to form a mono-substituted conjugate, or (ii) reaction with a second equivalent of amino-modified DNA at the second anhydride to form a di-substituted conjugate.

\subsubsection{Oligonucleotide synthesis and modified DTPA conjugation reactions}

Oligonucleotides were synthesized via standard phosphoramidite chemistry as previously described. Syntheses were carried out at the $1 \mu \mathrm{mol}$ scale with $500 \AA$ pore size columns and an extension of the 5'-modifier coupling time by 60 seconds. The following sequences were produced, with the synthesizer set to leave the 5'-amino modification protected: 


\section{Thrombin aptamer: $\mathrm{H}_{2} \mathrm{~N}-\left(\mathrm{CH}_{2}\right)_{6}-5^{\prime}-\mathrm{GGT}$ TGG TGT GGT TGG-3'}

15T - thrombin aptamer: $\mathrm{H}_{2} \mathrm{~N}-\left(\mathrm{CH}_{2}\right)_{6}-5^{\prime}-$ TTT TTT TTT TTT TTT GGT TGG TGT GGT TGG-3'

30T - thrombin aptamer: $\mathrm{H}_{2} \mathrm{~N}-\left(\mathrm{CH}_{2}\right)_{6}-5^{\prime}-$ TTT TTT TTT TTT TTT TTT TTT TTT TTT TTT GGT TGG TGT GGT TGG-3'

The columns were treated post-synthesis with $1 \mathrm{~mL}$ of $10 \%$ diethylamine in anhydrous acetonitrile, washed with two column volumes of acetonitrile, and dried with argon. The CPG support beads were divided equally, with one half set aside at $-20^{\circ} \mathrm{C}$, while oligonucleotides from the other half were cleaved and deprotected in $1 \mathrm{~mL}$ of aqueous ammonia at room temperature for 24 hours. The CPG beads were removed by filtration with $0.22 \mu \mathrm{m}$ nylon microcentrifuge filter tubes, and the filtrates were concentrated in vacuo at room temperature. The 4,4'-dimethoxy-4' '-methylsulfonyltrityl (DMS $(\mathrm{O}) \mathrm{MT}$ ) group protecting the amino modification was removed by resuspending the pellet in $2 \%$ dichloroacetic acid and incubating for 5 minutes. Ethanol precipitation, described in Section 2.2.2.3, was used to isolate the deblocked oligonucleotides.

The remaining half of the CPG support beads were treated with two column volumes of $3 \%$ dichloroacetic acid in dichloromethane. The beads were transferred to a microcentrifuge tube and suspended in anhydrous DMSO. DTPA-DA $(5.4 \mathrm{mg}, 15 \mu \mathrm{mol})$ was added, and the tube was shaken for 4 hours. DTPA-DA was either added as the entire quantity in a single step, or titrated into the microcentrifuge in small aliquots with gentle shaking between additions, as described in Table 3-1. 
Table 3-1: The titration method of encouraging DNA-DTPA-DNA conjugates.

\begin{tabular}{|l|l|l|l|}
\hline \multicolumn{1}{|c|}{$\begin{array}{c}\text { Addition } \\
\text { Number }\end{array}$} & \multicolumn{1}{|c|}{$\begin{array}{c}\text { DTPA-DA } \\
\text { added }\end{array}$} & Shaking time & $\begin{array}{c}\text { Cumulative } \\
\text { DTPA-DA }\end{array}$ \\
\hline $1-6$ & $0.025 \mu \mathrm{mol}$ & 10 minutes & $0.15 \mu \mathrm{mol}$ \\
\hline $7-12$ & $0.05 \mu \mathrm{mol}$ & 5 minutes & $0.45 \mu \mathrm{mol}$ \\
\hline $13-18$ & $1 \mu \mathrm{mol}$ & 5 minutes & $6.45 \mu \mathrm{mol}$ \\
\hline $19-21$ & $2 \mu \mathrm{mol}$ & 5 minutes & $12.45 \mu \mathrm{mol}$ \\
\hline Remainder & $2.55 \mu \mathrm{mol}$ & Overnight & $15 \mu \mathrm{mol}$ \\
\hline
\end{tabular}

The supernatant was discarded, and the beads were rinsed with DMSO. Next, the cleaved half of the synthesized oligonucleotides, with solubility in DMSO aided by cetyltrimethylammonium bromide (CTAB), was added and the mixture was shaken for 24 hours. The beads were further rinsed with DMSO, treated with $1 \mathrm{~mL}$ of aqueous ammonia for 24 hours, and filtered. Concentration of the filtrate in vacuo yielded the crude products.

\subsubsection{Purification of DNA-DTPA conjugates}

Conjugates were purified by poly(acrylamide) gel electrophoresis (PAGE) by the method previously described in Section 2.2.2.5. 


\subsubsection{Chelation of gadolinium(III)}

Following purification, conjugates were combined with $\mathrm{GdCl}_{3}$ in chelation buffer $(50 \mathrm{mM}$ tris, $140 \mathrm{mM} \mathrm{NaCl}, \mathrm{pH} 6)$, so as to produce a 1:1 molar ratio of $\mathrm{Gd}^{3+}$ to aptamerDTPA. Conjugates were shaken overnight, and desalted with an Amicon Ultra microcentrifugal filter. The filtrate was evaluated for nonchelated $\mathrm{Gd}^{3+}$ content by a xylenol orange test. Briefly, four solutions containing an equivalent amount of xylenol orange were prepared. Two of these received known amounts of $\mathrm{Gd}^{3+}$, one received the above filtrate, and the last served as a blank. Gadolinium concentration dependent colour changes were detected by UV-Vis. This process of incubation with $\mathrm{Gd}^{3+}$, desalting, and measurement was repeated until $\mathrm{Gd}^{3+}$ measured in the filtrate reached a stable value, indicative of no additional uptake into aptamer-DTPA. Conjugate samples of approximately $1 \mathrm{nmol}$ were sent to Novatia for ESI-MS.

\subsubsection{Transmetallation study}

Prepared aptamer-gadolinium conjugates were studied for stability to transmetallation by $\mathrm{Zn}^{2+}$ using the method of Muller and coworkers ${ }^{156}$. The relaxation rate $\left(1 / T_{1}\right)$ of a solution of conjugates was measured at $9.4 \mathrm{~T}$ in deuterated phosphate buffer. Then, an equimolar amount of $\mathrm{Zn}^{2+}$ was added to the NMR tube and the relaxation rate was remeasured periodically over three days. 


\subsubsection{Magnetic characterization}

Preliminary measurements of relaxivity were performed by determing $T_{1}$ relaxation times for a range of mono-conjugated thrombin aptamer concentrations with a $300 \mathrm{MHz}(7.0 \mathrm{~T})$ Bruker NMR spectrometer and a standard inversion recovery pulse program. Measurements at these same concentrations were repeated with the addition of equimolar amounts of thrombin, and again with bovine serum albumin (BSA) as a control.

Characterization of the complete set of mono-conjugated aptamers, and aptamer conjugates with 15 poly(T) and 30 poly(T) spacer sequences was performed at $9.4 \mathrm{~T}$ $(400 \mathrm{MHz})$. The conjugates were characterized alone, with equimolar amounts of thrombin, and with equimolar amounts of pepsin as a control. Additionally, poly(A) sequences complementary to the poly(T) linkers were introduced in a separate set of experiments. Magnetic characterization of mono-conjugated thrombin aptamer with a clinical 1.5T instrument was also performed at the Civic Hospital, Ottawa.

\subsubsection{DNA templated synthesis}

The following oligonucleotide sequences were synthesized by standard phosphoramidite chemistry, cleaved from the solid supports and deprotected as previously described. ESI-MS was used to confirm the products. 
E10: 5'-AAT TCG TAC C-3'- $\mathrm{CH}_{2}-\mathrm{CH}\left(\mathrm{CH}_{2} \mathrm{OH}\right)-\left(\mathrm{CH}_{2}\right)_{4}-\mathrm{NH}_{2}$

E20: 5'-TCC CGA GTC GAA TTC GTA CC-3'- $\mathrm{CH}_{2}-\mathrm{CH}\left(\mathrm{CH}_{2} \mathrm{OH}\right)-\left(\mathrm{CH}_{2}\right)_{4}-\mathrm{NH}_{2}$

T30: $\mathrm{H}_{2} \mathrm{~N}-\left(\mathrm{CH}_{2}\right)_{6}-5$ '-GGT ACG AAT TCG ACT CGG GAA TAC CAC CCT T-3'

The DMS $(\mathrm{O})$ MT protecting group was removed from T30 as described in Section 3.2.2.1. Melting studies were performed to ensure the desired hybridization behaviour. Briefly, equimolar quantities of E10 or E20 and T30 in $70 \mathrm{mM} \mathrm{NaCl}$ were combined in a cuvette with a miniature stir bar. The temperature was increased at a rate of $1^{\circ} \mathrm{C}$ per minute, with UV-Visible spectra recorded at one minute intervals.

For DNA-templated synthesis, $2 \mathrm{nmol}$ of E10 or E20 was combined with $2 \mathrm{nmol}$ of $\mathrm{T} 30$, and the mixtures were heated to $70^{\circ} \mathrm{C}$ then allowed to cool. Conjugation reactions were performed with both DSS and DTPA-DA in parallel. DSS and DTPA-DA were added in 50 -fold excesses, dissolved in either $0.1 \mathrm{M}$ sodium phosphate $\mathrm{pH} 8$ or $\mathrm{N}, \mathrm{N}$ DMF. The final organic to aqueous solvent ratio in the case of N,N-DMF reactions was 95:5. Reaction mixtures were shaken at room temperature overnight, then centrifuged at maximum speed for 5 minutes. Supernatants were removed and crude products were isolated by ethanol precipitation. As an alternative in a separate trial, reaction mixtures were not centrifuged, but were rather simply concentrated in vacuo to yield crude products. After characterization by PAGE, bands of interest were excised, eluted from the gel, and sent to Novatia for ESI-MS analysis. 


\subsection{Results and Discussion}

\subsubsection{Synthesis of aptamer-gadolinium conjugates}

Multiple improvements were made to the synthesis of aptamer-gadolinium conjugates in comparison to the original description ${ }^{146}$. The originally reported synthesis featured the simple addition of DTPA-DA to support bound amino-modified oligonucleotides and allowing the reaction to proceed. After observation of both monoand di-substituted conjugation products, it was apparent that the second anhydride could react with a nearby support bound amino-modified oligonucleotide. Alternatively, the second anhydride would be subjected to hydrolysis during work up. These are depicted as reactions i) and ii) in Figure 3-5 respectively. Reaction i) was clearly a minor pathway under the original reaction conditions, indicated by an approximate 100:1 ratio of monoto di-substituted products.

In the present work, two alterations to the synthesis were made in an effort to increase the resultant proportion of di-substituted conjugates. First, it was considered that subjecting the support bound oligonucleotides to the entire excess of DTPA-DA immediately would result in more complete labeling. While desirable for mono-conjugate production, this left little potential for reaction i) to occur.

Reaction i) was reminiscent of a synthesis performed by Sleiman and coworkers, who prepared ruthenium complex linked oligonucleotides directly on CPG supports ${ }^{157}$. Specifically, a bisphosphoramidite was used for conjugation of adjacent hydroxylterminated oligonucleotides, while the present reaction i) features a dianhydride for conjugation of adjacent amino-terminated oligonucleotides ${ }^{157}$. Based on this precedent, it 
was hypothesized that titrating DTPA-DA into the reaction mixture in small quantities, sufficient to label only a fraction of the support bound oligonucleotides with each addition, would increase the probability for reaction i) to take place. Indeed, the lowest ratio of mono- to di-substituted conjugates observed with this titration method was $7: 1$, a considerable improvement over the original synthesis. Competition from hydrolysis would have been the main factor opposing the di-conjugates.
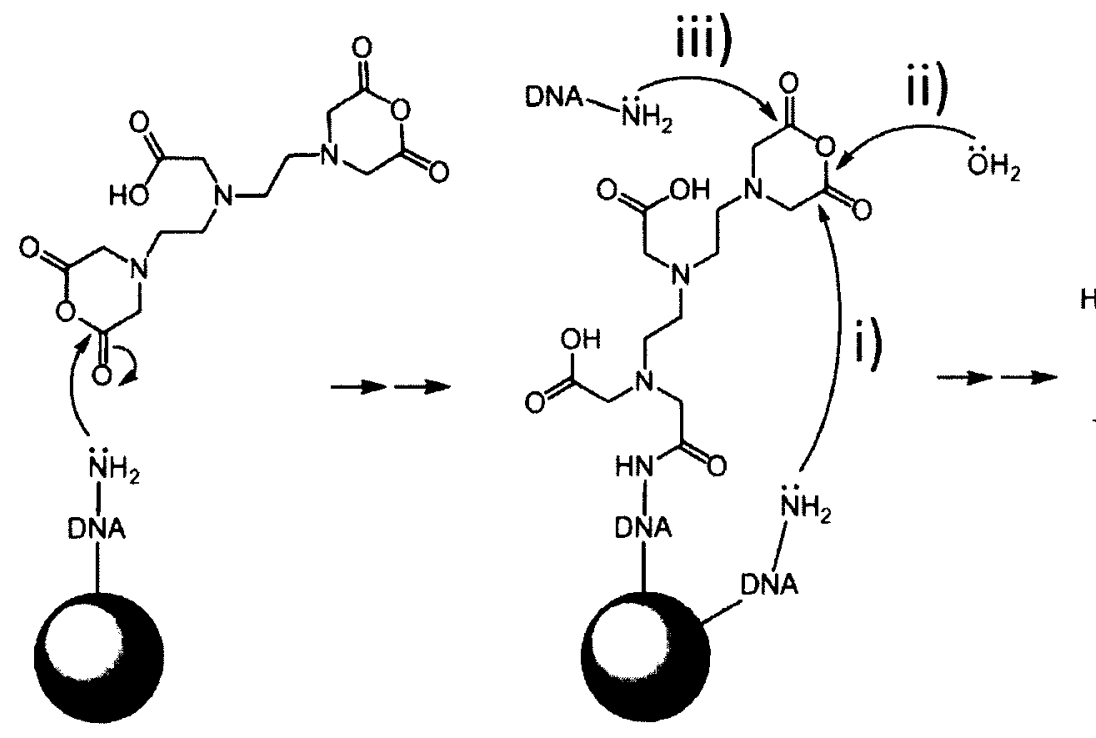<smiles>O=C(O)CN(CCN(CCN(CC(=O)O)CC(=O)NO)CC(=O)NO)CC(=O)O</smiles>

Figure 3-5: Potential reactions at the second dianhydride after initial conjugation of DTPA-DA to support bound $\mathrm{NH}_{2}$-DNA: i) Reaction with a nearby $\mathbf{N H}_{2}$-DNA yielding a di-conjugate, ii) Hydrolysis yielding a mono-conjugate, iii) Addition of a second equivalent of $\mathrm{NH}_{2}$-DNA to yield a di-conjugate.

As an additional strategy, a second equivalent of cleaved, deprotected, and deblocked amino-modified DNA was added to the reaction mixture shortly after the initial DTPA-DA conjugation. The consequent yield of di-substituted conjugates would be increased if reaction (iii) from Figure 3-5 were to proceed more quickly than hydrolysis. This was attempted following both the addition of the entire excess of DTPA- 
DA and the slow addition of DTPA-DA over time. Table 3-2 summarizes the relative performance of each method. The ratios were determined from comparative absorbance within a poly(acrylamide) gel as determined by the Alpha Imager software.

Table 3-2: Effectiveness of adapted methods toward the production of di-substituted aptamer-DTPA conjugates.

\begin{tabular}{|l|c|c|c|c|}
\hline Method & $\begin{array}{c}\text { Original } \\
(4908)\end{array}$ & Titration & $\begin{array}{c}\text { Additional } \\
\mathrm{NH}_{2} \text {-DNA }\end{array}$ & $\begin{array}{c}\text { Titration }+ \\
\text { Additional } \\
\mathrm{NH}_{2} \text {-DNA }\end{array}$ \\
\hline $\begin{array}{l}\text { Lowest ratio of mono- } \\
\text { to di-substituted } \\
\text { conjugate observed }\end{array}$ & $100: 1$ & $7: 1$ & $3: 1$ & $7: 1$ \\
\hline
\end{tabular}

The lowest ratio of mono- to di-substituted conjugates, 3:1, was observed when additional $\mathrm{NH}_{2}$-DNA was added after initial DTPA-DA conjugation was performed in a single step. Hydrolysis was most likely a limiting factor in the hybrid approach, where a 7:1 ratio was again the lowest observed. Figure 3-6 presents a typical separation of monoand di-substituted conjugates, where the relative absorbance of the lower to the upper band was used to compute the ratio of mono- to di-substituted conjugates. 


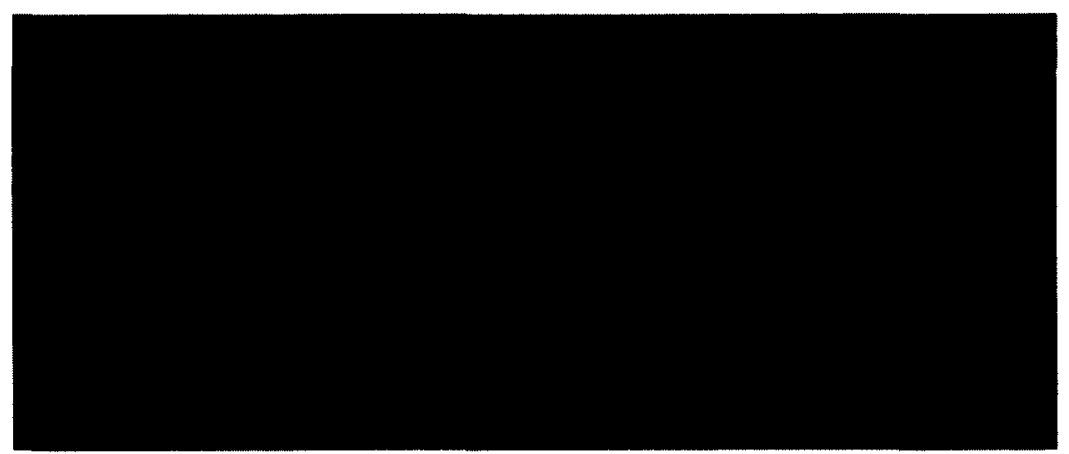

Figure 3-6: Poly(acrylamide) gel separation of mono- and di-substituted aptamerDTPA conjugates. Left lanes correspond to conjugated prepared with the thrombin aptamer alone, right lanes to the $15 \mathrm{~T}$-thrombin aptamer.

Following elution from the poly(acrylamide) gels, $\mathrm{Gd}^{3+}$ was introduced to the aptamer-DTPA conjugates for chelation. Large molar excesses of $\mathrm{Gd}^{3+}$ were avoided due to the potential for electrostatic interaction with the negatively charged DNA backbone. For the same reason, a fairly high ionic strength chelation buffer with no components that could form strong complexes with DTPA was employed ${ }^{158}$. To quantify free $\mathrm{Gd}^{3+}$ after the chelation reaction, a colorimetric test based on the interaction of xylenol orange with lanthanides was devised ${ }^{159}$. Conjugates were re-incubated with additional $\mathrm{Gd}^{3+}$ until the proportion of aptamer-DTPA $\left(\mathrm{Gd}^{3+}\right)$ in the retentate was calculated to be greater than $90 \%$. 


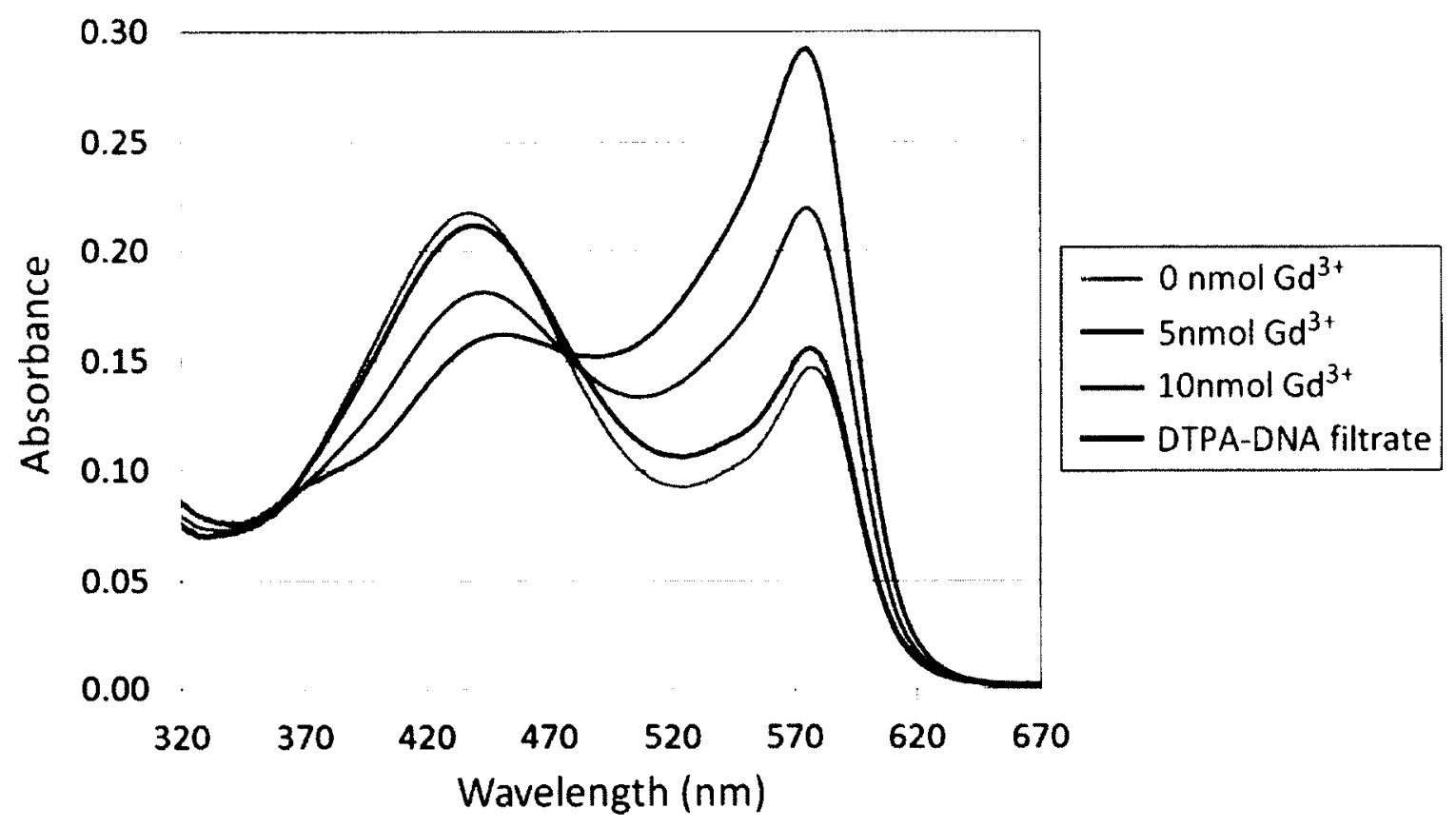

Figure 3-7: UV-Vis spectra from the xylenol orange test for $\mathrm{Gd}^{3+}$. Absorbance at $\sim 570 \mathrm{~nm}$ was used to determine $\mathrm{Gd}^{3+}$ present in the aptamer-DTPA $\left(\mathrm{Gd}^{3+}\right)$ filtrate.

\subsubsection{Transmetallation Study}

One of the principle considerations with the use of gadolinium based MRI-CAs is the potential for release of the toxic $\mathrm{Gd}^{3+}$ ion by in vivo exchange with endogenous cations ${ }^{147}$. While ubiquitous cations such as $\mathrm{Na}^{+}, \mathrm{K}^{+}$and $\mathrm{Ca}^{2+}$ only form weak complexes and are thus thermodynamically unfavoured, $\mathrm{Zn}^{2+}, \mathrm{Cu}^{2+}$, and $\mathrm{Fe}^{3+}$ and may take the place of $\mathrm{Gd}^{3+}$ within chelators by a process termed transmetallation ${ }^{156,158} \cdot \mathrm{Zn}^{2+}$ is considered the most problematic, as the serum concentration of $\mathrm{Cu}^{2+}$ is much lower, and $\mathrm{Fe}^{3+}$ is tightly controlled by transferrin and unlikely to participate in transmetallation ${ }^{158}$.

To determine the susceptibility of the present conjugates to transmetallation, a straightforward method proposed by Muller and coworkers was applied ${ }^{156}$. For this 
experiment, an equimolar amount of $\mathrm{Zn}^{2+}$ was introduced to a solution of conjugate with a known relaxation rate in phosphate buffer. Liberated $\mathrm{Gd}^{3+}$ precipitates rapidly with $\mathrm{PO}_{4}{ }^{3-}$ present in the buffer, to form insoluble $\mathrm{GdPO}_{4}\left(\mathrm{~K}_{\mathrm{sp}} \approx 10^{-22} \mathrm{M}^{2}\right)$. A decrease in the relaxation rate can be correlated to the loss of the paramagnetic contribution from $\mathrm{Gd}^{3+}$ as it is converted to $\mathrm{GdPO}_{4}$ via the transmetallation process ${ }^{160}$. The use of a 1:1 ratio of $\mathrm{Zn}^{2+}$ to aptamer-gadolinium conjugate was considered quite conservative, as the in vivo concentration of $\mathrm{Zn}^{2+}$ would presumably be much lower than the target-accumulated conjugate $^{156}$. The conservative nature of this study was valuable in consideration of the potential additive effect of transmetallation by a number of different cations, not only $\mathrm{Zn}^{2+}$. Figure 3-8 presents the normalized relaxivity changes over time.

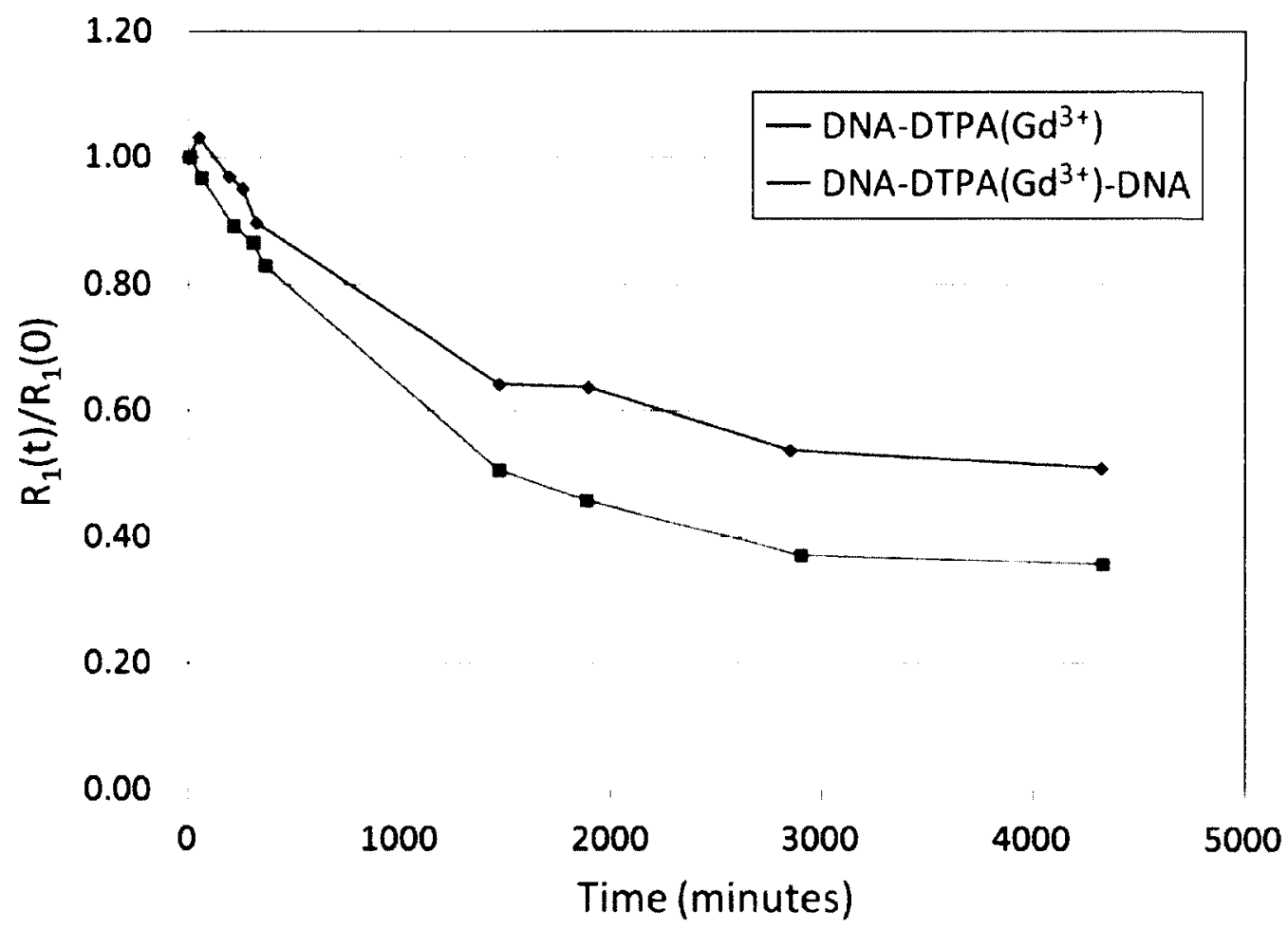

Figure 3-8: Decay in the measured relaxivity of aptamer-DTPA $\left(\mathbf{G d}^{3+}\right)$ resulting from transmetallation by $\mathrm{Zn}^{2+}$ and precipitation of $\mathrm{Gd}^{3+}$ by $\mathrm{PO}_{4}^{-}$. 
Firstly, the relaxivity of aptamer-DTPA $\left(\mathrm{Gd}^{3+}\right)$ was found to decay to about half of the intial value over approximately three days, which is comparable to literature data for $\operatorname{DTPA}\left(\mathrm{Gd}^{3+}\right)$ alone ${ }^{156}$. This was encouraging, as the substitution of one carboxylate for an amide as a consequence of the conjugation reaction was expected to moderately increase transmetallation ${ }^{158}$. The di-substituted aptamer-DTPA $\left(\mathrm{Gd}^{3+}\right)$-aptamer conjugate measured here was found to be somewhat less resistant to transmetallation over time, a likely consequence of the substitution of a second carboxylate. Due to rapid clearances from the body, perhaps more important from a safety perspective than the thermodynamically dictated value at which $R_{l}(t) / R_{l}(0)$ settles is the kinetic index, or the time taken for $R_{l}(t) / R_{l}(0)$ to reach a value of $0.80^{156}$. The kinetic indices measured here for mono- and di-substituted conjugates, approximately 11 hours and 6 hours respectively, lie well beyond the expected clearance times for either the gadolinium chelate or an aptamer. Gadolinium chelates are more rapidly eliminated, with half lives of less than 2 hours and aptamers more rapidly still, eliminated in minutes or less when unmodified ${ }^{15,37,130}$. Future in vivo studies would indicate whether the pharmacokinetics of the conjugates differ significantly.

\subsubsection{Magnetic characterization}

While synthesis strategies were adapted for the production of di-substituted aptamer-DTPA $\left(\mathrm{Gd}^{3+}\right)$ conjugates, yields of mono-substituted conjugates were sufficient to begin magnetic characterization. Tables 3-3 and 3-4 contain the results of preliminary characterization performed at $300 \mathrm{MHz}(7.0 \mathrm{~T})$ and characterization of the full set of mono-substituted conjugates performed at $400 \mathrm{MHz}(9.4 \mathrm{~T})$ respectively. 
Table 3-3: Preliminary characterization of thrombin aptamer-DTPA(Gd $\left(\mathrm{Gd}^{3+}\right)$ conjugates at $300 \mathrm{MHz}$.

\begin{tabular}{|c|c|c|c|}
\hline Sample & $\begin{array}{c}\text { Relaxivity } \\
\left(\mathbf{m M}^{-1} \mathbf{s}^{-1}\right)\end{array}$ & Protein & $\begin{array}{c}\text { Relaxivity } \\
\text { enhancement (\%) }\end{array}$ \\
\hline \multicolumn{4}{|l|}{ Thrombin aptamer-DTPA $\left(\mathrm{Gd}^{3+}\right)(\mathbf{S C})$} \\
\hline SC & 2.58 & -- & -- \\
\hline SC & 2.44 & BSA (control) & -- \\
\hline SC & 3.00 & Thrombin & $22.9^{\text {(a) }}$ \\
\hline
\end{tabular}

(a) Measured relative to BSA control

Table 3-4: Characterization of thrombin aptamer-DTPA(Gd $\left(\mathrm{Gd}^{3+}\right)$ and thrombin aptamer-poly(T)-DTPA $\left(\mathrm{Gd}^{3+}\right)$ conjugates at $400 \mathrm{MHz}$.

\begin{tabular}{|l|c|c|c|}
\hline \multicolumn{1}{|c|}{ Sample } & $\begin{array}{c}\text { Relaxivity } \\
\left(\mathbf{m M}^{-1} \mathbf{s}^{-1}\right)\end{array}$ & Protein & $\begin{array}{c}\text { Relaxivity } \\
\text { enhancement (\%) }\end{array}$ \\
\hline DTPA(Gd $\left.{ }^{3+}\right)$ & $5.72 \pm 0.05$ & -- & -- \\
\hline \multicolumn{4}{|c|}{} \\
\hline Thrombin aptamer-DTPA $\left(\mathrm{Gd}^{3+}\right)(\mathbf{S C})$
\end{tabular}

(a) Measured relative to DTPA $\left(\mathrm{Gd}^{3+}\right)$

(b) Measured relative to corresponding pepsin control experiment 
Mathematical descriptions of the interaction between the factors of field strength, rotational correlation time, and water residence time may be found in the SolomonBloembergen-Morgan equations, which have complexity and data requirements beyond the scope of this project ${ }^{161}$. However, these parameters can be discussed qualitatively to rationalize the observations, as is common in the contemporary literature when novel MRI-CAs are introduced ${ }^{137.158}$. Rotational correlation time, whether slowed by aptamertarget binding, formation of more rigid poly(A)-poly(T) hybrids, or a simple change in solution viscosity, would increase the measured relaxivity. On the other hand, a reduced water exchange rate by increased crowding around the aptamer-gadolinium conjugate would lower measured relaxivity. From Table 3-4, it can be seen that these offsetting effects of slowed rotational correlation time and hindered water exchange rate affected absolute relaxivity and the relaxivity enhancements somewhat non-systematically across different linker lengths and rigidities. For instance, rigidifying a poly( $\mathrm{T})$ linker by hybridization with a poly(A) sequence improved the relaxivity enhancement for a $15 \mathrm{~T}$ linker, but reduced the enhancement for a 30T linker. 


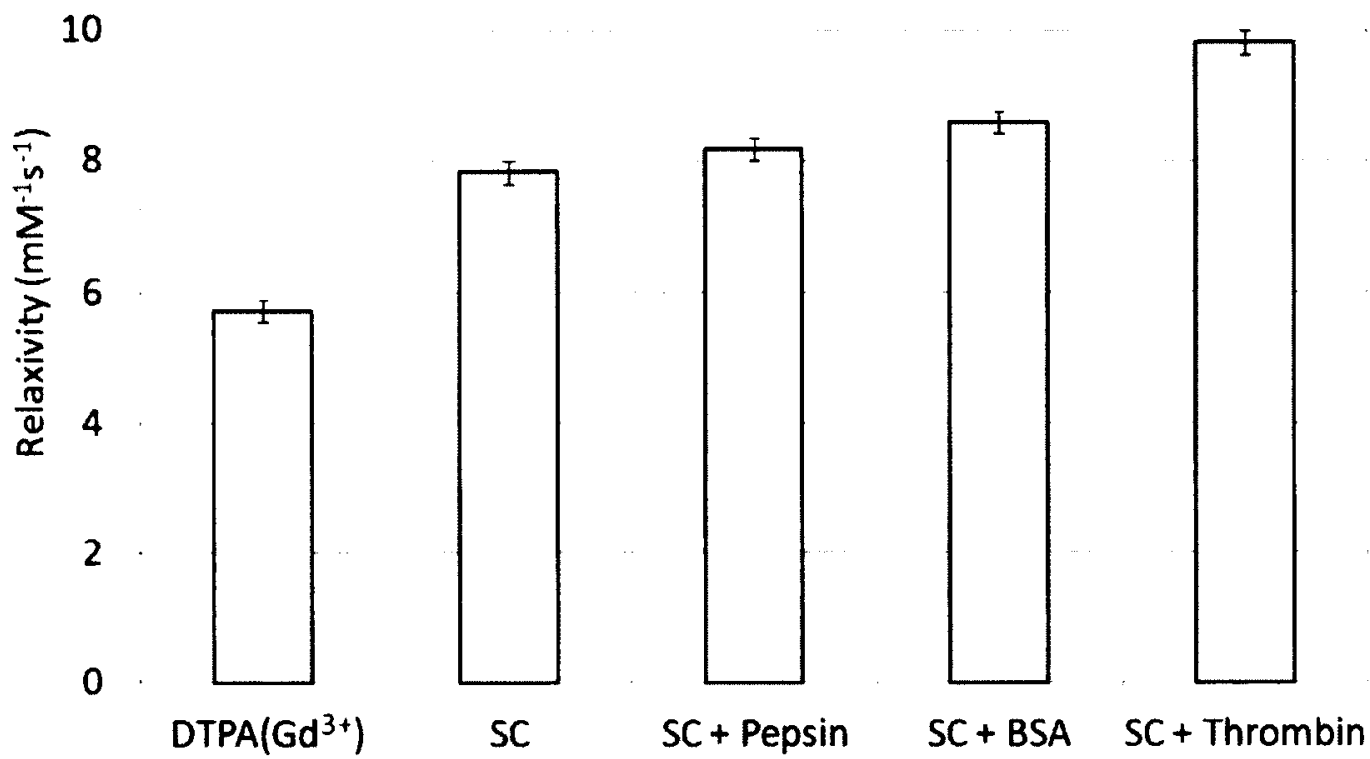

Figure 3-9: Low-field magnetic characterization of thrombin aptamer-DTPA(Gd $\left.{ }^{3+}\right)$ conjugates performed with a clinical MR instrument.

Measurements of relaxivity within a clinical $1.5 \mathrm{~T}$ instrument were also promising, and more consistent with literature-based expectations. A significant increase in relaxivity was observed for $\mathrm{SC}$ due to a slowed rotational correlation time in comparison to DTPA $\left(\mathrm{Gd}^{3+}\right)$ alone. Slight increases with the control proteins may be rationalized by small changes in solution viscosity, and the significant improvement upon introduction of thrombin target a consequence of a significantly slowed rotational correlation time ${ }^{129}$.

The more compelling value of aptamer-gadolinium conjugates as MRI-CAs may rest with the prospect of active targeting and the selective accumulation of gadolinium centres in pathological tissues. Nevertheless, the added benefit of relaxivity enhancements as high as $70 \%$ upon target binding would also be valuable. Thus, aptamer-gadolinium conjugates may represent a two-pronged approach to improved 
MRI-CAs, featuring both relaxivity improvements as shown here and the suspected improvement in biodistribution ${ }^{37.145}$.

\subsubsection{DNA-templated synthesis}

In order to produce asymmetrically di-substituted conjugates, the DNA-templated synthesis architectures of Liu and coworkers were employed ${ }^{153}$. Specifically, the linear architecture presented as Figure 3-3 i) was used, with both 10 and 20 nt duplex lengths ${ }^{153}$. Melting studies with mixtures of E10 and E20 with T30 were first performed to validate the predicted hybridization behaviour. Melting temperatures were approximately $30^{\circ} \mathrm{C}$ for $\mathrm{E} 10$ and $\mathrm{T} 30$, and $55^{\circ} \mathrm{C}$ for $\mathrm{E} 20$ and $\mathrm{T} 30$, in close agreement with calculated estimates $^{102}$.

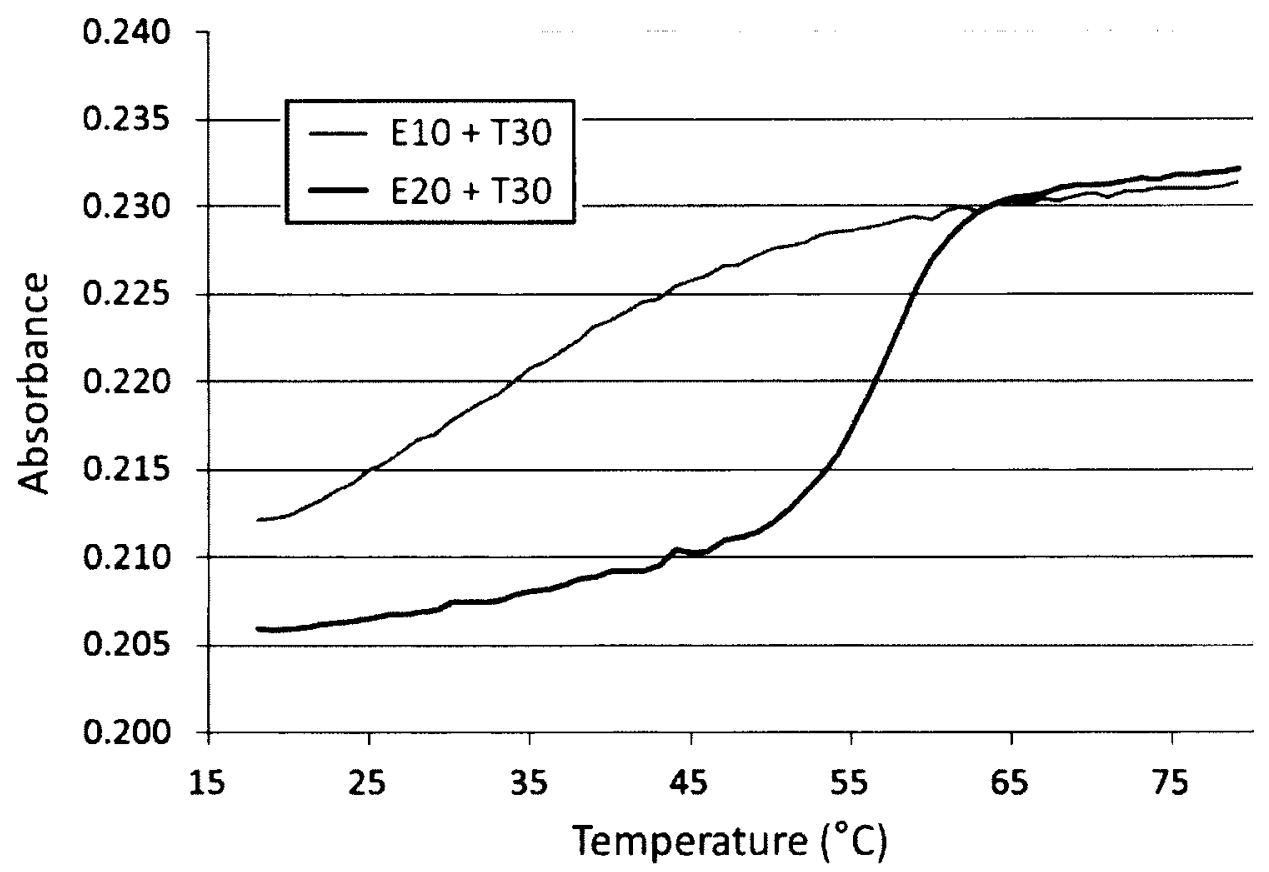

Figure 3-10: Melting study for mixtures of E10+T30 and E20+T30. 
Having confirmed that the desired template structures were formed, conjugation reactions were attempted with DTPA-DA. Mixtures of E10 and T30, and E20 and T30 were heated to $70^{\circ} \mathrm{C}$, cooled to room temperature, and combined with DTPA-DA in either aqueous conjugation buffer or $95 \% \mathrm{~N}, \mathrm{~N}-\mathrm{DMF}$. Supernatants of the reaction mixtures were removed and products isolated by ethanol precipitation were analyzed by PAGE.

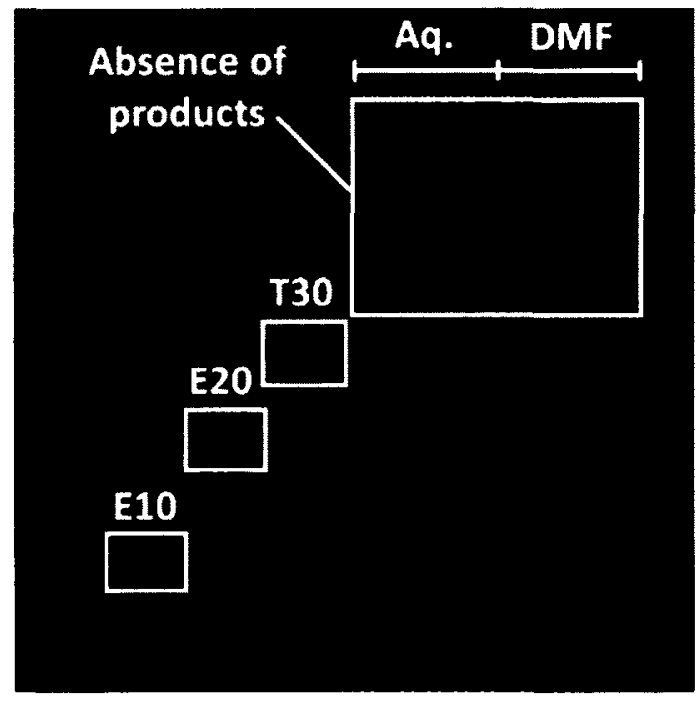

Figure 3-11: Unsuccessful DNA-templated DTPA-DA conjugation reaction. Leftmost lanes contain E10, E20, and E30 markers. Lanes to the right contain oligonucleotides isolated from reaction product supernatants.

After several unsuccessful attempts to produce E10-T30 or E20-T30 conjugates with DTPA-DA, such as that shown in Figure 3-11, disuccinimidyl suberate (DSS) was obtained for testing as an alternative linking reagent. The conjugations reactions were repeated in the same manner. However, instead of removing supernatants from the reaction mixture, the entire sample was concentrated and analyzed by PAGE. Although 
the appearance of the gel in Figure 3-12 was unsightly, most likely induced by incomplete evaporation of $\mathrm{N}, \mathrm{N}-\mathrm{DMF}$ from the samples, there were indeed higher molecular weight conjugation products observed. The bands labeled TS1-TS4 were excised from the gel and the eluted products were analyzed by ESI-MS. The deconvoluted mass spectra are presented in Figure 3-13.

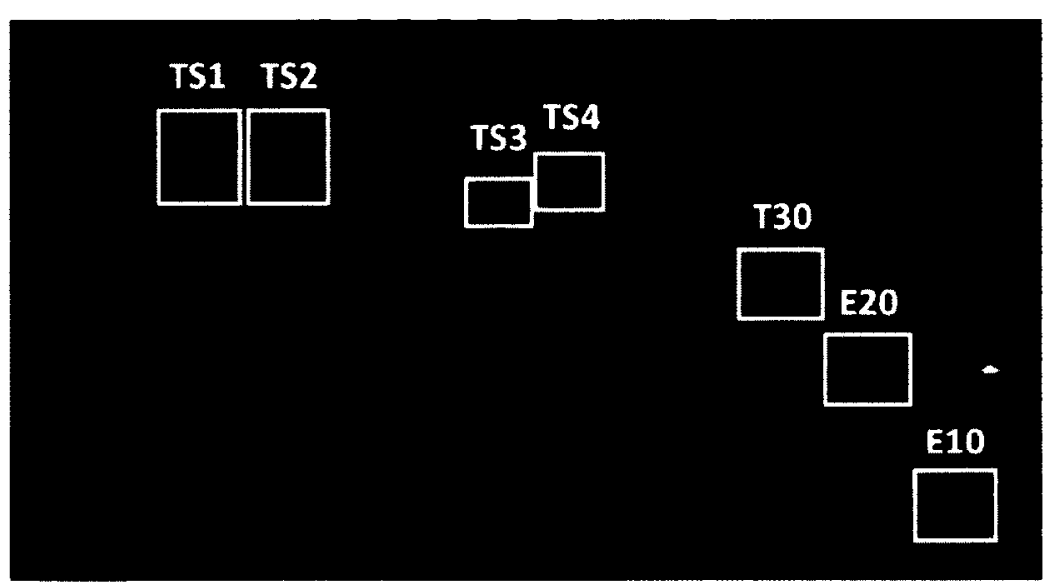

Figure 3-12: Direct PAGE characterization of DNA-templated DTPA-DA and DSS conjugation reaction mixtures. 


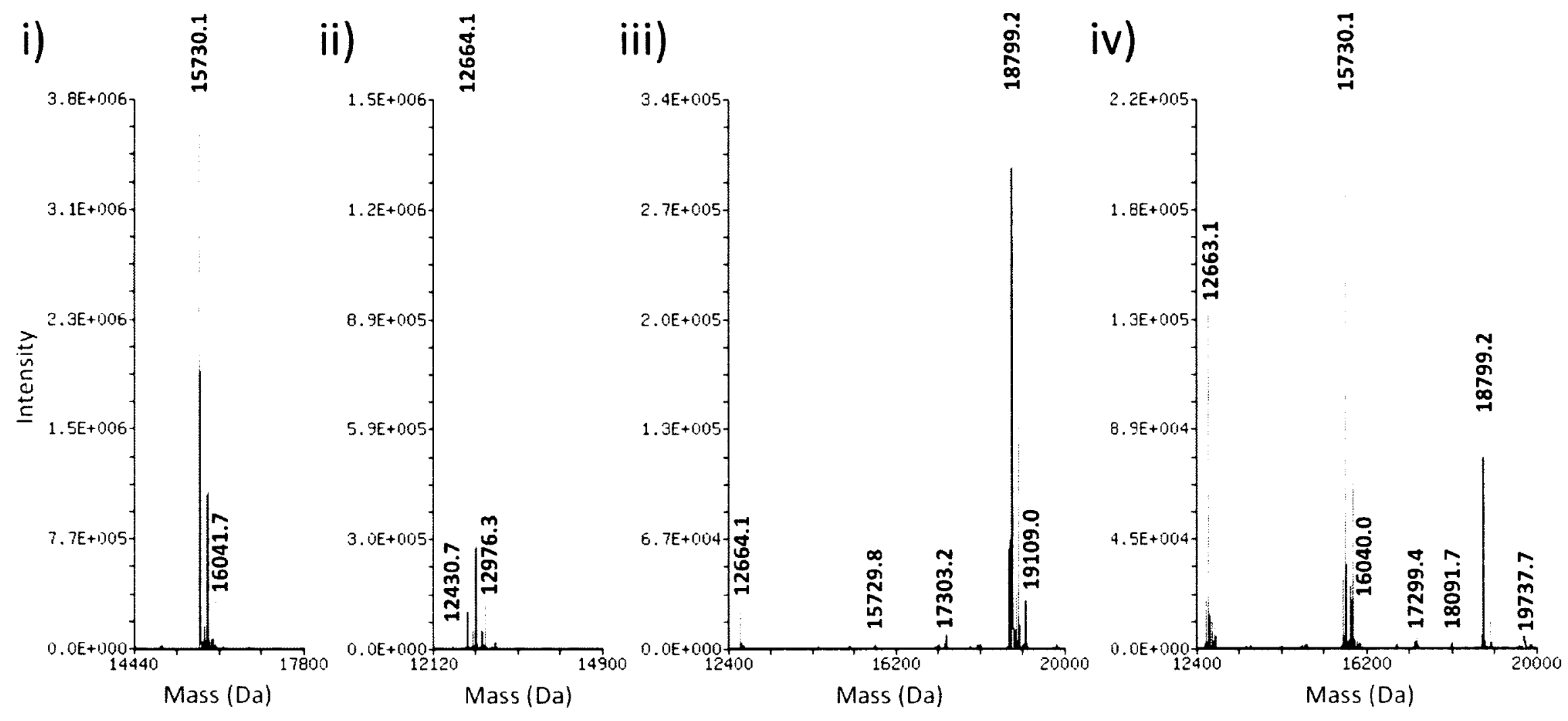

Figure 3-13: Deconvoluted ESI mass spectra of suspected conjugates isolated by PAGE, (i) TS1: E20-DSS-T30, (ii) TS2: E10DTPA-T30, (iii) TS3: E10-DSS-T30, (iv) TS4: E20-DSS-T30. 
None of the major peaks in the mass spectra of excised gel bands TS1-TS4 corresponded to expected reaction products. However, the desired product was identified as a minor peak for TS2 - the E10-DTPA-T30 conjugate at 12920Da. This was the most encouraging observation in this preliminary work with DNA-templated synthesis.

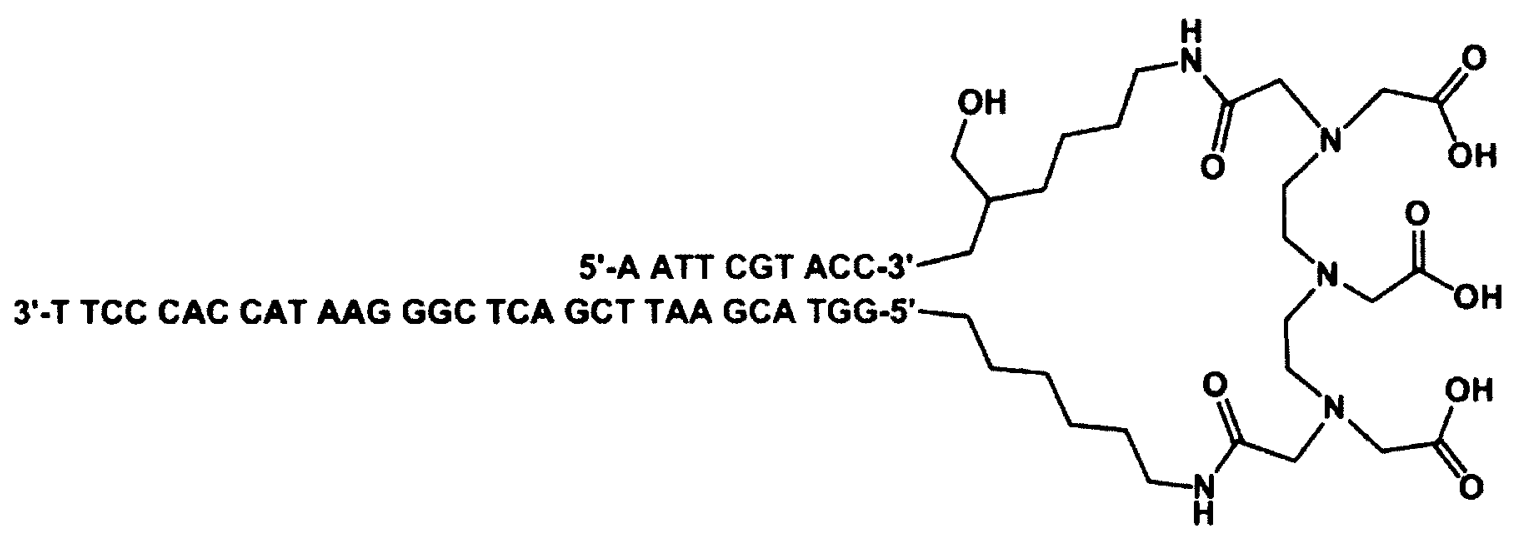

Figure 3-14: Structure of the E10-DTPA-T30 conjugate produced by DNAtemplated synthesis with DTPA-DA.

The major peaks at $15730 \mathrm{Da}$ for TS1 and TS4, and a minor peak at $12664 \mathrm{Da}$ for TS3 would correspond to the expected masses for Lossen reactions that would form $\beta$ alanine linkages between oligonucleotides rather than the expected diamide from the DSS reagent ${ }^{162,163}$. However, the presence of $12664 \mathrm{Da}$ as a major peak for TS2 contradicts this suggestion, as it would represent a Lossen reaction product where no hydroxamic acid derivative was present. Alternatively, apparent deformation of the gel presented as Figure 3-12 may have made it possible that additional products may have migrated into the TS2 lane and were inadvertently included. E10-T30 and E20-T30 Lossen products at $12664 \mathrm{Da}$ and 15730Da respectively appear in both TS3 and TS4, serving as further evidence for cross-contamination. 
Overall, from the masses observed in Figure 3-13, it would seem that the linear template structure employed here seemed more effective at accelerating Lossen reaction linkage products than the desired diamide in the case of DSS. To improve the outcome of DTPA-DA conjugation reactions, new conjugation buffers could be explored. For example, Rozenman et al reported higher yields for DNA-templated amine acylations using a much higher ionic strength conjugation buffer than that employed in the present work $^{153}$.
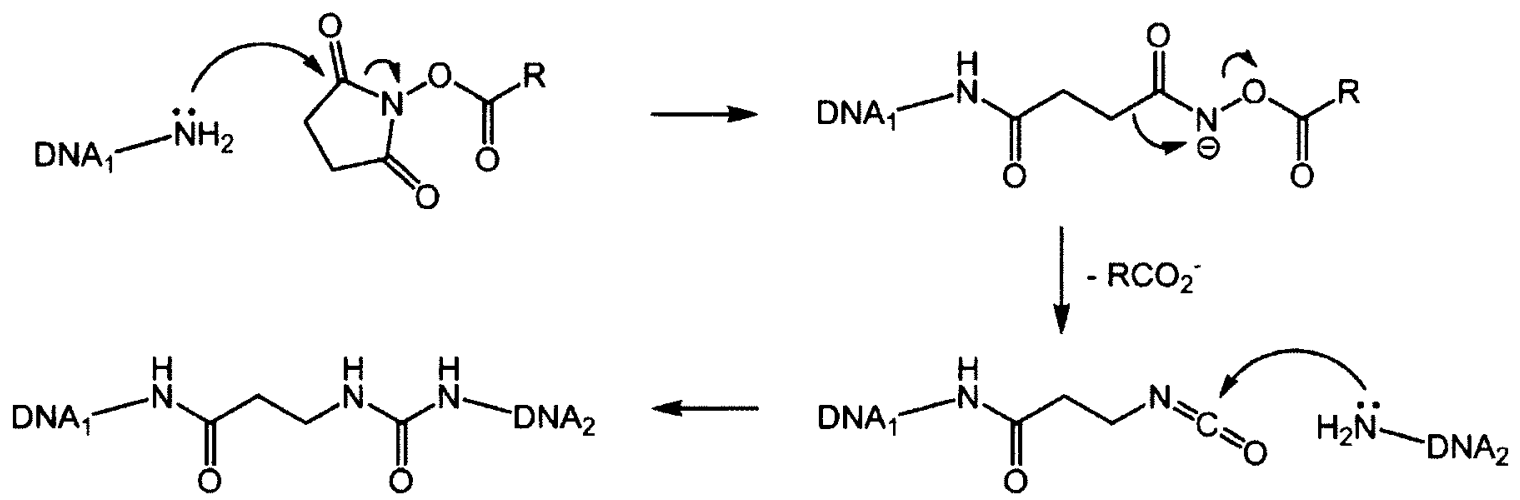

Figure 3-15: Proposed Lossen reaction leading to the formation of interstrand $\beta$ alanine linkages. (adapted from Zalipsky 1998) ${ }^{164}$

While there are several avenues for improvement, most notably with sample cleanup before PAGE analysis, the appearance of the desired product in the mass spectrum of TS2 was encouraging. The linear DNA-template structure from Figure 3-3 i) was employed here for its comparative simplicity. However, more complex structures such as the $\Omega$ architecture shown in Figure 3-3 ii) have resulted in higher yields for many DNA-templated reactions ${ }^{153,154}$. The inclusion of an aptamer sequence in the loop next to the adjacent amino-modifiers as part of the $\Omega$ architecture could also be useful if found to improve dissociation of the template structure for target binding after conjugation. 


\subsection{Conclusions}

Two alterations to the synthesis of aptamer-DTPA $\left(\mathrm{Gd}^{3+}\right)$ conjugates were introduced which significantly improved yields of di-substituted conjugates. The currently developed protocols were capable of producing di-substituted conjugate in a 1:3 ratio with mono-substituted conjugates in the best instances, a significant improvement from their original status as little more than inadvertent byproducts produced at a $\sim 1: 100$ ratio. With the high yield production of mono-conjugates having already been optimized, magnetic characterization was also commenced. At clinically used magnetic field strengths, a relaxivity improvement of about $35 \%$ was measured for aptamerDTPA $\left(\mathrm{Gd}^{3+}\right)$ conjugates in comparison to DTPA $\left(\mathrm{Gd}^{3+}\right)$ alone. While non-target proteins gave relaxivities more comparable to the conjugate alone, target binding doubled the relaxivity improvement to about $70 \%$, a very promising result for the future application of these conjugates as active targeting MRI-CAs. This was corroborated by NMR characterization at higher fields, although the relaxivity improvements were less pronounced. Finally, a DNA-templated synthesis approach to the production of disubstituted conjugates was explored. Oligonucleotides with amino-modifications held in close proximity by a linear template structure were successfully conjugated by DSS, albeit with an undesired linkage produced by an unintended side reaction. The desired conjugation product with DTPA-DA was observed for the shorter of two template constructs, although further work will be required to increase yield. 
Chapter 4 - Conclusion 


\subsection{Thesis Conclusions}

As a contribution to the analytical application of aptamer technology, an electrocatalytic enhancement mechanism inspired by other works in dsDNA mismatch detection was evaluated for compatibility with an established thrombin aptasensor. Protocols were developed for the synthesis and purification the requisite dually modified aptamer, and the preparation of aptamer-modified gold electrode surfaces. After selecting an appropriate buffer and $\mathrm{Fe}(\mathrm{CN})_{6}{ }^{3-}$ substrate concentration, electrocatalytic enhancement was found to improve the sensitivity of the aptasensor $\sim 7$-fold.

The synthesis of aptamer-gadolinium conjugates, proposed as future MRI contrast agents with active-targeting capabilities, was also considerably improved in the present work. While originally produced in a $\sim 1: 100$ ratio with mono-conjugates, the yield of disubstituted conjugates was improved to $1: 3$ in the best instances. Concurrently, magnetic characterization of mono-substituted aptamer-gadolinium conjugates demonstrated a $\sim 70 \%$ increase upon target binding at clinical field strengths. Finally, a DNA-templated synthesis architecture was adopted for the production of asymmetric di-substituted conjugates, which could offer several therapeutic advantages. While further work to increase yield will be required, an asymmetric DTPA di-conjugate based on a 10nt linear architecture was successfully prepared.

Thus, contributions to the analytical and therapeutic applications of aptamer technology have been made by substantially improving both the performance of an electrochemical aptasensor and the synthesis strategies for aptamer-based MRI contrast agents. 


\section{References}

(1) Gold, L.; Ayers, D.; Bertino, J.; Bock, C.; Bock, A.; Brody, E. N.; Carter, J.; Dalby, A. B.; Eaton, B. E.; Fitzwater, T.; Flather, D.; Forbes, A.; Foreman, T.; Fowler, C.; Gawande, B.; Goss, M.; Gunn, M.; Gupta, S.; Halladay, D.; Heil, J.; Heilig, J.; Hicke, B.; Husar, G.; Janjic, N.; Jarvis, T.; Jennings, S.; Katilius, E.; Keeney, T. R.; Kim, N.; Koch, T. H.; Kraemer, S.; Kroiss, L.; Le, N.; Levine, D.; Lindsey, W.; Lollo, B.; Mayfield, W.; Mehan, M.; Mehler, R.; Nelson, S. K.; Nelson, M.; Nieuwlandt, D.; Nikrad, M.; Ochsner, U.; Ostroff, R. M.; Otis, M.; Parker, T.; Pietrasiewicz, S.; Resnicow, D. I.; Rohloff, J.; Sanders, G.; Sattin, S.; Schneider, D.; Singer, B.; Stanton, M.; Sterkel, A.; Stewart, A.; Stratford, S.; Vaught, J. D.; Vrkljan, M.; Walker, J. J.; Watrobka, M.; Waugh, S.; Weiss, A.; Wilcox, S. K.; Wolfson, A.; Wolk, S. K.; Zhang, C.; Zichi, D. PLoS ONE 2010, 5, e1 5004.

(2) Famulok, M.; Hartig, J. S.; Mayer, G. Chem. Rev. 2007, 107, 3715-3743.

(3) Michaud, M.; Jourdan, E.; Ravelet, C.; Villet, A.; Ravel, A.; Grosset, C.; Peyrin, E. Anal. Chem. 2004, 76, 1015-1020.

(4) Tuerk, C.; Gold, L. Science 1990, 249, 505-510.

(5) Ellington, A. D.; Szostak, J. W. Nature 1990, 346, 818-822.

(6) Robertson, D. L.; Joyce, G. F. Nature 1990, 344, 467-468.

(7) Luzi, E.; Minunni, M.; Tombelli, S.; Mascini, M. Trends in Anal. Chem. 2003, 22, 810-818.

(8) Kupakuwana, G. V.; Crill,James E.,,II; McPike, M. P.; Borer, P. N. PLoS ONE 2011, 6, e19395.

(9) Proske, D.; Blank, M.; Buhmann, R.; Resch, A. Appl. Microbiol. Biotechnol. 2005, $69,367-374$.

(10) Monchaud, D.; Allain, C.; Bertrand, H.; Smargiasso, N.; Rosu, F.; Gabelica, V.; De Cian, A.; Mergny, J.; Teulade-Fichou, M. Biochimie 2008, 90, 1207-1223.

(11) Zykovich, A.; Korf, I.; Segal, D. J. Nucleic Acids Res. 2009, 37, e151-el51.

(12) Hermann, T.; Patel, D. J. Science 2000, 287, 820-825.

(13) Geiger, A.; Burgstaller, P.; von, d. E.; Roeder, A.; Famulok, M. Nucleic Acids Res. 1996, 24, 1029-1036.

(14) Liu, J.; Cao, Z.; Lu, Y. Chem. Rev. 2009, 109, 1948-1998. 
(15) Ruigrok, V. J. B.; Levisson, M.; Eppink, M. H. M.; Smidt, H.; van, d. O. Biochem. J. 2011, 436, 1-13.

(16) Famulok, M. J. Med. Chem. 2009, 52, 6951-6957.

(17) Jayasena, S. D. Clin. Chem. 1999, 45, 1628-1650.

(18) Keefe, A. D.; Pai, S.; Ellington, A. Nat. Rev. Drug Discov. 2010, 9, 537-550.

(19) Nimjee, S. M.; Rusconi, C. P.; Sullenger, B. A. Annu. Rev. Med. 2005, 56, 555-583.

(20) Miranda-Castro, R.; de-los-Santos-Alvarez, N.; Lobo-Castanon, M. J.; MirandaOrdieres, A.; Tunon-Blanco, P. Electroanalysis 2009, 21, 2077-2090.

(21) Sefah, K.; Shangguan, D.; Xiong, X.; O'Donoghue, M.,B.; Tan, W. Nat. Protocols 2010, 5, 1169-1185.

(22) Murphy, M. B.; Fuller, S. T.; Richardson, P. M.; Doyle, S. A. Nucleic Acids Res. 2003, 31, e110-e110.

(23) Hoshino, Y.; Koide, H.; Urakami, T.; Kanazawa, H.; Kodama, T.; Oku, N.; Shea, K. J. J. Am. Chem. Soc. 2010, 132, 6644-6645.

(24) Ulyanova, Y. V.; Blackwell, A. E.; Minteer, S. D. Analyst 2006, 131, 257-261.

(25) Evtugyn, G.; Porfireva, A.; Ivanov, A.; Konovalova, O.; Hianik, T. Electroanalysis 2009, 21, 1272-1277.

(26) Vlatakis, G.; Andersson, L. I.; Muller, R.; Mosbach, K. Nature 1993, 361, 645-647.

(27) Warsinke, A.; Nagel, B. Anal. Lett. 2006, 39, 2507-2556.

(28) Puoci, F.; Cirillo, G.; Curcio, M.; Parisi, O. I.; Iemma, F.; Picci, N. Expert Opin. Drug Deliv. 2011, 8, 1379-1393.

(29) Hamula, C. L. A.; Guthrie, J. W.; Zhang, H.; Li, X.; Le, X. C. Trends Anal. Chem. 2006, 25, 681-691.

(30) Tombelli, S.; Minunni, M.; Mascini, M. Biomol. Eng. 2007, 24, 191-200.

(31) Sánchez, J.; Baldrich, E.; Radi, A.; Dondapati, S.; Sánchez, P.; Katakis, I.; $\mathrm{O} \square$ Sullivan, C.Electroanalysis 2006, 18, 1957-1962.

(32) Lubin, A. A.; Plaxco, K. W. Acc. Chem. Res. 2010, 43, 496-505. 
(33) Cho, E. J.; Lee, J.; Ellington, A. D. Annual Review of Analytical Chemistry 2009, 2, 241-264.

(34) Li, D.; Song, S.; Fan, C. Acc. Chem. Res. 2010, 43, 631-641.

(35) Xiao, Y.; Piorek, B. D.; Plaxco, K. W.; Heeger, A. J. J. Am. Chem. Soc. 2005, 127, 17990-17991.

(36) Jager, R. D.; Mieler, W. F.; Miller, J. W. N. Engl. J. Med. 2008, 358, 2606-2617.

(37) Hicke, B.; Stephens, A. W.; Gould, T.; Chang, Y. F.; Lynott, C. K.; Heil, J.; Borkowski, S.; Hilger, C. S.; Cook, G.; Warren, S.; Schmidt, P. G. J. Nucl. Med. 2006, 47, 668-678.

(38) Bock, L. C.; Griffin, L. C.; Latham, J. A.; Vermaas, E. H.; Toole, J. J. Nature 1992, $355,564-566$.

(39) Russo Krauss, I.; Merlino, A.; Giancola, C.; Randazzo, A.; Mazzarella, L.; Sica, F. Nucleic Acids Res. 2011, 39, 7858-7867.

(40) Macaya, R. F.; Schultze, P.; Smith, F. W.; Roe, J. A.; Feigon, J. Proceedings of the National Academy of Sciences 1993, 90, 3745-3749.

(41) Macaya, R. F.; Waldron, J. A.; Beutel, B. A.; Gao, H.; Joesten, M. E.; Yang, M.; Patel, R.; Bertelsen, A. H.; Cook, A. F. Biochemistry (N. Y.) 1995, 34, 4478-4492.

(42) Tasset, D. M.; Kubik, M. F.; Steiner, W. J. Mol. Biol. 1997, 272, 688-698.

(43) Blackburn, G. M.; Gait, M. J.; Loakes, D.; Williams, D. M., Eds.; In Nucleic acids in Chemistry and Biology; RSC Publishing: Cambridge, UK, 2006; Vol. 3rd Edition, pp 470.

(44) Watson, J. D.; Crick, F. H. C. Nature 1953, 171, 737-738.

(45) Beaucage, S. L.; Iyer, R. P. Tetrahedron 1992, 48, 2223-2311.

(46) Beaucage, S. L.; Caruthers, M. H. Tetrahedron Lett. 1981, 22, 1859-1862.

(47) Beaucage, S. L.; Iyer, R. P. Tetrahedron 1993, 49, 1925-1963.

(48) Gilar, M.; Bouvier, E. S. P. Journal of Chromatography A 2000, 890, 167-177.

(49) Pon, R. T.; Usman, N.; Damha, M. J.; Ogilvie, K. K. Nucleic Acids Res. 1986, 14, 6453-6470.

(50) Chrambach, A.; Rodbard, D. Science 1971, 172, 440-451. 
(51) Cornell, B. A.; Braach-Maksvytis, V.; King, L. G.; Osman, P. D. J.; Raguse, B.; Wieczorek, L.; Pace, R. J. Nature 1997, 387, 580-583.

(52) Radi, A. International Journal of Electrochemistry 2011, 2011, 1-17.

(53) Pravda, M. Analytical Methods Electrochemical Analysis. In Encyclopedia of Dairy Sciences; Fuguay, J. W., Ed.; Academic Press: San Diego, 2011; pp 193-197.

(54) Wang, J. Analytical Electrochemistry, 2nd ed.; Wiley-VCH: New York, 2000.

(55) Clark, L. C.; Lyons, C. Ann. N. Y. Acad. Sci. 1962, 102, 29-45.

(56) Sassolas, A.; Leca-Bouvier, B.; Blum, L. J. Chem. Rev. 2008, 108, 109-139.

(57) O'Sullivan, C. Analytical and Bioanalytical Chemistry 2002, 372, 44-48.

(58) Hianik, T.; Wang, J. Electroanalysis 2009, 21, 1223-1235.

(59) Chang, H.; Tang, L.; Wang, Y.; Jiang, J.; Li, J. Anal. Chem. 2010, 82, 2341-2346.

(60) Zhu, Z.; Schmidt, T.; Mahrous, M.; Guieu, V.; Perrier, S.; Ravelet, C.; Peyrin, E. Anal. Chim. Acta 2011, 707, 191-196.

(61) Zhang, X.; Yadavalli, V. K. Biosensors and Bioelectronics 2011, 26, 3142-3147.

(62) Tombelli, S.; Minunni, M.; Luzi, E.; Mascini, M. Bioelectrochemistry 2005, 67, 135141.

(63) Lau, K. Sensors | Overview. In Encyclopedia of Analytical Science; Worsfold, P., Townshend, A., Poole, C., Eds.; Elsevier: Oxford, 2005; pp 224-231.

(64) Franklin, R. K.; Martin, S. M.; Strong, T. D.; Brown, R. B. Chemical Sensors. In Comprehensive Microsystems; Zappe, H., Ed.; Elsevier: Oxford, 2008; pp 433-461.

(65) Bard, A. J.; Faulkner, L. R. Electrochemical Methods: Fundamentals and Applications; Wiley: New York, 2001.

(66) Sawyer, D. T.; Sobkowiak, A.; Roberts, J. L. Electrochemistry for chemists; Wiley: New York, 1995.

(67) Bontempelli, G.; Toniolo, R. Measurement Methods | Electrochemical: Linear Sweep and Cyclic Voltammetry. In Encyclopedia of Electrochemical Power Sources; Garche, J., Ed.; Elsevier: Amsterdam, 2009; pp 643-654. 
(68) Bond, A. M. Electrochemistry: General Introduction. In Comprehensive Coordination Chemistry II; McCleverty, J. A., Meyer, T. J., Eds.; Pergamon: Oxford, 2003; pp 197-222.

(69) Forster, R. J.; Walsh, D. A. Voltammetry | Overview. In Encyclopedia of Analytical Science; Worsfold, P., Townshend, A., Poole, C., Eds.; Elsevier: Oxford, 2005; pp 181-188.

(70) Kounaves, S. P.; O'Dea, J. J.; Chandresekhar, P.; Osteryoung, J. Anal. Chem. 1987, $59,386-389$.

(71) O'Dea, J. J.; Osteryoung, J.; Osteryoung, R. A. Anal. Chem. 1981, 53, 695-701.

(72) White, R. J.; Plaxco, K. W. Anal. Chem. 2010, 82, 73-76.

(73) Rowe, A. A.; Bonham, A. J.; White, R. J.; Zimmer, M. P.; Yadgar, R. J.; Hobza, T. M.; Honea, J. W.; Ben-Yaacov, I.; Plaxco, K. W. PLoS ONE 2011, 6, e23783.

(74) Fan, C.; Plaxco, K. W.; Heeger, A. J. Proceedings of the National Academy of Sciences 2003, 100, 9134-9137.

(75) Palacek, E. Nature 1960, 188, 656-657.

(76) Millan, K. M.; Mikkelsen, S. R. Anal. Chem. 1993, 65, 2317-2323.

(77) Lucarelli, F.; Tombelli, S.; Minunni, M.; Marrazza, G.; Mascini, M. Anal. Chim. Acta 2008, 609, 139-159.

(78) Love, J. C.; Estroff, L. A.; Kriebel, J. K.; Nuzzo, R. G.; Whitesides, G. M. Chem. Rev. 2005, 105, 1103-1170.

(79) Ricci, F.; Zari, N.; Caprio, F.; Recine, S.; Amine, A.; Moscone, D.; Palleschi, G.; Plaxco, K. W. Bioelectrochemistry 2009, 76, 208-213.

(80) Bagotsky, V. S. Fundamentals of Electrochemistry; The Electrochemical Society Series; Wiley: New Jersey, 2006.

(81) Boon, E. M.; Barton, J. K.; Bhagat, V.; Nersissian, M.; Wang, W.; Hill, M. G. Langmuir 2003, 19, 9255-9259.

(82) Kelley, S. O.; Boon, E. M.; Barton, J. K.; Jackson, N. M.; Hill, M. G. Nucleic Acids Res. 1999, 27, 4830-4837.

(83) Farjami, E.; Clima, L.; Gothelf, K. V.; Ferapontova, E. E. Analyst 2010, 135, $1443-$ 1448. 
(84) Kelley, S. O.; Barton, J. K.; Jackson, N. M.; Hill, M. G. Bioconjugate Chem. 1997, 8, 31-37.

(85) Boon, E. M. Electrochemical Sensors Based on DNA-Mediated Charge Transport Chemistry, California Institute of Technology, Pasadena, CA, 2002.

(86) Boon, E. M.; Salas, J. E.; Barton, J. K. Nat. Biotech. 2002, 20, 282-286.

(87) Xiao, Y.; Lubin, A. A.; Heeger, A. J.; Plaxco, K. W. Angew. Chem. Int. Ed. 2005, $44,5456-5459$.

(88) Sadik, O. A.; Aluoch, A. O.; Zhou, A. Biosensors and Bioelectronics 2009, 24, $2749-2765$.

(89) Willner, I.; Zayats, M. Angew. Chem. Int. Ed. 2007, 46, 6408-6418.

(90) Rodriguez, M. C.; Kawde, A.; Wang, J. Chem. Commun. 2005, 4267-4269.

(91) Radi, A.; Acero Sanchez, J. L.; Baldrich, E.; O'Sullivan, C. K. Anal. Chem. 2005, $77,6320-6323$.

(92) Sassolas, A.; Blum, L. J.; Leca-Bouvier, B. D. Electroanalysis 2009, 21, 1237-1250.

(93) Polsky, R.; Gill, R.; Kaganovsky, L.; Willner, I. Anal. Chem. 2006, 78, 2268-2271.

(94) Riddles, P. W.; Blakeley, R. L.; Zerner, B. Anal. Biochem. 1979, 94, 75-81.

(95) Ge, B.; Huang, Y.; Sen, D.; Yu, H. J Electroanal. Chem. 2007, 602, 156-162.

(96) Carpino, L. A.; Han, G. Y. J. Org. Chem. 1972, 37, 3404-3409.

(97) Andrus, A.; Kuimelis, R. G. Analysis and Purification of Synthetic Nucleic Acids Using HPLC. In Current Protocols in Nucleic Acid Chemistry; John Wiley \& Sons, Inc: 2001.

(98) Schubert, F.; Knaf, A.; Moller, U.; Cech, D. Nucleosides \& Nucleotides 1995, 14, 1437-1443.

(99) Lewis, G. N.; Goldschmid, O.; Magel, T. T.; Bigeleisen, J. J. Am. Chem. Soc. 1943, $65,1150-1154$.

(100) Cavaluzzi, M. J.; Borer, P. N. Nucleic Acids Res. 2004, 32, e13-e13.

(101) Cech, N. B.; Enke, C. G. Mass Spectrom. Rev. 2001, 20, 362-387.

(102) Kibbe, W. A. Nucleic Acids Res. 2007, 35, W43-W46. 
(103) Tuite, E.; Kelly, J. M. Biopolymers 1995, 35, 419-433.

(104) Eyer, P.; Worek, F.; Kiderlen, D.; Sinko, G.; Stuglin, A.; Simeon-Rudolf, V.; Reiner, E. Anal. Biochem. 2003, 312, 224-227.

(105) Chen, W.; Zhao, Y.; Seefeldt, T.; Guan, X. J. Pharm. Biomed. Anal. 2008, 48, 1375-1380.

(106) Geisthardt, D.; Kruppa, J. Anal. Biochem. 1987, 160, 184-191.

(107) Barth, H. G.; Jackson, C.; Boyes, B. E. Anal. Chem. 1994, 66, 595R-620R.

(108) Herne, T. M.; Tarlov, M. J. J. Am. Chem. Soc. 1997, 119, 8916-8920.

(109) Ulman, A. Chem. Rev. 1996, 96, 1533-1554.

(110) Davis, F.; Higson, S. P. J. Biosensors and Bioelectronics 2005, 21, 1-20.

(111) Steel, A. B.; Herne, T. M.; Tarlov, M. J. Anal. Chem. 1998, 70, 4670-4677.

(112) Radi, A.; Acero Sanchez, J. L.; Baldrich, E.; O'Sullivan, C. K. J. Am. Chem. Soc. 2006, $128,117-124$.

(113) Radi, A.; O'Sullivan, C. K. Chem. Commun. 2006, 3432-3434.

(114) Zhang, J.; Wang, L.; Pan, D.; Song, S.; Fan, C. Chem. Commun. 2007, 1154-1156.

(115) Huang, S.; Schopf, E.; Chen, Y. Nano Lett. 2007, 7, 3116-3121.

(116) Ceres, D. M.; Barton, J. K. J. Am. Chem. Soc. 2003, 125, 14964-14965.

(117) Widrig, C. A.; Chung, C.; Porter, M. D. Journal of Electroanalytical Chemistry and Interfacial Electrochemistry 1991, 310, 335-359.

(118) Kemmer, G.; Keller, S. Nat. Protocols 2010, 5, 267-281.

(119) Cheng, A. K. H.; Sen, D.; Yu, H. Bioelectrochemistry 2009, 77, 1-12.

(120) White, R. J.; Phares, N.; Lubin, A. A.; Xiao, Y.; Plaxco, K. W. Langmuir 2008, 24, 10513-10518.

(121) Shen, L.; Chen, Z.; Li, Y.; Jing, P.; Xie, S.; He, S.; He, P.; Shao, Y. Chem. Commun. 2007, 2169-2171.

(122) Ferguson, B. S.; Buchsbaum, S. F.; Swensen, J. S.; Hsieh, K.; Lou, X.; Soh, H. T. Anal. Chem. 2009, 81, 6503-6508. 
(123) Floyd, W. C.; Klemm, P. J.; Smiles, D. E.; Kohlgruber, A. C.; Pierre, V. C.; Mynar, J. L.; Frechet, J. M. J.; Raymond, K. N. J. Am. Chem. Soc. 2011, 133, 2390-2393.

(124) Liang, G.; Ronald, J.; Chen, Y.; Ye, D.; Pandit, P.; Ma, M. L.; Rutt, B.; Rao, J. Angew. Chem. Int. Ed. 2011, 50, 6283-6286.

(125) Kadjane, P.; Logothetis, N. K.; Angelovski, G. Tetrahedron Lett. 2011, 52, 16191622.

(126) Kamaly, N.; Miller, A.,D.; Bell, J.,D. Current Topics in Medicinal Chemistry 2010, $10,1158-1183$.

(127) Caravan, P.; Farrar, C. T.; Frullano, L.; Uppal, R. Contrast Media Mol. Imaging 2009, 4, 89-100.

(128) Broome, D. R. Eur. J. Radiol. 2008, 66, 230-234.

(129) Caravan, P.; Ellison, J. J.; McMurry, T. J.; Lauffer, R. B. Chem. Rev. 1999, 99, 2293-2352.

(130) Hermann, P.; Kotek, J.; Kubicek, V.; Lukes, I. Dalton Trans. 2008, 3027-3047.

(131) Hines, J. V.; Ammar, G. M.; Buss, J.; Schmalbrock, P. Bioconjugate Chem. 1999, $10,155-158$.

(132) Vaughan, T.; DelaBarre, L.; Snyder, C.; Tian, J.; Akgun, C.; Shrivastava, D.; Liu, W.; Olson, C.; Adriany, G.; Strupp, J.; Andersen, P.; Gopinath, A.; van de Moortele, P.; Garwood, M.; Ugurbil, K. Magn. Reson. Med. 2006, 56, 1274-1282.

(133) Kribben, A.; Witzke, O.; Hillen, U.; Barkhausen, J.; Daul, A. E.; Erbel, R. J. Am. Coll. Cardiol. 2009, 53, 1621-1628.

(134) Sadowski, E. A.; Bennett, L. K.; Chan, M. R.; Wentland, A. L.; Garrett, A. L.; Garrett, R. W.; Djamali, A. Radiology 2007, 243, 148-157.

(135) Colletti, P. M. Am. J. Roentgenol. 2008, 191, 1150-1153.

(136) Godin, B.; Tasciotti, E.; Liu, X.; Serda, R. E.; Ferrari, M. Acc. Chem. Res. 2011, $44,979-989$.

(137) Lucas, R. L.; Benjamin, M.; Reineke, T. M. Bioconjugate Chem. 2008, 19, 24-27.

(138) De Leon-Rodriguez, L. M.; Lubag, A.; Udugamasooriya, D. G.; Proneth, B.; Brekken, R. A.; Sun, X.; Kodadek, T.; Dean Sherry, A. J. Am. Chem. Soc. 2010, $132,12829-12831$. 
(139) Sukerkar, P. A.; MacRenaris, K. W.; Meade, T. J.; Burdette, J. E. Mol. Pharmaceutics 2011, 8, 1390-1400.

(140) Louie, A. Y.; Huber, M. M.; Ahrens, E. T.; Rothbacher, U.; Moats, R.; Jacobs, R. E.; Fraser, S. E.; Meade, T. J. Nat. Biotech. 2000, 18, 321-325.

(141) Song, Y.; Kohlmeir, E. K.; Meade, T. J. J. Am. Chem. Soc. 2008, 130, 6662-6663.

(142) Frullano, L.; Tejerina, B.; Meade, T. J. Inorg. Chem. 2006, 45, 8489-8491.

(143) Frullano, L.; Zhu, J.; Wang, C.; Wu, C.; Miller, R. H.; Wang, Y. J. Med. Chem. 2011.

(144) Xu, W.; Lu, Y. Chem. Commun. 2011, 47, 4998-5000.

(145) Hong, H.; Goel, S.; Zhang, Y.; Cai, W. Curr. Med. Chem. 2011, 18, 4195-4205.

(146) Beking, M. A. Development of Aptamer-Gadolinium Conjugates as MRI Contrast Agents, Carleton University, Ottawa, ON, 2008.

(147) Hua, A. Adv. Drug Deliv. Rev. 2011, 63, 772-788.

(148) Wang, A. Z.; Bagalkot, V.; Vasilliou, C. C.; Gu, F.; Alexis, F.; Zhang, L.; Shaikh, M.; Yuet, K.; Cima, M.; Langer, R.; Kantoff, P. W.; Bander, N. H.; Jon, S.; Farokhzad, O. C. ChemMedChem 2008, 3, 1311-1315.

(149) Norek, M.; Peters, J. A. Prog. Nucl. Magn. Reson. Spectrosc. 2011, 59, 64-82.

(150) Kleiner, R. E.; Brudno, Y.; Birnbaum, M. E.; Liu, D. R. J. Am. Chem. Soc. 2008, $130,4646-4659$.

(151) Li, X.; Liu, D. R. Angew. Chem. Int. Ed. 2004, 43, 4848-4870.

(152) Sharma, A. K.; Heemstra, J. M. J. Am. Chem. Soc. 2011, 133, 12426-12429.

(153) Rozenman, M. M.; Liu, D. R. ChemBioChem 2006, 7, 253-256.

(154) Gartner, Z. J.; Grubina, R.; Calderone, C. T.; Liu, D. R. Angew. Chem. Int. Ed. 2003, 42, 1370-1375.

(155) Hnatowich, D. J.; Layne, W. W.; Childs, R. L.; Lanteigne, D.; Davis, M. A.; Griffin, T. W.; Doherty, P. W. Science 1983, 220, 613-615.

(156) Laurent, S.; Vander Elst, L.; Henoumont, C.; Muller, R. N. Contrast Media Mol. Imaging 2010, 5, 305-308. 
(157) Vargas-Baca, I.; Mitra, D.; Zulyniak, H. J.; Banerjee, J.; Sleiman, H. F. Angew. Chem. Int. Ed. 2001, 40, 4629-4632.

(158) Sherry, A. D.; Caravan, P.; Lenkinski, R. E. J. Magn. Reson. Imaging 2009, 30, 1240-1248.

(159) Munshi, K.; Dey, A. Mikrochim. Acta 1968, 56, 1059-1065.

(160) Laurent, S.; Botteman, F.; Elst, L.; Muller, R. HCA 2004, 87, 1077-1089.

(161) Koenig, S. H. Journal of Magnetic Resonance 1978, 31, 1-10.

(162) Isidro-Llobet, A.; Just-Baringo, X.; Ewenson, A.; Álvarez, M.; Albericio, F. Biopolymers 2007, 88, 733-737.

(163) Narendra, N.; Chennakrishnareddy, G.; Sureshbabu, V. V. Org. Biomol. Chem. 2009, 7, 3520-3526.

(164) Zalipsky, S. Chem. Commun. 1998, 69-70. 